\title{
Carrying on or giving in : processes of self-control and ego depletion
}

Citation for published version (APA):

Alberts, H. J. E. M. (2007). Carrying on or giving in : processes of self-control and ego depletion. [Doctoral Thesis, Maastricht University]. Datawyse / Universitaire Pers Maastricht. https://doi.org/10.26481/dis.20070621ha

Document status and date:

Published: 01/01/2007

DOI:

10.26481/dis.20070621ha

Document Version:

Publisher's PDF, also known as Version of record

\section{Please check the document version of this publication:}

- A submitted manuscript is the version of the article upon submission and before peer-review. There can be important differences between the submitted version and the official published version of record.

People interested in the research are advised to contact the author for the final version of the publication, or visit the DOI to the publisher's website.

- The final author version and the galley proof are versions of the publication after peer review.

- The final published version features the final layout of the paper including the volume, issue and page numbers.

Link to publication

\footnotetext{
General rights rights.

- You may freely distribute the URL identifying the publication in the public portal. please follow below link for the End User Agreement:

www.umlib.nl/taverne-license

Take down policy

If you believe that this document breaches copyright please contact us at:

repository@maastrichtuniversity.nl

providing details and we will investigate your claim.
}

Copyright and moral rights for the publications made accessible in the public portal are retained by the authors and/or other copyright owners and it is a condition of accessing publications that users recognise and abide by the legal requirements associated with these

- Users may download and print one copy of any publication from the public portal for the purpose of private study or research.

- You may not further distribute the material or use it for any profit-making activity or commercial gain

If the publication is distributed under the terms of Article $25 \mathrm{fa}$ of the Dutch Copyright Act, indicated by the "Taverne" license above, 


\section{CARRYING ON OR GIVING IN Processes of self-control and ego depletion}




\section{Colophon}

Graphic design: Hugo Alberts

Production: Datawyse | Universitaire Pers Maastricht

(C) 2007 Hugo Alberts, Maastricht 2007

ISBN 978-90-5278-636-0

The research presented in this dissertation was supported by a grant of the Netherlands Organization for Scientific Research (NWO, Grant No. 402-01-049).

All rights are reserved. No part of this book may be reproduced or transmitted in any form or by any means, without written permission from the author or, when appropriate, the publisher of the article. 


\section{CARRYING ON OR GIVING IN \\ Processes of self-control and ego depletion}

\section{PROEFSCHRIFT}

ter verkrijging van de graad van doctor aan de Universiteit Maastricht, op gezag van de Rector Magnificus, Prof. Mr. G.P.M.F. Mols, volgens het besluit van het College van Decanen, in het openbaar te verdedigen op donderdag 21 juni 2007 om 16.00 uur

door

Hugo Jules Emile Maria Alberts 


\section{Promotores}

Prof. dr. N.K. de Vries

Prof. dr. H. Merckelbach

\section{Copromotor}

Dr. C. Martijn

\section{Beoordelingscommissie}

Prof. dr. A. Jansen (voorzitter)

Prof. dr. D. de Ridder (Universiteit Utrecht)

Dr. G.P.J. Keijsers (Radboud Universiteit Nijmegen)

Prof. dr. G. Kok

Prof. dr. J. Vlaeyen (Katholieke Universiteit Leuven) 


\section{CONTENTS}

Chapter 1

Chapter 2

Chapter 3

Chapter 4

Chapter 5

Chapter 6

Chapter 7
General Introduction

7

Self-control related expectancies and 19 self-control abilities

The role of automatic processes in 29 overcoming ego depletion

Attentional focus and self-control 53 performance

Differential effects of self-focused attention on self-control performance

Understanding impulsive crime:

The role of state self-control

General Discussion

101

Summary

Samenvatting

References

Dankwoord

Curriculum Vitae 



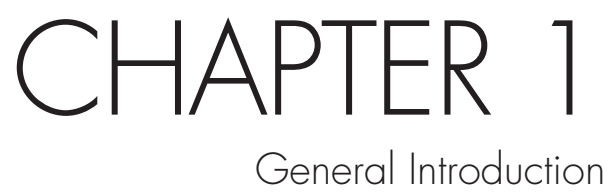


Since the earliest dawn of civilization, self-control has been recognized as one of the highest virtues. Greek philosophers like Plato and Aristotle considered self-control of equal value to courage, wisdom, and justice. Mastery over one's desires, faults, and lusts was argued to be indispensable in the human pursuit of happiness. Lucius Seneca, a first century Roman philosopher captured the essence of this rationale by stating that "Most powerful is he who has himself in his own power." Even in today's modern society, the interest in self-control as a central human capacity seems unaltered. Self-control is associated with a diversity of both social and economic developments and has often been referred to as the "cornerstone of civilization". This analogy seems justified: many of the problems that confront our society can be explained in terms of self-control failure. Widespread problems like obesitas, drug addiction and violence reflect people's inability to control their food intake, cravings and aggressive impulses. Unfortunately, these problems do not only emerge on an individual level, but go beyond the individual as well by representing significant disruptions on a societal level. For instance, self-control failure in the form of unsafe sexual intercourse is not only hazardous for the individuals involved but, in a larger context, also contributes to the spread of sexual transmitted diseases. Moreover, deficient self-control has been identified as one of the most important factors contributing to the pervasive problem of criminality (Gottfredson \& Hirschi, 1990). Research in the field of criminology has found that individuals who possess low self-control abilities have a higher likelihood of criminal activity than those with high self-control abilities (e.g., Burton et al., 1998; LaGrange \& Silverman, 1999; Vazsonyi et al. 2001). Not surprisingly, given these negative consequences of low self-control, good self-control abilities have been found to contribute to overall quality of life. Recent research findings support what was preached by the ancient philosophers ages ago: self-control and happiness are strongly related (Tangney, Baumeister, \& Boone, 2004). As compared to people low in selfcontrol, this research found that people who possess relatively good self-control skills not only showed fewer impulse control problems, but also higher self-esteem, better relationships, better interpersonal skills and more optimal emotional responses (see also Lu \& Shih, 1997; Larson, 1989; Finkel \& Campbell, 2001). In addition, a longitudinal study by Mischel, Shoda and Peake (1988) considered the impact of self-control abilities from a long-term perspective. It was found that 5 and 6 year old children, who were better able to resist immediate temptations and choose delayed gratifications, grew into more successful and well-adjusted adolescents, as rated by their parents a decade later. More specifically, these children were rated as more academically and so- 
cially competent, verbally fluent, rational, attentive, planful, and able to deal well with frustration and stress. Together, these findings illustrate the value of self-control and stress the importance of gaining more insight in the ability to control ourselves. The purpose of the present dissertation is to gain a better understanding of the processes that underlie self-control performance. To do so, the emphasis will be on the identification of distinct factors that influence (effective) self-control. Uncovering these factors will not only reveal more of the underlying processes of self-control, but will also provide insight in possible ways to improve self-control performance.

\section{Self-control defined}

Self-control can be regarded as an ability that underlies a wide range of human behaviours. As a result, it has become a common theme in many of today's different fields of research. Over the past thirty years, a large but diverse body of research covering different aspects and viewpoints of self-control has been presented in the literature (for overviews see Boekaerts, Pintrich, \& Zeidner, 2000). As an inevitable consequence of this diversity, the present dissertation was compelled to address a specific perspective on self-control. In the current work, the focus will be on a social cognitive perspective on self-control. From this point of view, self-control can be defined as the overriding or inhibition of urges, behaviours or desires (Baumeister, Heatherton, \& Tice, 1994; Shallice \& Burgess, 1993). For instance, a dieter must control the desire to eat from a pie in order to prevent him or herself from breaking the diet. Controlling your weight or giving up smoking implies that you have to say 'no' to yourself instead of giving in to craving or appetite.

Although self-control is often used as a synonym for self-regulation, in the present dissertation it will be used in a more narrow sense. Self-regulation refers to a broad, regulatory cycle of comparing the self against a relevant standard, operating on the self to reduce a possible discrepancy, testing again and continuing the process until a test reveals that the standard has been reached, whereupon the person exits the circle (test, operate, test, exit (TOTE), cf. Carver \& Scheier, 1981). According to Baumeister and Exline (2000), self-control refers to the "operate" phase, in which the self alters itself in order to move closer to the desired standard. In practice, this often means the overruling of one incipient pattern of response and replacing it with another (Baumeister, 2002). Thus, the individual suppresses an unwanted thought and tries to think of something else, or he/she ignores the elevator and takes the stairs instead. This active 
control process serves to reduce the risk that any thought, feeling or behaviour that is incompatible with the desired standard could take over. It is exactly this phase of active self-control that entails the main focus of the present dissertation.

As is the case with many psychological constructs, self-control can be understood as a relatively stable, enduring personality trait, as well as a behaviour that is susceptible to momentary changes. Thus, self-control is the outcome of both personality and situational influences. Trait self-control refers to an individual's general self-control capabilities. Trait self-control develops during lifetime and can be defined as a relatively stable characteristic of the person possessing it (Tangney, Baumeister \& Boone, 2004). State self-control, on the other hand, concerns the intra-individual variability of selfcontrol over time or situations (Hong, 1998; Nesselroade \& Bartsch, 1977). State selfcontrol is relatively changeable and can be influenced by situational factors. Trait selfcontrol and state self-control are strongly related, since an individual with high trait self-control is more likely to successfully exert self-control in a given situation (Hong, 1998). In the present dissertation, both state and trait self-control will be addressed.

\section{The strength model}

According to the so-called strength model (also known as the ego-strength model or resource model), the capacity to control ourselves is defined by our own restrictions (Muraven, Tice, \& Baumeister, 1998; Muraven \& Baumeister, 2000). These restrictions are a direct consequence of self-control drawing on a fixed and limited resource, akin to strength or energy. After an act of self-control, the resource becomes temporarily depleted leaving the individual in a state of ego depletion. Therefore, performance on a subsequent self-control act will be impaired because of lack of resources. The strength model ascribes a number of features to the concept of energy resource. First, the resource is non-specific and used for a broad variety of self-control operations such as overriding or inhibiting impulses, regulating emotions, making choices and so on. Second, the resource is limited, that is, the energy required to inhibit the desire to eat a fattening snack is no longer available to suppress a negative emotion. Third, self-control is similar to a muscle. Like a muscle, self-control becomes tired after use and needs rest to recover. In addition, similar to training a muscle, self-control should also become stronger with repeated practice. This strengthening may offer a strategy to counter regulatory failure (Muraven, Baumeister, \& Tice, 1999; Oaten \& Cheng, 2006).

The first empirical demonstration of the assumption that self-control depends on 
available energy comes from Baumeister, Bratslavsky, Muraven and Tice (1998), who reported a series of four experiments on the ego depletion phenomenon. In the most appealing experiment, Baumeister and colleagues invited 3-hour food-deprived participants to a laboratory room that was filled with the aroma of freshly baked chocolate cookies (Experiment 1). Participants were confronted with two displays of food: a stack of fresh chocolate cookies and a bowl of radishes. Next, some participants were instructed to eat only from the radishes and not from the cookies (high self-control), whereas other participants were instructed to eat the cookies (low self-control). In each condition, participants were instructed to eat at least some of the food item they were allowed to eat (some radishes in the radish condition or a cookie in the chocolate condition). This manipulation intended to manipulate participants' self-control; participants in the radish condition were hungry, and then confronted with deliciously smelling food. However, they were only permitted to eat the radishes and not allowed to touch the cookies. After that, all participants took part in a 'problem-solving task' that consisted of a series of geometrical figure-tracing puzzles. What participants did not know was that these puzzles were in fact unsolvable and that the time they spent on this frustrating task served as a measure of their self-control. A group of control participants was not subjected to the food part of the experiment but started directly with the puzzle task. The exertion of self-control, that is, resisting chocolate cookies, was found to undermine subsequent persistence with unsolvable puzzles. Participants who refrained themselves from eating cookies spent relatively less time and made less attempts to solve the puzzles than participants who were allowed to eat chocolate cookies and control participants who were neither hungry or exposed to food. Thus, initial exercise of self-control (refraining from eating attractive food when hungry), hampered participants' behaviour on a second, unrelated self-control task (persisting on a frustrating task).

By now, the phenomenon of ego depletion is a well-researched phenomenon and empirical tests have shown that self-regulatory resources underlie a wide range of behaviour. Examples of findings are that the regulation of thoughts (i.e., suppressing a specific thought), altering one's emotional state (i.e., suppressing or exaggerating happy or sad emotions), and decision making involve self-control and that each of these activities results in impaired performance on a second self-control activity (see for an overview, Schmeichel, \& Baumeister, 2004). For instance, a recent study by Stucke and Baumeister (2006) investigated the relationship between ego depletion and aggressive behaviour. In this study, participants were depleted by resisting the temptation to 
consume tasty food (Experiment 1) or by sitting motionless and expressionless while watching a boring movie (Experiment 2 and 3). Next, participants were asked to complete a so-called creativity task. After completing this task, the experimenter insulted participants by making rude comments on the way they completed the task. In order to measure aggressive responses toward the experimenter, participants were asked to evaluative the experimenter. Participants were aware that their evaluation would influence the academic position of the experimenter. A negative judgement was likely to have negative or even harmful consequences for the experimenter. The results showed that depleted participants gave more negative evaluations and thus responded more aggressively than control participants.

In sum, according to the strength model, self-control relies upon a limited resource of energy. When people control themselves, they use an amount of energy that is unavailable for future self-control demands, resulting in decreased performance. Findings across studies have supported the strength model and have illustrated the negative impact of repeated self-control on a diversity of behaviours.

\section{Underlying processes}

Despite the substantial body of literature illustrating the ego depletion phenomenon, the precise nature of limited strength and the psychological process underlying this limitation remains unclear. So far, most of the studies on ego depletion attempted to identify behaviours and outcome measures that are affected by ego depletion. Although the identification of these different outcome measures can be considered as a valuable contribution to the field, it does not provide more insight in the processes that underlie self-control and ego depletion. The concept of energy is, as Metcalfe and Mischel put it "much in need of a theory that specifies how it comes about and operates and when it goes up and down, and who has it and who lacks it" (1999, p. 16). Stated differently, the strength model seems to lack process and, at this point, the concept of energy may be best compared to a black box. The present dissertation will address this issue so as to gain more insight in the processes that underlie self-control performance and ego depletion. In doing so, a perspective was adopted that approaches self-control from a rather different angle than the earlier described strength model. Whereas the limited energy model is mainly concerned with the limits of our capacity to exert self-control, the research reported in the present dissertation was mainly guided by a focus on the

flexibility and relativity of regulatory capabilities. Instead of considering self-control 
failure as an inevitable consequence of prior consumption, the current work was directed by a different view on self-control in which regulatory limits are considered less static and more susceptible to adjustment. It was argued that by focussing on possible ways to improve self-control and to circumvent ego depletion, the strength model can be extended and room can be created for identification of additional factors and processes that contribute to self-control performance.

Indeed, previous studies have found some evidence for the view that the limits of self-control are less static than suggested by the limited strength model. For instance, Muraven and Slessarava (2003) conducted a series of experiments in which they demonstrated that ego depletion may be compensated by increasing people's motivation to perform well on subsequent self-control task. They showed that initially depleted individuals persevered longer at a subsequent self-control task if they were led to believe that their persistence would be beneficial to others or to themselves. Depleted participants, who were not motivated, lowered their persistence and thus showed ego depletion. Muraven and Slessarava explained the compensatory effect of motivation on ego depletion in terms of incentives that motivate people to use more of their energy resources. If such an incentive is absent, individuals who already used up a part of their resources tend to conserve their leftover energy, which is reflected in impaired selfcontrol performance on a subsequent task. Yet, if breaking into one's reserves seems worthwhile (either for one's own benefit or that of others), the tendency to mobilize costly energy will overrule the tendency to conserve. Thus, Muraven and Slessarava concluded that the natural (not necessarily conscious) tendency of depleted individuals is to conserve their scarce resources, and what is left will only be brought to action in the presence of an important goal. In other words, although the strength model and its muscle analogy may seem powerful and appealing, these findings illustrate that the role of the athlete is put somehow out of action. After all, self-control does not only involve (mental) muscles but also a brain: it is the athlete who decides whether the game is important enough to give it all or to save some for the next. Obviously, determining whether the stake is high enough and acting upon it, is a consideration that requires a brain, and not only muscle.

The flexibility of our self-control abilities was also demonstrated in a study by Richards and Gross (2001). This study illustrated the strategic nature of self-control and showed that construing an emotional situation in a way that decreases its emotional relevance (antecedent-focused regulation), is considerably less cognitively costly than regulating one's emotional reactions during the occurrence of this situation (re- 
sponse-focused regulation). For instance, in one experiment it was found that suppression of emotions while watching photos of heavily injured people required less effort if participants were instructed to watch them with the cool, distant eye of a medical doctor so as to turn away from one's own emotional reactions (fear, disgust). Together, the findings of this study suggest that the cognitive costs of emotion regulation vary according to how emotions are regulated. By using appropriate strategies, self-regulation may be facilitated and less cognitively demanding. Thus, the loss or maintenance of self-control does not only seem to be a matter of strength but also of strategy.

Further evidence for the relativity of limits was provided in a in a study by Martijn, Tenbült, de Vries, Dreezens, and Merckelbach (2002). These authors demonstrated that the perceived boundaries of one's self-controlling abilities are apt to change. In this study, participants watched a highly disgusting video fragment and were asked to suppress their emotions. A control group watched the same video, but received no suppression instructions. Immediately after, half of the participants who suppressed their emotions were informed that "often people think they have to rest after an effortful task. However, scientific investigations prove that this is not the case after emotional effort..." (Martijn et al., 2002; p. 446, Experiment 1). On a subsequent self-control demanding task, participants who received this information outperformed control participants and participants who received no such information. These results suggest that people expect that exercising self-control leads to fatigue and, hence to worse performance on a second self-control demanding task. If such expectancy is challenged, decline in performance is less evident and people may even achieve better.

Taken together, recent findings suggest that depletion of strength is less inevitable and automatic than assumed by the limited strength model. Whereas the strength model stresses the limits of our capability to control ourselves, the studies described above rather stress the flexibility and relativity of these limits. These studies give rise to the idea that the concept of energy is likely to be more complicated and dynamic than initially suggested.

\section{The present dissertation}

As already discussed in the preceding section, the aim of the present dissertation is to gain more insight in the processes that underlie self-control performance. The studies presented in the following chapters address the role of distinct types of psychological processes on self-control performance and the occurrence of ego depletion. 
Previous research on self-control has suggested that self-control performance and the occurrence of the ego depletion phenomenon may be influenced by expectancies or schemata about self-control (Martijn et al., 2002; Mukhopadhyay \& Johar, 2005). The results of these studies imply that not the existence of a limited energy resource per se, but the (naïve) expectancy that self-control is dependent on limited energy may explain self-control failure. In Chapter 2, three studies will be described that address this possible link between self-control related beliefs and self-control performance. More specifically, we tested whether the extent to which people believe that self-control is dependent on limited energy correlates with their own perception of their self-regulatory skills.

Chapter 3 examines the impact of automatic processes on self-control. Previous studies addressing the possible counteraction of ego depletion have relied on various means such as training or explicitly increasing the benefits of enacting self-control. However, these studies relied on explicit manipulations, requiring a certain amount of conscious attention and processing. In chapter 3 , two studies will be described that investigate whether the manifestation of ego depletion can also be reduced or even eliminated by means of manipulations that are known to operate at an automatic, unconscious level. More precisely, by means of different priming procedures, it was tested whether unconscious activation of the concept of persistence could help to prevent loss of self-control. The central hypothesis in Chapter 3 was that activation of persistence induces automatic control behaviour and, consequently, does not require active involvement by the self. Since the strength model postulates that ego depletion emerges as a result of a conscious, active self-control (Muraven, Tice, \& Baumeister, 1998), it was expected that by unconsciously activating persistence, self-control performance will be enhanced and the occurrence of ego depletion may be circumvented.

Prior research has suggested that the direction in which people focus their attention while performing a task can have serious implications for task performance (see for instance Baumeister \& Showers, 1986; Heatherton, Polivy, Herman \& Baumeister, 1993; Jackson, Ashford, \& Norsworthy, 2006; Mercer, McNevin, \& Guadagnoli, 2004). Chapter 4 and chapter 5 present two studies that tested the role of attentional processes in active self-control operations. Chapter 4 examined the effect of focusing attention on the control demanding situation (internal focus) versus directing attention away from the self-control action (external focus). Indirect findings from past research imply that awareness of one's regulatory efforts is an important factor contributing to self-control failure (Karniol \& Miller, 1983; Yates \& Revelle, 1979). Consistent with this 
reasoning, we expected that self-control performance will suffer when attention is focused internally; i.e. on the control demanding action and the feelings that are present during this action. In contrast, we hypothesized that an external focus of attention will help to shift attention away from one's regulatory efforts, thereby increasing self-control performance. The effect of attentional processes on self-control performance was further elaborated in Chapter 5. Previous studies investigating the effects of self-focused attention on self-control performance have revealed a mixed pattern of findings; part of these studies highlight the self-control enhancing effect of self-focused attention (e.g., Macrae, Bodenhausen, \& Milne, 1998), while others illustrate the regulatory downside of self-directed attention (e.g., Gill \& Strom, 1985). The research presented in Chapter 4 was designed to provide more insight in these contradictory findings. It was reasoned that it is critical to differentiate between distinct types of self-focused attention. On the one hand, self-focused attention may involve the type of attention that was addressed in Chapter 4, namely attention directed towards the self-control action and internal sensations during this action. On the other hand, self-focus may also involve what is known under the term self-awareness. When self-focus concerns selfawareness, people become aware of the self's existence in the world and the salience of an internalized, social and/or situational standard is increased (Duval \& Wicklund, 1972; Carver, 2002). Chapter 5 attempted to establish a direct comparison between both types of self-focused attention. In order to isolate the effect of cognitive association, both types of self-focus were combined. More specifically, some participants were exposed to a manipulation that increased only self-awareness, whereas others received a manipulation that increased both self-awareness and cognitive association. We hypothesized that when self-focus involves self-awareness, self-regulatory performance will improve. Moreover, when self-focus involves both self-awareness as well as awareness of ones regulatory efforts, we expect it to result in worse performance compared to self-awareness alone. Importantly, Chapter 5 also examined the impact of both types of self-focus on the emergence of ego depletion.

In Chapter 6, the role of self-control and ego depletion is discussed in an applied context. More specifically, results presented in the present dissertation as well as other related findings are discussed in the light of criminality in order to provide more insight in the emergence of impulsive criminal behaviour. Moreover, based on this theoretical framework, practical guidelines for self-control improvement and prevention of impulsive crimes are offered.

Finally, Chapter 7 summarizes and concludes the content and results of this dis- 
CHAPTER 1 | General INTRODUCtION

sertation and provides directions for future research. Note that most of the chapters of the current dissertation were written independently from one another. As an inevitable consequence, the reader may sometimes experience overlap in terms of content. 


\section{CHAPTER 2}

Self-control related expectancies and self-control abilities

Published as: Alberts, H. J. E. M., Martijn, C., \& De Vries, N. K. (submitted). Self-control related expectancies and self-control abilities. 


\begin{abstract}
The research presented in the present chapter aimed to investigate the relationship between self-control related expectancies and self-control abilities. By means of three questionnaire studies it was investigated whether the extent to which people expect self-control to be dependent on limited energy relates to their self-reported self-control abilities. Results show that when people are more inclined to expect self-control to be dependent on limited energy, they report relatively worse self-control abilities.
\end{abstract}

\title{
Introduction
}

Many of us hold the idea that our energy is limited. When we come home after a demanding day at the office, a surprise visit of our mother in law may be more than we can take. In that case, lying on the couch seems the only attractive option. However, imagine the pleasant visit of a good friend after the same tiring day at the office. Suddenly, our tiredness has vanished. This example illustrates that mental exhaustion can be regarded as a flexible construct.

An empirical illustration of flexible limits comes from the field of biology. Ellen Langer describes the so-called "Coolidge effect" (1989, p. 30). When a male animal's sexual appetite is sated, and he has finished copulating, he needs a rest period. However, if a new female of the species is brought in, he will immediately resume mating (Dewsbury, 1981). This phenomenon clearly exemplifies the flexibility of exhaustion or, in a broader sense, the subjective nature of limits. According to Langer " ...fatigue and satiation do not necessarily occur at fixed points. To a large extent, mental and physical exhaustion may be determined by premature commitments; in other words, unquestioned expectations dictate when our energy will run out" (Langer, 1989, p. 135).

The idea that limits are flexible, contrasts with developments in the field of selfcontrol. Self-control entails the override or inhibition of competing urges, behaviours or desires (Barkley, 1997; Baumeister, Heatherton, \& Tice, 1994; Shallice \& Burgess, 1993). According to the strength model, the capacity to control ourselves is defined by our own restrictions (Muraven, Tice, \& Baumeister, 1998; Muraven \& Baumeister, 2000). These restrictions are a direct consequence of the idea that self-control draws on a fixed and limited resource, akin to strength or energy. After an act of self-control, the resource becomes temporarily depleted leaving the individual in a state of ego depletion. Therefore, performance on a second self-control act will be impaired 
because of lack of resources. Regarding the energy resource a number of assumptions are made. First, the resource is non-specific and used for a broad variety of self-control operations such as overriding or inhibiting impulses, regulating emotions, mood, thought or behaviour, etc. Second, the resource is limited; thus, the energy employed to inhibit the desire to eat a fattening snack is no longer available to suppress a negative emotion. For example, in one of their first studies on the ego depletion phenomenon, Baumeister, Muraven, Bratslavski and Tice demonstrated that food-deprived participants who were not allowed to eat desirable food, were less persistent on a subsequent, second self-control demanding task than food-deprived participants who were allowed to eat (1998; Exp. 1). Third, operating self-control is similar to operating a muscle. Just like a muscle, self-control becomes tired after use and needs rest to recover.

In the present article, we address the role of expectancies that people have about self-control. To continue the muscle-athlete analogy: does it matter whether the athlete thinks of his or her resources as limited or unlimited? In a study by Martijn, Tenbült, de Vries, Dreezens, and Merckelbach (2002), it was demonstrated that the notion of limited energy is relative and flexible, and that the perceived boundaries of one's self-controlling abilities are apt to change. In this experiment, participants watched a highly disgusting video excerpt and were asked to suppress their emotions. A control group watched the same video but received no suppression instructions. Immediately after, half of the participants who suppressed their emotions were informed that: "Often people think they have to rest after an effortful task. However, scientific investigations prove that this is not the case after emotional effort..." (Martijn et al., 2002; p. 446, Experiment 1). At a subsequent self-control demanding task, participants who received this information outperformed control participant and participants who received no such information. These results imply that people expect that exercising self-control leads to fatigue, and hence, to worse performance on a second self-control demanding task. If such expectancy is challenged, decline in performance is less selfevident and people may even achieve better.

The aforementioned findings imply that not the existence of a limited energy resource per se, but rather the belief or expectancy that self-control is dependent on limited energy contributes to self-control failure. Such expectancies may be called "mindsets", premature cognitive commitments, or an implicit or lay theory (Langer, 1989, Dweck, Chui, \& Hong, 1995; Furnham, 1989). The present studies were designed to gain more insight in the relation between self-control abilities and self-control related beliefs. Three questionnaire studies were conducted that aimed at testing whether 
there exists a relation between the extent to which people believe that self-control is dependent on limited energy and their self-reported self-control abilities. An individual who strongly believes that self-control is a matter of limited energy, is likely to be concerned with minimizing loss and the possible obstacles when exerting self-control. Such beliefs may cause one to quit self-control attempts sooner in order to minimize future failure. Holding the belief that self-control is dependent on limited energy may therefore have serious implications for self-control performance and long term goal achievement. Consistent with this reasoning, a recent series of studies of Mukhopadhyay and Johar (2005) showed that persons who think that self-control is an unlimited and malleable ability tend to set more goals than persons who believe their capacity is finite. Based on this line of thought, we expected that the more people are inclined to expect self-control to be dependent on (limited) energy, the less good self-control abilities they report.

\section{Study 1 \\ Method}

Participants. A total of 54 (19 men and 35 women) psychology students of the University of Maastricht participated in exchange for course credit. The age of participants ranged from 16 to 54 years $(M=22, S D=5.0)$.

\section{Measures}

Self-control Scale. To measure trait self-control, a Dutch translation of the Self-control Scale (Tangney, Baumeister, \& Boone, 2004) was administered to participants. The present study used the full version of this questionnaire, consisting of 36 items. Examples include "I do many things on the spur of the moment" and "Sometimes I can't stop myself from doing something, even if I know it is wrong". All questions were answered on a 5 point scale ranging from "not at all like me"(1) to "very much like me (5)". The Self-control Scale showed adequate reliability (Cronbach's $\alpha=.80$ ) in the present sample.

Energy Expectancy Scale. In order to measure the extent to which people consider self-control to be an energy consuming activity, a scale was developed that consisted of 10 propositions (see Table 1 ). These propositions were partly derived from a previous study by Martijn et al. (2002). Examples include "Controlling myself requires a lot of 
energy" and "A lack of energy causes me to become unable to resist temptations". The questionnaire was preceded by the following instruction: "The following statements concern self-control. Self-control means exerting control over the self. It entails suppression, change or continuation of acts, thoughts or emotions, even if this is in conflict with what you really want. An example of self-control is to continue studying, while you prefer to stop. Another example is saying "no" to a tempting piece of pie, because you are on a diet. Please indicate to what extent you agree with the statement below". The statement were rated on a 5-point scale ranging from "totally disagree"(1) to "totally agree"(5). Cronbach's $\alpha$ (.73) for the Energy Expectancy Scale (EES) demonstrated sufficient reliability for the current sample.

Table 1. Overview of Items of the Energy Expectancy Scale

\begin{tabular}{|ll|}
\hline 1. A lack of energy causes me to become unable to resist temptations \\
\hline 2. It costs me energy if I try not to think about something unpleasant \\
\hline 3. Often, the reason why I fail controlling my emotions is a lack of energy \\
\hline 4. It costs me a lot of energy when I am reading a boring textbook \\
\hline $\begin{array}{l}\text { 5. Often, after completing an unpleasant task, I am not able to complete another } \\
\text { unpleasant task }\end{array}$ \\
\hline 6. If I plan to do something that costs energy, I'll try to be well rested \\
\hline 7. If I am unable to control my temper, this is caused by a lack of energy \\
\hline 8. I find it hard to control myself when I am tired \\
\hline 9. Controlling myself requires a lot of energy \\
\hline $\begin{array}{l}\text { 10. Being able to successfully control ones emotions is rather determined by energy than by } \\
\text { motivation }\end{array}$
\end{tabular}

\section{Results Study 1}

Correlational analyses revealed a significant negative correlation between the scores on the ESS and the scores on the Self-control Scale, $r=-.31, p=.03$ (2-tailed), $M s=2.55$ vs. 3.29 , and $S D$ s $=.82$ and .42 , respectively. In accordance with our primary hypothesis, this finding implies that when people are more inclined to consider self-control as 
dependent on (limited) energy, they report relatively worse self-control abilities.

Factor analysis was conducted in order verify distinct theoretical dimensions underlying both scales. Principal component analysis with varimax rotation using a forced 2 factor model confirmed unique dimensionality of the scales by showing that the items of the Self-control Scale loaded the highest on Factor I, whereas the items of the ESS loaded highest and exclusively on Factor II. Analysis of variance showed $25.68 \%$ of the explained variance to be attributable to factor I and $10.0 \%$ to factor II.

\section{Study 2}

The findings of Study 1 suggest an association between people's beliefs about the operation of self-control and their self-control abilities. It was found that the less people are inclined to expect self-control to be dependent on (limited) energy, the better selfcontrol abilities they report. However, it is possible that these findings can be explained in terms of a sequence or order effect. Since the ESS was completed after the Selfcontrol Scale, it remains possible that participants who indicated that they possessed relatively low self-control abilities, tried to "make up" for this response by indicating that self-control is demanding and dependent on energy. According to this alternative explanation, higher scores on the ESS are not necessarily a reflection of true expectations, but rather an expression of justification for possessing relatively low self-control abilities. Study 2 aimed at investigating the impact of this possible sequence-effect by reversing the order of the two questionnaires. Thus, in Study 2, the Self-control Scale was administered after the ESS. In addition, since Study 1 concerned a relatively small sample size, Study 2 aimed to replicate the findings of Study 1 with a larger sample size. In Study 2, a total of 288 (78 men and 210 women) first-year psychology students of Maastricht University participated. The age of participants ranged from 18 to 27 year $(M=19, S D=2.9)$.

\section{Results Study 2}

Again, a significant negative correlation between scores on the ESS (Cronbach's $\alpha=$ .77 ) and the Self-control Scale (Cronbach's $\alpha=.83$ ) was found, $r=-.24, p<.001$ (2tailed), $M s=3.22$ vs. 2.39 , and $S D s=.49$ and .39 respectively. This finding is in line with findings of Study 1 and shows that stronger energy related beliefs of self-control are associated with decreased perceived (or reported) self-control abilities. 
In order to check for interrelated dimensions between the two scales, principal component analysis with varimax rotation using a forced 2 factor model was conducted. This analysis showed that the items of the Self-control Scale loaded the highest on Factor I, whereas the items of the ESS loaded highest and exclusively on Factor II. Factor I explained $24.81 \%$ of the variance, whereas $8.18 \%$ of the variance was explained by factor II.

\section{Study 3}

Study 2 replicated the findings of Study 1 by showing that higher scores on the EES were associated with lower self-reported self-control abilities. Study 3 was designed to test the robustness of this finding by selecting a different self-control measurement. Instead of measuring self-control abilities by means of the Self-control Scale, we used a questionnaire that assessed impulsivity. Impulsivity is a construct that is inherently related to self-control. Whereas self-control is the choice of a larger, more delayed outcome over a smaller-sooner outcome, impulsiveness is the opposite choice. Impulsivity has been defined as the counterpart of self-control (Ainslie, 1974; Green \& Myerson, 1993; Logue, 1988). In order to measure impulsivity, the Barratt Impulsiveness Scale-11 (BIS-11; Barratt, 1994) was used. This scale is a self-report measure of general impulsiveness and was chosen due to its widespread use and fine psychometric properties. The BIS-11 consists of 30 self-descriptive items that measure general impulsiveness using a 4-point scale ranging from "Rarely/Never" (1) to "Almost always/ Always" (4). Based on the findings of Study 1 and Study 2, we predicted that higher scores on the ESS would be positively associated with higher scores on the BIS-11. In Study 3, a total of 167 (42 men en 125 women) first-year psychology students of the Maastricht University participated. The age of participants ranged from 17 to 48 year $(M=20.5, S D=7.05)$.

\section{Results Study 3}

In accordance with our expectations, analysis revealed a significant positive correlation between the scores on the ESS (Cronbach's $\alpha=.74$ ) and the scores on the BIS-11 (Cronbach's $\alpha=.80$ ) $, r=.27, \mathrm{p}<.001$ (2-tailed), $M \mathrm{~s}=3.09$ vs. 2.14 , and $S D s=.57$ and .30 , respectively. This finding suggests that when people are more inclined to consider self-control as dependent on (limited) energy, they report higher 
levels of overall impulsiveness.

Principal component analysis with varimax rotation using a forced 2 factor model showed that the items of the BIS-11 loaded the highest on Factor I, whereas the items of the ESS loaded highest and exclusively on Factor II. This suggests that the two scales concern distinct theoretical dimensions. Factor I explained $13.98 \%$ of the variance, whereas $9.05 \%$ of the variance was attributable to factor II.

\section{General Discussion}

Indirect findings from previous studies on self-control and ego depletion suggest that expectancies about the operation of self-control may have an influence on self-control performance (Martijn et al., 2002; Mukhopadhyay \& Johar, 2005). More specifically, these findings suggest that self-control performance might be guided by the fact that people think that self-control depends on (limited) energy. The present research aimed to test this link more directly. A scale was developed to reflect participants' beliefs about the extent to which self-control is dependent on (limited) energy. In order to assess their self-control ability, participants completed the Self-control Scale (Tangney, Baumeister \& Boone, 2004) (Study 1 and Study 2) and the Barratt Impulsiveness Scale (Barratt, 1994) (Study 3). Correlational analysis of these list revealed that stronger energy related beliefs of self-control are associated with decreased self-reported selfcontrol abilities (Study 1 and Study 2) as well as higher levels of overall impulsiveness (Study 3). Whereas much of previous research on the link between beliefs and selfcontrol has examined beliefs about one's ability to exert self-control (see for instance Aspinwall \& Taylor, 1992; Brunstein \& Olbrich, 1985; Lent \& Hackett, 1987; Strecher, DeVillis, Becker, \& Rosenstock, 1986), these findings suggest that it may be worthwhile to consider people's beliefs about the operation of self-control as well. Future research may integrate the current findings with previous findings by investigating the relationship between beliefs about the operation of self-control and beliefs about self-control ability.

Although the present findings provide additional evidence for the existence of an association between energy related expectancies and self-control abilities, they do not provide information about the causality of this relation. Based on the findings from Martijn et al. (2002) and Mukhopadhyay and Johar (2005), it seems reasonable to assume that expectancies of self-control guide self-control performance. However, it also remains possible that expectancies are shaped by people's self-control abilities. 
Future research should therefore address the nature of causality and the possible interaction between energy related expectancies and self-control abilities. Moreover, it has to be acknowledged that the correlations that were found in the present studies were significant, but modest. The present findings may therefore best be considered as preliminary and may well function as a starting point for future research.

A limitation of the current research concerns the fact that self-control ability was measured by means of self-reports. Future research should investigate the impact of energy related expectancies in more depth by using one or more behavioural measures instead of relying solely on questionnaires. This is relevant from both a theoretical as well as practical point of view. After all, many health related interventions are aimed at improving behaviour that involves self-control, like for instance quitting smoking or condom use. According to the limited strength model, such interventions should be directed at increasing people's energy reservoir, by means of training (Muraven, Baumeister, \& Tice, 1999; Oaten \& Cheng, 2006). The present findings offer an alternative and suggest that it may be valuable to identify and systematically alter ineffective selfcontrol expectancies. 


\section{CHAPTER 3}

The role of automatic processes in overcoming ego depletion

Published as: Alberts, H. J. E. M., Martijn, C., Greb, J., Merckelbach, H. \& de Vries, N. K. (in press). Carrying on or giving in: The role of automatic processes in overcoming ego depletion. British Journal of Social Psychology. 


\begin{abstract}
Research has shown that repeated exercise of self-control leads to impaired performance on subsequent self-control tasks, a phenomenon labeled ego depletion. The current research investigated the influence of automatic processes on self-control performance. Study 1 showed that activation of persistence leads to stable self-control performance and may help to overcome effects of ego depletion. Initially depleted participants kept their physical self-control performances constant when primed with persistence. If such a prime was absent, self-control performance of depleted participants decreased indicating ego depletion. Using a different manipulation, these findings were replicated in Study 2.
\end{abstract}

\title{
Introduction
}

Self-control entails the override or inhibition of urges, behaviours or desires (Barkley, 1997; Baumeister, Heatherton, \& Tice, 1994; Shallice \& Burgess, 1993) and can be regarded as an essential ingredient for many successes and achievements in life. Recent developments in the field of self-control suggest that self-control is costly (Baumeister, Bratslavsky, Muraven, \& Tice, 1998). According to Muraven and colleagues, our capacity for self-control is limited (Muraven, Tice, \& Baumeister, 1998; Muraven \& Baumeister, 2000). These researchers state that self-control draws on a fixed and limited resource, akin to strength or energy. After an act of self-control, the resource becomes temporarily depleted causing a state of ego depletion. Therefore, performance on a subsequent self-control act will be impaired because of lack of resources. In the current paper, we focus on ways to overcome the effects of ego depletion and to improve selfcontrol performance.

The literature on ego depletion ascribes a number of features to the concept of energy resource. First, the resource is non-specific and used for a broad variety of selfcontrol operations such as overriding or inhibiting impulses, regulating emotions, making choices and so on. Second, the resource is limited, that is, the energy required to inhibit the desire to eat a fattening snack is no longer available to suppress a negative emotion. Third, the resource is similar to a muscle. Like a muscle, self-control becomes tired after use and needs rest to recover. Today, a wide range of studies have indeed shown that when people exert self-control, performance on a subsequent act of selfcontrol is likely to decrease (cf. Baumeister et al., 1998; Ciarocco, Sommer, \& Baumeister, 2001; Muraven et al., 1998). Together, the results of these studies illustrate 
that after repeated self-control, ego depletion seems to emerge automatically, causing almost inevitable failure of future self-control attempts.

Given the advantages of successful self-control, an important question is whether the negative effects of ego depletion can be prevented. To date, most studies on selfcontrol and ego depletion focussed on the conditions under which ego depletion occurs and only a few studies investigated if and how ego depletion may be prevented or counteracted. A first study by Muraven, Baumeister and Tice (1999) showed that self-control may be strengthened by a regime of training. Students were instructed to engage in self-control trainings (such as keeping food diaries or improving posture) for a 2-week period. After that period, students showed improved performance on a physical self-control measurement when compared to a control group. Martijn, Tenbült, De Vries, Dreezens and Merckelbach (2002) demonstrated another way to overcome the negative effects of ego depletion, namely by changing people's expectancies. In this study, participants watched a highly disgusting video fragment and were asked to suppress their emotions. A control group watched the same video, but received no suppression instructions. Immediately after, half of the participants who suppressed their emotions were informed that "often people think they have to rest after an effortful task. However, scientific investigations prove that this is not the case after emotional effort..." (Martijn et al., 2002; p. 446, Experiment 1). On a subsequent self-control demanding task, participants who received this information outperformed control participants and participants who received no such information. Ego depletion may also be compensated by increasing people's motivation to perform well on a subsequent self-control task. A recent series of experiments by Muraven and Slessarava (2003) demonstrated that depleted individuals persevered longer at a subsequent self-control task if they were led to believe that their persistence would be beneficial to others or to themselves. Depleted participants, who were not motivated, lowered their persistence and thus showed ego depletion.

To sum up, recent studies on overcoming ego depletion showed that self-control can be improved by various means such as training or explicitly increasing the benefits of enacting self-control. Although the above described studies use different manipulations to improve self-control, they share the way these manipulations are presented; all studies use explicit manipulations, requiring a certain amount of conscious attention and processing. An important question in the light of these findings is whether ego depletion can only be diminished or even eliminated by ways that depend on conscious attention or conscious processing. In other words, is it possible to circumvent the ne- 
cessity of conscious self-control?

In the present series of experiments we try to extent the current findings on selfcontrol and ego depletion by investigating whether the emergence of ego depletion can be reduced or even eliminated by means of manipulations that are known to operate at an automatic level and do not require conscious awareness to be effective. More precisely, we will investigate whether activation of the concept of persistence can help to prevent loss of self-control. As described by Baumeister et al. (2000) (see also Schmeichel et al., 2003, p. 87), ego depletion is not a sign of complete exhaustion, but a tendency to conserve a remaining amount of energy. However, Baumeister and colleagues do not mention whether strength conservation involves a conscious and deliberate decision or a more or less automatic process that does not necessarily require conscious awareness. In our opinion, the latter view is most likely to be true. In this view, the tendency to conserve resources can best be interpreted as a standard or default reaction after enactment of self-control. After an act of self-control people may automatically switch to a standby or "energy conservation" mode, that enables them to them to deal in the most careful way with the remaining amount of energy. If the reaction of depleted individuals to future self-control attempts is guided by this standby modus, then it seems worthwhile to know whether and how it is possible to switch to the "full operation" mode again. Previous research of Webb and Sheeran (2003) provided preliminary evidence suggesting that automatic processes can help to overcome the effects of ego depletion. The influence of automaticity was demonstrated by using implementation intentions (Gollwitzer, 1999). These are action plans and refer to specific goal behaviour as well as to the situational context in which the planned behaviour will be enacted (e.g., when I encounter situation x, I will initiate goal-directed behaviour y). Since behavioural control is overtaken by anticipated environmental cues, the process of control becomes automated. In two experiments it was consistently found that participants who formed implementation intentions that specified when and how to act during a depletion task, performed significantly better on a subsequent self-control task than depleted participants who did not form these implementation intentions. In other words, implementation intentions conserved self-regulatory resources.

In the present studies, we examined whether activation of concepts related to the maintenance of control behaviour, such as persistence, is also capable to offset the effects of ego depletion. Whereas previously discussed studies on self-regulation and automatic goal pursuit mainly addressed activation of self-regulatory goals (e.g., Bargh, Gollwitzer, Lee-Chai, Barndollar, \& Troetschel, 2001; Shah \& Kruglanski , 2002), the 
present studies focus on activation of self-regulatory behaviour. Instead of applying directly to one specific (self-regulatory) goal, persistence is clearly a behavioural construct that is (a-specifically) related to the maintenance of control behaviour in general. Activation of persistence may produce automatic control behaviour and, hence, circumvents the need for active and conscious self-regulation. Because of the absence of such need, this may be in particular useful for individuals who already suffer from resource depletion. In order to activate persistence, we used different priming procedures. In a series of experiments by Bargh, Chen, and Burrows (1996), it was already shown that when a trait construct or stereotype is activated by means of a priming procedure, behaviour changes accordingly, even in an unrelated context. That is, the thought of a specific behaviour was sufficient to increase the tendency to behave in that way. Likewise, in the present series of studies, we expect self-control related behaviour to be affected by activation of persistence. We expect that low depleted participants outperform high depleted participants. We also hypothesize that under condition of ego depletion, activation of persistence leads to relatively better self-control performance than no activation of persistence. In Study 1, low and high depleted participants were primed with persistence related concepts such as "perseverance" and "holding on" by means of a scrambled sentences procedure. Study 2 aimed to replicate these findings and activated persistence by means of a visual priming procedure.

\section{Study 1}

The aim of the first study was to investigate whether activation of "persistence" improves people's self-control performance. The first part of the study consisted of the manipulation of initial self-control induced by presenting participants either with 8 solvable, but extremely difficult labyrinths (high self-control) or 39 relatively easy labyrinths (low self-control). The labyrinths were selected on the basis of a pilot study. That study showed that difficult labyrinths were judged as significantly more difficult and almost impossible to solve as compared to easy labyrinths. Moreover, pilot participants expressed that they wished to quit solving difficult labyrinths after a few minutes and judged the task as exacting ${ }^{1}$. Although in a different form, a conceptually similar

${ }^{1}$ Labyrinths were pre-tested in an unrelated sample of 16 participants. Each participant received a 15-page booklet with a different labyrinth printed on each page: half of the booklets consisted of 15 extremely difficult labyrinths, the other half of 15 relatively easy labyrinths. Each participant was allowed to work exactly $10 \mathrm{~min}$ on the labyrinths and was then asked to evaluate the extent to which they judged the labyrinths as difficult and frustrating, and whether they had wanted to quit the task before the $10 \mathrm{~min}$ were over. Judgments were gathered on three 5 -point scales varying from $(1=$ not at all, $5=$ very much). In the difficult labyrinth condition, only one participants completed the first labyrinth, the other seven did not manage to solve one 
manipulation to induce ego depletion was used in a study by Baumeister, Bratslavsky, Muraven and Tice (1998) who exposed food-deprived participants to desirable but forbidden food for a fixed period of five minutes. Persisting with unattractive actions such as solving difficult labyrinths or refraining from tempting food, while one experiences the urge to quit, can be regarded as a task that requires self-control.

The second part of the study intended to increase the salience of self-control enhancing behaviour by means of priming. Half the participants were presented with a Scrambled Sentence Task (SST, Srull \& Wyer, 1979) including sentences related to persistence and perseverance, holding on, and so on, whereas the other half received a SST with neutral sentences (see also Araya, Akrami, Ekehammer, \& Hedlund, 2002). We chose for a SST because this is an implicit priming technique that reduces demand characteristics or other self-presentational concerns (Bargh \& Chartrand, 2000).

The third task of the study was a physical stamina task adapted from Muraven et al. (1998) and consisted of squeezing a handgrip. Although squeezing a handgrip seems primarily a matter of muscular strength, it has proven to be a successful measure of self-control in prior research (see for example Muraven, Tice, \& Baumeister, 1998, Study 1; Ciarocco, Sommer, \& Baumeister, 2001, Study 2). Because squeezing a handgrip becomes fatiguing after a short period of time, the person feels the urge to stop squeezing. Overcoming this fatigue and overriding the urge to quit, requires selfcontrol. We adopted the physical stamina measure as main dependent variable for two reasons. First, because most people think that a handgrip primarily depends on muscular strength, it is a relatively unobtrusive measure of self-control. Second, because squeezing a handgrip is quite distinct from the tasks we used in this experiment to induce depletion and to prime persistence, participants were less likely to suspect any connection between the tasks. In order to be able to control for individual differences in hand strength, handgrip performance was measured twice: before the labyrinths task and after the SST. Because of this baseline measurement, all participants started with a self-control task and thus exercised some self-control before starting with the actual self-control manipulation (easy vs. difficult labyrinths). Thus, strictly speaking the comparison was between groups of participants that differed in their level of depletion.

labyrinth within $10 \mathrm{~min}$. In the easy labyrinth condition, participants solved $13.2(S D=1.4)$ on average. This was reflected in the judgments of the difficulty level of the labyrinths ( $M$ difficult $=4.3, M$ easy $=1.5, t(14)=8,78, p<.01)$ and frustration $(M$ difficult $=3.1, M$ easy $=1.6, t(14)=3.14, p<.01)$. Moreover, participants who tried to solve difficult labyrinths indicated a stronger desire to quit the task earlier than allowed to ( $M$ difficult $=3.3, M$ easy $=1.9, t(14)=2.28, p<.05$ ). 
Our hypothesis was that participants who exercised initial self-control will perform better on a subsequent self-control task under conditions of high accessibility of persistence than under conditions of low accessibility of persistence.

\section{Method \\ Participants and procedure}

Eighty (72 women and 8 men) undergraduates of Maastricht University were randomly assigned to a 2 (self-control: easy vs. difficult labyrinths) x 2 (priming: neutral SST vs. persistence related SST) between-subjects design. Participants were individually tested in 30 min sessions and received a monetary reward for their participation. In order to minimize experimenter effects, the experimenter used a written protocol throughout the whole experimental procedure (all instructions to participants were equal). The experiment was approved by the standing ethical committee of our faculty.

After a short and neutral briefing on the study ("This study consists of a series of unrelated cognitive and physical tasks"), participants performed the first physical selfcontrol measurement that functioned as an individual baseline against which to compare the second physical self-control measure. The experimenter asked participants to place a handgrip in their dominant hand and inserted a coin between the two handles as the participant squeezed them together. Participants were instructed to squeeze the handgrip as long as possible. The moment the participant relaxed his or her grip, the coin fell out. The experimenter started a stopwatch at the moment he placed the coin between the handles and stopped timing when the coin fell out.

Following the first hand grip task, participants received a booklet with relatively easy labyrinths (low self-control) or extremely difficult labyrinths (high self-control). They were instructed to solve as many labyrinths as possible within $10 \mathrm{~min}$. After finishing the labyrinths, participants filled out the Brief Mood Inspection Scale (BMIS, Mayer \& Gaschke, 1988), containing 16 items and 6 items that were added to obtain additional data about the energy and tiredness component of participants' mood. In total, there were 8 ( 2 original BMIS and 6 extra) items pertaining to tiredness. Subsequently, we induced high accessibility of persistence by presenting a "verbal ability task". In fact, this was a scrambled sentence task (SST) that intended to prime participants with the concept of persistence. Participants unscrambled 25 scrambled sentences of which 15 were related to persistence (e.g., "Peter keeps going"); the other 10 were neutral and were not related to persistence or self-control (e.g., "Sam buys bread"). Persistence 
related and neutral sentences were presented in a random order that was the same for all participants in the persistence prime condition. Participants in the neutral priming condition received 25 neutral scrambled sentences. A pilot study revealed that the writing activity during the scrambled sentence task interfered with performance on the subsequent handgrip task. A majority of pilot participants indicated that their poor performance on the second handgrip task was due to their fast speed of writing during the SST. Therefore, we adapted the procedure and asked participants just to read the correct sentences aloud. After completing the SST, participants squeezed the handgrip for the second time, following the same procedure as for the first handgrip measurement. Next, participants once more completed the 8-item "energy and tiredness" mood scale. This scale consisted of two original BMIS adjectives related to energy and tiredness and the six additional items. Participants were finally asked to write down what they thought the experiment was about. The latter served to determine whether participants were aware of a possible influence of the SST. Participants were debriefed and thanked.

\section{Dependent measures}

The Dutch translation of the BMIS (Mayer \& Gaschke, 1988) was used to check for a possible difference in mood between participants in the easy and difficult labyrinth conditions (Cronbach's $\alpha=.85$ ). The BMIS is a selection of 16 adjectives tapping eight mood states: (a) happy (happy, lively), (b) loving (loving, caring), (c) calm (calm, content), (d) energetic (active, peppy), (e) fearful/anxious (jittery, nervous), (f) angry (grouchy, fed up), (g) tired (tired, drowsy), and (h) sad (gloomy, sad). The Dutch translation of the BMIS was scored on 16 5-point scales ranging from "definitely not feel" (1) to "definitely feel" (5). Six additional items were scored on the same 5-point scales in order to obtain additional information about the energy and tiredness component of participants' mood (Cronbach's $\alpha=.88$ ). These items were I feel fit/I feel that I can handle the world/ I feel lethargic/ I feel exhausted /I feel not like doing anything/ I feel dull. The total mood scale was completed directly after the first physical self-control measure. After the second physical self-control measure, a selection consisting of two original BMIS adjectives and the six additional items was administered, all pertaining to energy and tiredness (Cronbach's $\alpha=.90$ ).

In order to control for individual differences, physical performance was measured twice: at the beginning of the experiment and directly after the labyrinth task. Using a 
stopwatch, time (in msec) was measured that a participant squeezed the handles of the handgrip together.

\section{Results}

First, we checked whether any of the participants in the experimental SST condition were aware of the fact that the majority of these sentences were related to persistence. Not a single participant recognized the specific content of the sentences. Hence, none of out participants was aware of the main hypothesis of the experiment about the relation between activating persistence and reducing ego depletion.

Four participants had deviant scores on the first handgrip time $(z>3)$ and were recognized as outliers (outlier procedure cf. Ciarocco, Sommer, \& Baumeister, 2001). Therefore, further analyses described below included the data of the remaining 76 participants (easy labyrinths + neutral prime $N=19$, easy labyrinths + persistence prime $N=18$, difficult labyrinths + neutral prime $N=19$ and difficult labyrinths + persistence prime $N=20$ ).

In order to check the effectiveness of our self-control manipulation, we tested whether solving difficult labyrinths consumed more self-control than easy labyrinths. To test this, we compared within the neutral priming conditions the handgrip performance of participants who solved difficult labyrinths (high self-control) with the performance of participants who solved easy labyrinths (low self-control). As expected, an ANOVA with the mean scores of the two handgrip times as a repeated measurement factor, and initial self-control as between subject factor revealed a significant interaction effect of the two factors, $F(1,36)=7.37, p=.01, \eta^{2}=.17$. Under neutral priming conditions, participants who were exposed to the difficult labyrinths performed significantly worse than those who solved the relatively easy labyrinths ( $M$ s handgrip2 - handgrip $1=-13.54$ vs. 0.17 , and $S D$ s $=15.64$ vs. 15.51 ; resp.). Moreover, in order to investigate the alternative explanation that the above described differences in performance can be explained in terms of excessive performance on the first measurement or second measurement, we calculated correlations between the first and second measurement within the neutral priming condition for high and low initial self-control participants separately. Analysis ruled out this alternative explanation by revealing a comparable and significant positive correlation between the two measures in the high self-control condition, as well as in the low self-control condition $(r=.72, p<.01$ vs. $r=.71, p<.01$; resp.). 
One-way ANOVA with condition as independent variable and time on handgrip 1 as dependent variable checked for initial differences in handgrip strength and yielded a marginally significant effect, $F(3,75)=2.54, p=.06, \eta^{2}=.10$. Although only marginally significant, this effect might imply that differences in self-control performance at time 2 were the result of inter-group differences in the initial time measurement in stead of changes across groups. For this reason, in all further analyses of self-control performance, handgrip 1 was included as covariate in order to control for pre-existing personal differences in handgrip strength.

Multiple regression analyses were used to test the main hypothesis that under condition of ego depletion, activation of persistence leads to relatively better self-control performance than no activation of persistence. Multiple linear regression was utilized since it permits the specification of which comparisons to be made, without any loss or reduction of statistical power. In line with the two-way design of the study, both the factors initial self-control and priming were dummy-coded, comparing main effects of initial self-control and priming, plus their interaction.

Regression analyses revealed a main effect of initial self-control, $\beta=.20, t(75)=$ $2.36, p=.02$, indicating the standard ego depletion effect. That is, participants who solved difficult labyrinths (high initial self-control) performed worse at the second handgrip measure than participants who received easy labyrinths. A second main effect was found of type of priming, $\beta=-.23, t(75)=2.86, p<.01$. Participants who received a persistence prime performed relatively better at the second handgrip measurement than participants who received a neutral prime. The two-way interaction failed to reach significance, $\beta=-.05, t(75)=.60$. As can be seen in Table 1 , in which the handgrip performances per condition are summarized, the absence of the interaction is caused by the fact that both the difficult and easy labyrinth condition, profited from the persistence prime. 
Table 1. Self-control Performance in Study 1

\begin{tabular}{lccc} 
Condition & Time 1 & Time 2 & Change \\
\hline Difficult labyrinths + neutral prime & $53.13(21.30)$ & $39.59(20.37)$ & $-13.54(15.64)$ \\
Difficult labyrinths + persistence prime & $40.24(28.44)$ & $39.46(27.76)$ & $-0.78(19.17)$ \\
Easy Labyrinths + neutral prime & $31.28(21.69)$ & $31.46(18.40)$ & $0.17(15.51)$ \\
Easy Labyrinths + persistence prime & $38.60(27.00)$ & $50.32(29.33)$ & $11.72(23.33)$
\end{tabular}

Note. Values under Time 1 and Time 2 represent mean times (in sec) that participants squeezed the handgrip. Standard deviations are given in parentheses. Values under Change represent change in performance (in sec), from Time 1 to Time 2 .

One-way ANOVA revealed that solving easy versus difficult labyrinths resulted in a mood differences, $F(1,75)=9.45, p<.01, \eta^{2}=.11$. Participants who solved easy labyrinths reported a somewhat better mood than those who solved difficult labyrinths ( $M$ s $=3.68$ vs. 3.46 and $S D s=.30$ and .34 ; respectively). To investigate the possible influence of this mood effect on self-control performance, we included mood as a covariate in the previously described regression model.

Most important, the covariate mood was not found significant, $\beta=-.12, t(75)=$ $1.25, p=.22$. The remaining effects replicated our previous pattern; although the effect of initial self-control was non-significant, it directionally supported our previous pattern of results, $\beta=.12, t(75)=1.65, p=.11$. In addition, a main effect for type of priming was found significant again, $\beta=-.25, t(75)=3.10, p<.01$. No interaction effect was found, $\beta=-.029, t(75)=.35, p=.73$. These results suggest that differences in mood cannot fully account for the significant differences in self-control performance between conditions.

To investigate the possible influence of the priming procedure on participants' mood, we analysed the 8-item "energy and tiredness" mood scale, obtained directly after the second handgrip. This revealed that exposure to neutral or persistence primes did not lead to a significant difference in reported mood, $F(1,75)<1.0, \eta^{2}=.011(M s$ $=3.58$ vs. 3.49 , and $S D$ s $=.42$ vs. .45 ; respectively).

Furthermore, changes in mood were analyzed by computing the difference between the second "energy and tiredness" mood scale and the same 8 items of the first mood questionnaire. ANOVA did not result in any significant main or interaction effects (all $p$ 's $>$.05). The absence of changes in mood indicated that participants did 
not experience a change in mood during the course of the experiment.

In order to test the possible influence of mood changes on the performance on the physical self-control task, we included the difference between the second "energy and tiredness" mood scale and the same 8 items of the first mood questionnaire as a covariate in the regression analysis. Again, a significant main effect of initial self-control $\beta=.19, t(75)=2.29, p=.025$, as well as type of priming was found $\beta=-.23, t(75)$ $=2.85, p<.01$. In addition, no significant interaction effect was found, $\beta=.05, t(75)$ $=.60, p=.56$, whereas the covariate failed to reach significance $\beta=.01, t(75)=.09$, $p=.93$. These results indicate that the differences in physical self-control performance cannot be attributed to changes in mood.

\section{Discussion}

The data of Study 1 provide support for the hypothesis that increasing the accessibility of persistence may help to overcome effects of ego depletion.

Participants in the both the high and low self-control condition, kept their performance relatively stable as a result of priming. The findings indicate that initially depleted participants kept their physical self-control performances constant when primed with persistence. In the absence of such a prime, physical self-control performance of depleted participants went down, indicating the standard effect of ego depletion. Thus, although the interaction between $\mathrm{x}$ and $\mathrm{y}$ was non-significant, the pattern of means was in the predicted direction. In addition, the pattern of results of Study 1 suggests that the effect of ego depletion is not fixed or absolute, but nevertheless persistent. Low depleted participants who were exposed to a persistence prime outperformed high depleted participants who were exposed to the same priming manipulation. The finding that these groups differ can be held as an indication of fatigue or depletion. However, this effect of fatigue is not absolute. After all, high depleted participants who were primed with persistence performed better than high depleted participants who received a neutral prime.

Another important aspect with regard to the findings of Study 1 concerns the effect of priming of persistence on future self-control demands. Does the finding that no signs of ego depletion are present when people are depleted and exposed to the persistence prime indicate that ego depletion has been reduced or even eliminated, or does this finding implicate that a delay of ego depletion has occurred? According to the theoretical assumptions of the strength model, one may assume that depleted participants who 
were primed with persistence, should have used more of their (last) energy resources in order to be able to keep their self-control performance stable (cf. Muraven \& Slessarava, 2003). This explanation was exploratory examined in Study 2.

\section{Study 2}

In order to test the assumption that depleted participants who are primed with persistence use more of their (last) energy resources, the experimental procedure of Study 2 was extended with an additional task. When depleted, people display a shift towards preferring activities that require less effort (Holding, 1983). Moreover, as suggested by Muraven and Slessarava (2003), the decision to invest effort in a task strongly depends on a person's motivation for that task and his or her perception of the amount of energy left. Therefore, a task was selected that would be sensitive to such appraisals and participants were asked to indicate the preferred difficulty level for the last task. We reasoned that if priming of persistence causes people to invest more energy, this extra investment of energy should be reflected in a decreased willingness to invest in a third self-control task. As a consequence, people who were primed with persistence were expected to prefer a relatively lower difficulty of an additional task.

In summary, Study 2 was designed to provide converging evidence with the results of Study 1 and to exploratory investigate the effect of priming on future self-control demands. Although we relied on the same experimental procedure as in Study 1, some changes were made. Beside the addition of an extra task, we also used an alternative ego depletion manipulation and an alternative way to increase the accessibility of "persistence". In Study 2, ego depletion was induced by means of an attention control task and, instead of a verbal priming procedure, we used a visual priming procedure. In other respects, the experimental procedure of Study was similar to that in Study 1. As in Study 1, a depleting task was followed by a muscular endurance test. Performance on this physical self-control task represented the main dependent variable. We anticipated that depleted participants who were primed with "persistence" would perform relatively better on the second muscular endurance task than depleted participants who received no such prime. 


\section{Method \\ Participants and procedure}

Eighty undergraduates (60 women and 20 men) of Maastricht University were randomly assigned to one of four conditions. It consisted of a 2 (self-control: low vs. high) x 2 (type of prime: neutral prime vs. persistence prime) between-subjects design. All participants were individually tested in 40 min sessions. During the whole experimental procedure, the experimenter used a written protocol in order to minimize experimenter effects. The experiment was approved by the standing ethical committee of our faculty.

The experiment was presented as a series of unrelated tasks that tested participants' cognitive and physical abilities. After signing a consent form, all participants took part in the muscular endurance test to furnish a baseline against which to compare the second endurance task. Participants were instructed to hold a $1.5 \mathrm{~kg}$ weight as long as possible with their arm stretched in a $90^{\circ}$ angle with their torso while sitting on a chair in front of a computer screen. They were situated in such a way that their hand holding the weight was exactly $5 \mathrm{~cm}$ above a bell. When participants were not able to hold the weight in the earlier described position anymore, their arm dropped and consequently the weight hit the bell. The experimenter stopped timing when the bell rang.

Next, high self-control was induced through an attention control task. This 8 min task required participants to calculate and name the sum of digits presented on a computer screen. The difficulty of the calculations increased throughout the task. In the high attention-control conditions, these calculations involved various 2-digit numbers. Additionally, participants were auditively distracted while performing the task: by means of headphones, participants were exposed to a voice randomly naming one and two number digits and thereby interfering with their calculations. Thus, participants in the attention-control conditions had to focus their attention on the calculations on the screen and try to override their impulse to listen to the interfering noise. In the no attention-control condition, participants summed combinations of two 1-digit or 2-digit numbers without being exposed to auditory interference. Hence, participants could successfully perform the (relative easy) calculations without having to ignore the interfering stimuli. Similar manipulations of attention control have been successfully used to elicit depletion in previous studies of self-regulatory resources (Schmeichel et al., 2003; Faber \& Vohs, 2004). After finishing the calculations, participants filled out the first mood scale which included 16 items of the Brief Mood Inspection Scale (Mayer 
\& Gaschke, 1988) (Cronbach's $\alpha=.86$ ) and 6 additional items concerning the energy and tiredness component of participants' mood (Cronbach's $\alpha=.87$ ).

Subsequently, regulatory performance was measured again. Participants were seated in front of the computer screen and held the $1.5 \mathrm{~kg}$ weight with outstretched arm. At this point, the visual priming manipulation took place. Suddenly, while participants were holding the weight, the experimenter activated (by inconspicuously pressing a button) a screensaver which appeared on the computer screen. Participants were led to believe that this screensaver appeared by accident. In the persistence prime condition, the screensaver depicted a winning young man in a business suit and a logo saying: "www.you-can-do-it.com, wallpapers and screensavers". In the neutral prime condition, the screensaver showed a picture of a vase and a similar logo saying: "www. myscreensaver.com, wallpapers and screensavers". Next, participants completed an 8item "energy and tiredness" mood scale (see Study 1) (Cronbach's $\alpha=.84$ ).

Thereafter, participants were asked to perform the final task. The experimenter explained that the next task varied in level of difficulty and asked participants to choose a difficulty level ranging from very easy (1) to very hard (5). They were led to believe that the level they selected represented a corresponding task difficulty. In fact, all participants received the same task, irrespective of their choice of a particular difficulty level. This final task was presented in a booklet and consisted of two pairs of drawings that were almost the same, but with a certain number of differences. Participants' goal was to detect and report these differences.

Finally, a short questionnaire was used to determine whether participants recognized the true nature of the study. To assess the effectiveness of our depletion manipulation, participants were also asked to what extent they thought the calculation task was dull, easy, and required mental effort. After completing these final questionnaires, participants received a careful debriefing and they were probed for any questions or concerns.

\section{Dependent measures}

Most of the dependent measures were identical to Study 1. As in Study 1, performance on a physical task served as the main dependent variable. Beside the questionnaires concerning mood, we also included a manipulation check in the form of a short questionnaire about the depletion task.

To investigate whether prior exercise of self-control might cause people to prefer 
a task with lower difficulty, we registered the difficulty level participants chose. The selected level (1-5) on the last task represented the dependent measures on this task.

\section{Results}

No participants showed deviant scores on the first physical measurement $(z>3)$. Therefore, all 80 participants were included in the analyses.

As far as can be inferred from the description of the study given in their own words in the final questionnaire, none of the participants realized the true purpose of the experiment. In general, participants regarded "the link between difficult tasks and physical power" as the main purpose of the current experiment. On a 5-point scale, participants rated the distraction calculation task as relatively more exhausting than the no distraction calculation task $(M=2.9, S D=.70$ vs. $M=2.3, S D=.84$; resp. $) F(1$, $79)=13.12, p<.01, \eta^{2}=.14$. Meanwhile, participants liked the distraction $(M=3.3$, $S D=.98)$ and the no distraction calculation task $(M=3.6, S D=.63)$ equally well, $F(1,79)=2.5, p=.12, \eta^{2}=.03$. Likewise, the distraction calculation task was rated equally interesting $(M=3.3, S D=.84)$ as the no distraction calculation task $(M=3.4$, $S D=.86), F(1,79)<1.0, \eta^{2}=.004$.

In order to investigate whether the attention control task successfully induced ego depletion, we compared the physical performance of participants who solved difficult calculations with the performance of participants who solved easy calculations under neutral priming conditions. An ANOVA with the mean scores of the first and second physical measurement as a repeated measurement factor, and initial self-control as between subject factor revealed a significant interaction effect of these two factors, $F(1$, $38)=26.15, p<.01, \eta^{2}=.41$. This suggests that the attention control task successfully induced ego depletion (Ms physical measurement 2 - physical measurement $1=-5.07$ vs. -17.68 , and $S D s=6.11$ vs. 9.17 ; resp.). Additional analyses revealed a significant correlation between the two physical measures in the depletion condition, as well as in de no depletion condition ( $r=.97, \mathrm{p}<.01$ vs. $r=.98, p<.01$ respectively). This rules out the possibility that lowered performance on the second handgrip measurement should be attributed to relative extreme effort on the first measurement or vice versa. One-way ANOVA showed that the scores on the first physical task did not differ by condition, $F(1,79)<1.0, \eta^{2}=.002$.

Regression analyses were conducted in order to analyze the effects of initial selfcontrol (easy versus difficult calculations) and priming (persistence prime versus neu- 
tral prime) on self-control performance. As in Study 1, the main dependent variable, self-control performance, was measured by entering the second physical measurement as dependent variable, whereas the first measurement was included as covariate in the regression analyses. In order to compare main effects of initial self-control and priming and analyze their interaction, initial self-control and priming were dummy-coded.

Regression analyses revealed a main effect for initial self-control, $\beta=.11, t(79)=3.67$, $p<.01$, and a main effect for priming, $\beta=-.20, t(79)=6.66, p<.01$. More important, an interaction effect of physical self-control (holding the weight), initial self-control and priming was found, $\beta=.081, t(79)=2.7, p<.01$. Mean times on both physical self-control performances and their difference per condition are summarized in Table 2.

Table 2. Self-control Performance in Study 2

\begin{tabular}{lccc} 
Condition & Time 1 & Time 2 & Change \\
\hline Difficult calculations + neutral prime & $72.12(43.42)$ & $57.44(42.50)$ & $-17.68(9.17)$ \\
Difficult calculations + persistence prime & $77.04(37.42)$ & $77.35(28.41)$ & $.31(11.42)$ \\
Easy calculations + neutral prime & $74.90(31.54)$ & $69.83(28.61)$ & $-5.08(6.11)$ \\
Easy calculations + persistence prime & $72.66(34.53)$ & $75.51(29.92)$ & $2.85(12.94)$
\end{tabular}

Note. Values under Time 1 and Time 2 represent mean times (in sec) that participants squeezed the handgrip. Standard deviations are given in parentheses. Values under Change represent change in performance (in sec), from Time 1 to Time 2.

In order to further explore this interaction effect, dummy coding was used to compare self-control performance among the four groups. First, we compared the effect of priming within the two high initial self-control conditions. The two high initial self-control conditions were dummy coded as -1 and 1 , whereas the two low initial self-control conditions were both coded as 0 . Regression analysis revealed a significant effect, $\beta=-.20, t(79)=5.87, p<.01$. Depleted participants who were exposed to the persistence prime outperformed depleted participants who received a neutral prime. Next, the effect of priming for individuals who exerted low initial self-control was investigated by dummy coding the two low initial self-control conditions as - 1 and 1 and the two low initial self-control conditions as 0. Again, a significant effect was found, $\beta=-.12, t(79)=3.18, p<.01$, indicating that within the low initial self-control conditions, participants who were exposed to the persistence prime performed significantly 
better than those who received the neutral prime. Moreover, our main prediction was that under conditions of high self-control, handgrip performances will not suffer from ego depletion when offered a persistence related prime. Also, under conditions of low self-control, handgrip performances were also expected to remain stable. Thus, a decrease in handgrip times was only predicted for participants who exercised high initial self-control and who received a neutral prime. Therefore, regression analysis was performed in which the difference between the two handgrip performances of the high self-control, neutral prime participants (dummy coded as 3) was tested against the other three conditions (each condition dummy coded as -1). Results showed that decline in self-control performance of high self-control participants who were exposed to the neutral prime was significantly greater than the decline in performance of the other three conditions, $\beta=-.22, t(79)=7.19, p<.01$.

In contrast with Study 1 , we found that solving easy or difficult calculations did not result in a mood difference $\left[F(1,79)<1.0, \eta^{2}=.03\right]$. This makes it unlikely that differences in physical self-control measurements between the four conditions can be attributed to a mood effect.

A one-way ANOVA on the 8-item "energy and tiredness" mood scale questionnaire, taken directly after the second physical task, revealed that exposure to prompts did not lead to a difference in reported mood, $F(1,79)=1.3, p=.28, \eta^{2}=.05$.

Furthermore, changes in mood were analyzed by computing the difference between the second "energy and tiredness" mood scale and the same 8 items of the first mood questionnaire. ANOVA did not result in any significant main or interaction effects (all $p$ 's $>.05$ ). The absence of changes in mood indicated that participants did not experience a change in mood during the course of the experiment.

In addition, a regression analysis with the difference between the second "energy and tiredness" mood scale and the same 8 items of the first mood questionnaire as a covariate in the regression analysis revealed a significant main effect of initial selfcontrol $\beta=.11, t(79)=3.57, p<.01$, as well as type of priming $\beta=-.19, t(79)=6.41$, $p<.01$. In addition, a significant interaction effect was found, $\beta=.08, t(79)=2.65$, $p=.01$, whereas the covariate failed to reach significance $\beta=.01, t(75)=.09, p=.93$. Together, these findings indicate that differences in physical self-control performance are unlikely to be caused by changes in mood.

Finally, a similar ANOVA on preferred difficulty level yielded no significant main or interaction effect, $F(1,79)<1.0, \eta^{2}=.01$. In other words, participants did not differ in their preferred difficulty for the last task $(M=3.50, S D=.73)$. 


\section{Discussion}

In line with Study 1, results of Study 2 provided further evidence that activating "persistence" helps participants to overcome ego depletion. High depleted participants who were exposed to a "persistence" prime did not display the "standard" ego depletion pattern. That is, in these participants no decline in self-control performance was observed. In contrast, high depleted participants who received a neutral prime showed a significant drop in performance on the second self-control measurement.

Interestingly, whereas Study 1 revealed no significant interaction between priming and initial self-control, Study 2 did find an interaction effect. As discussed previously, the pattern of results of Study 1 implies that the ego depletion effect was not overruled completely. However, the interaction effect found in Study 2 suggests that ego depletion can be totally overridden by priming persistence. A possible explanation for these inconsistent findings may lie in the influence of the different priming manipulations. The fact that both high and low depleted participants profited from the persistence prime in Study 1, whereas only high depleted participants were influenced by the prime in Study 2, may imply a relatively stronger influence of the priming procedure used in Study 1.

Study 2 also investigated the effect of priming of persistence on future self-control demands. It was hypothesized that if exposure to a persistence prime causes people to invest more of their available energy, this should be reflected in a decreased willingness to invest in a subsequent self-control task. This was measured by asking participants to indicate the preferred difficulty level for an additional task. The results show that there are no significant differences among groups; all participants select about the same difficulty level. We recognize that this absence of differences between groups is a null-effect, which makes it difficult to draw definite conclusions. Yet, it does suggest that future self-control demands are unlikely to neither suffer nor benefit from exposure to the priming manipulation.

\section{General Discussion}

Successful self-control performance has repeatedly been shown to be dependent on previous acts of self-control. When people exert self-control, they generally perform worse on subsequent self-control tasks. According to the strength model, decreasing self-control performance is caused by a limited energy resource. Self-control consumes energy and leads to an automatic depletion of this limited resource, leaving less en- 
ergy available for further successful self-control. In the current paper, we questioned whether the automatic emergence of ego depletion can be reduced or even eliminated by means of manipulations that are known to operate at the same automatic level as the occurrence of ego depletion itself. Whereas previous studies have shown that selfcontrol can be improved by means of explicit manipulations that require conscious attention or processing, the current experiments investigated whether the same effects can be accomplished by ways that do not require conscious attention or awareness to be effective (Muraven \& Slessarava, 2003; Martijn et al., 2002). We investigated this by conducting two studies in which we activated the behavioural concept of persistence by means of different priming procedures. The results of both studies reveal that activation of the behavioural concept of persistence can have significant effects on self-control. Depleted participants who were primed with persistence performed significantly better on a subsequent self-control task than depleted participants who were exposed to a neutral prime (Study 1). These results were replicated in Study 2 by means of a different priming procedure. Priming of persistence caused initially depleted participants to overcome ego depletion: their performance on a subsequent self-control task remained equal. In contrast, depleted participants who were exposed to the neutral prime displayed the classic ego depletion pattern: their performance dropped significantly.

To summarize, in line with recent studies on ego depletion and self-control, we have demonstrated that enactment of self-control does not inevitably lead to poorer performance on subsequent tasks. Ego depletion can be overcome when a behavioural construct, that is essential to successful self-control, becomes activated. Moreover, the present studies show that no conscious awareness of this activation is required.

One important question concerns the underlying processes of the present findings. Although we have shown the influence of priming, little is known about the exact nature of these manipulations in relation to the self-control process. There are several possible explanations for the control enhancing effects we found. To begin with, activating persistence might somehow encourage people to use up their last energy resources. However, Study 2 showed that depleted individuals who were primed with persistence did not differ in their preference for difficulty on a third self-control task. In other words, even when priming causes people to invest more energy in a second control task, they are not more likely to choose a lower difficulty level on a third control task. This indicates that these people are not inclined to make things easier because of energy depletion or because of their need to save a last amount of energy. Moreover, 
one might argue that the effect of priming continues to work on the final self-control task. If this view is correct, differences should be observed because depleted people who benefit from the control enhancing manipulations should prefer a higher difficulty on a third control task than depleted people who are not primed. As the results of Study 2 made clear, this is not the case. Another possible reason for the absence of any differences between groups on final control tasks may be that priming does not fully deplete the energy resource and therefore does not lead to any significant difference in performance on or difficulty preference for a third task. However, the strength model is silent about the total amount of energy that people have. As a consequence, the possibility that a self-control task may not have depleted the energy system is difficult to test. Even when no differences in performance are found after exposure to a large number of depleting self-control tasks, one still might argue that total depletion is not reached. In other words, the strength model is not easy to falsify and questions arise when testability comes into play. For instance, it remains questionable whether a complete depletion of the energy resource is (a) ever the case and (b) can be manipulated within an experimental setting.

Another mechanism that may underlie our findings is that priming may restore the depleted self. In other words, activating persistence possibly replenishes the depleted self. In that case, self-control can best be regarded as an interactive process, in which the presence of a self-control enhancing state of mind functions as an important moderator. Clearly, this issue warrants further research.

Finally, the current findings may be explained in terms of implicit beliefs or expectancies. Heightened activity of the concept "persistence" might resemble guidance by positive self-control related beliefs. In other words, a person who is primed with persistence may behave in the same way as a person whose most dominant expectancies are characterized by motivation, self-efficacy and so on. In a prior study, we found some evidence that people distinguish between two classes of beliefs about exercising selfcontrol (Martijn et al., 2002, Experiment 2). The first class of beliefs characterizes selfcontrol as energy and comes close to the strength model of Baumeister and colleagues. Thus, people expect and believe that they have only a limited amount of energy available for their self-control operations and that they are likely to fail when demands are too high. Furthermore, laymen believe that for optimal performance one needs to be well-rested and free of other self-control demands. At the same time, albeit to a lesser extent, people adhere to the belief that self-control is primarily a matter of motivation: if you really want to, you can do it and if you really make an effort you can do much 
more than you think. Moreover, the distinction between these two classes of beliefs about self-control was recently corroborated by a comparable classification by Mukhopadhyay and Johar (2005) who showed that participants who think that self-control is an unlimited and malleable ability tend to set more goals. The issue is also reminiscent of the concept of self-efficacy (Bandura, 1986) in the sense that people who believe that self-control is a matter of motivation may be expected to experience more control in a self-control demanding situation than people who believe that their capacity to control themselves depends on their energy level. A similar impact of beliefs on selfcontrol was demonstrated in three different studies by Tice, Bratslavsky, and Baumeister (2001). It was shown that when participants believe their moods are susceptible to change, they respond to bad moods by increasing several impulsive behaviours such as eating, procrastination, and immediate gratification. However, when people are led to believe that their moods cannot be repaired by enacting impulsive behaviours, all these effects are eliminated. In sum, the above discussed studies raise the possibility that implicit theories or expectations underlie the current findings. However, future research should address this issue more directly by, for instance, considering the role and impact of interpersonal differences in self-control related beliefs and their impact on self-control.

\section{Limitations}

In present series of studies we used different supraliminal priming procedures in order to activate persistence related concepts. Although supraliminal priming procedures may reduce demand characteristic and other self-presentational concerns, they do not warrant complete elimination of these side-effects. A possible solution of this limitation may be offered by the implementation of subliminal priming procedures. With regard to future research, it seems worthwhile to consider whether the current findings can be replicated by the implementation of subliminal manipulations. The use of subliminal priming procedures may also provide a stronger test for the currently hypothesized influence of automaticity.

Another limitation of the present research concerns the way we tested the effect of priming on future self-control demands. In Study 2, this issue was addressed by asking participants to indicate the preferred difficulty level for an additional task. It was hypothesized that if exposure to a persistence prime causes people to invest more of their available energy, this should be reflected in a decreased willingness to invest 
in a subsequent self-control task. The results revealed that there were no significant differences among groups; all participants select about the same difficulty level. As discussed before, this suggests that the possible explanation that priming encourages people to use up their last energy resources seems unlikely. Although this finding is in line with previous research (Martijn et al., 2002), three critical points can be raised with regard to the finding of this null-effect. First, it is possible the sensitivity to detect interpersonal differences was not sufficient in current method of testing. Second, we used a method that was based on introspection. By asking people to select a difficulty level, people have to rely on their experienced depletion. However, their scores on the subjective mood scales show, that even when objective signs of depletion are present (e.g. worse performance on physical self-control tasks) people still report no significant differences on the fatigue related items. Therefore, it remains possible that the current results reflect the inability of participants to correctly report their mood or inner states of fatigue. Second, asking people to select a level may have caused them to adopt a middle course. In other words, the primary reason to select a particular level may be based less on their state of depletion and more on their need to conform to the standard norm. Future research should consider these limitations concerning the way we tested this hypothesis, by for instance measuring future self-control performance in stead of preference. Moreover, future research is likely to benefit from exploring alternative, more affirmative ways of testing whether participants expend their last energy resources after activation of persistence, for instance by implementing an additional exposure to persistence primes during the final task. 


\section{CHAPTER 4}

Attentional focus and self-control performance

Published as: Alberts, H. J. E. M., Martijn, C., Nievelstein, F., Jansen, A., \& de Vries, N. K. (submitted). Attentional focus and self-control performance. 


\begin{abstract}
In the present study, we addressed the influence of attentional control strategies on self-control performance. Our central hypothesis was that awareness of self-regulatory efforts negatively influences self-control performance. Indirect findings from past delay of gratification studies suggest that directing attention away from the control demanding action (external focus) improves self-control performance. The present research aimed to extend these findings by using an alternative, more active measure of self-control performance and by also testing the implications of increasing awareness of regulatory efforts. In order to increase awareness of regulatory efforts, we asked participants to pay attention to their arm and hand muscles that were involved while they were performing a physical self-control task (internal focus). It was found that participants who were distracted by a calculation task (external focus) while exerting physical self-control outperformed both participants who focused their attention on the physical self-control task (internal focus) as well as control participants. However, no significant difference in self-control performance was found between participants in the internal focus condition and control participants.
\end{abstract}

\title{
Introduction
}

Controlling oneself is often difficult: attempts to quit smoking are accompanied by withdrawal symptoms and intense craving, physical exercise is tiresome and may even hurt, and it can be immensely effortful to stay friendly to a blunt colleague or a difficult teenage daughter. What strategies may people employ to help them to control themselves successfully in order to reach their longer term goals?

In this paper, we concentrate on the type of attentional strategies that facilitate goal-directed behavior. More specifically, we address the role of attentional focus in self-control processes. In doing so, we differentiate between an internal and external focus of attention. Internal focus, also known under the term cognitive association (Goode \& Rot, 1993; Masters \& Ogles, 1998) can be described as directing attention to the self's (regulatory) efforts. For instance, a dieter who is constantly aware of the fact that he or she should not give in to the tempting food presented at a party, is focusing attention internally. The focus is on the feelings he or she experiences. This person pays attention to the relation between the self and the tempting stimulus and is aware that he or she is exerting self-control. In contrast, a dieter who neglects the presence of the tempting food at a party and seeks distraction by talking to other guests is focusing at- 
tention externally. External attentional focusing, also referred to as cognitive dissociation or distraction is defined as concentrating on aspects of the environment that are outside the self. In the current example, it involves attending to information unrelated to the self-control demanding stimulus or situation.

Previous research on attentional foci and effective self-control has mainly emphasized the benefits of external attentional focusing. For instance, in their delay of gratification paradigm, Mischel and Ebbesen (1970) posed children to the dilemma of choosing between an immediate, small treat or a delayed larger reward. An important observation in these studies was that some children were noticeably better able to delay gratification than others. It appeared that the success of these children was due to the usage of control strategies: they were able to shift their attention to other objects in the room, or dissociated themselves by focusing on the abstract properties of desired stimuli (thinking about sweets as little clouds, or imagining that they were just a picture and not real) (see also Rodrigues, Mischel \& Shoda, 1989). In a later study on delay of gratification by Mischel, Ebbesen and Zeiss (1972), the impact of attentional strategies was tested by externally supplying (instead of observing) the use of attentional strategies. These studies consistently showed that when children were given attentional strategies that helped to focus attention externally, they were able to wait significantly longer. In other words, by directing attention away from the control demanding action (external focus), children became less aware of their self-regulatory efforts, resulting in an improvement of self-control performance. With regard to internal focus of attention, this finding may also implicate that the awareness of ones self-regulatory efforts contributes to self-control failure. Past research findings indeed suggest that self-control performance is unlikely to benefit from constantly being confronted with ones self-control attempts. For instance, in a delay of gratification study by Yates and Revelle (1979), the immediate and delayed rewards were either exposed or concealed. The results showed that having rewards available for attention dramatically reduced children's ability to wait. Likewise, Karniol and Miller (1983) found that the more people think about the instant pleasure associated with immediate stimuli that trigger impulses to engage in a behavior, the harder it becomes to resist it.

In order to explain the processes that underlie the findings of the above described studies using the delay of gratification paradigm, Metcalfe and Mischel (1999) proposed a neural network model in which self-control occurs through an interaction between a hot, affective, "go" system and a cool, cognitive, "know" system. While the hot system develops early and is under stimulus control, the cool system develops later 
and is under self-control. According to the model, an external focus of attention can best be understood in terms of directing attention away from hot reward properties of a stimulus. Focusing attention externally leads to less input for the hot system, and thus to less influence of this system on behavior. The result is a facilitation of self-control. In contrast, focusing attention internally is expected to direct attention toward hot reward properties of a stimulus, thereby increasing input for the hot-system. As a consequence, the likelihood of a go reaction will be increased and self-control is more likely to fail (see also Strack \& Deutsch, 2004).

The purpose of the present study was to examine the influence of attentional control strategies on self-control performance. Our reasoning for the present study was based on indirect evidence from past research, namely that the awareness of one's regulatory efforts is an important factor contributing to self-control failure (Karniol \& Miller, 1983; Yates \& Revelle, 1979). In the current study, we aimed to test this rationale more directly. In doing so, we differentiated between an internal and external focus of attention. Past research findings using the delay of gratification paradigm have demonstrated that when children decrease awareness of their regulatory efforts by focusing attention away from the control demanding action (external focus), self-control performance improves. The present research aims to extend these findings. First, unlike past studies on attention focus and self-control in which participants were mainly children, the current experiment involved adult participants. Second, the delay of gratification paradigm entails a more passive form of self-control, namely waiting for a reward. The present study used an alternative, more active measure of self-control performance, namely physical endurance. Third, more important, we also considered the implications of increasing awareness of regulatory efforts (internal focus). The effect of an internal focus of attention on self-control performance has not yet -to our knowledge- been investigated directly and experimentally. Therefore, in order to gain more insight in the influence of attentional control strategies, the present study concerned a direct comparison of the effect of an external and an internal focus of attention on self-control performance. Based on previous observations (Mischel \& Ebbesen, 1970; Karniol \& Miller, 1983) as well as predictions made by the 2-system framework (Metcalfe \& Mischel, 1999) we anticipated that self-control performance is facilitated when attention is directed away (external focus) from the control demanding action. In addition, findings from previous research (Miller, 1983; Yates \& Revelle, 1979) provided indirect evidence that when attention is paid to the aspects related to the self-control action, a decline of self-control is more likely to occur. Therefore, we expected that par- 
ticipants whose attention is directed towards (internal focus) the control demanding action, will show poorer self-control performance compared to both participants who use external focus strategies as well as control participants.

As a measure of self-control we chose a physical exercise task, namely lifting a $1.5 \mathrm{~kg}$ weight as long as possible. Physical exercise tasks proved a successful measure of self-control in prior research (see for example Muraven, Tice, \& Baumeister, 1998, Study 1; Ciarocco, Sommer, \& Baumeister, 2001, Study 2; Martijn, Huijts, Alberts, Merckelbach \& De Vries, 2006). Because lifting a weight becomes fatiguing and even a little painful after a short period of time, the person feels the urge to stop lifting. Overcoming this fatigue and overriding the urge to quit, requires self-control. We adopted the physical stamina measure as main dependent variable because it is a relatively inconspicuous measure for self-control; most people think that a weightlifting primarily depends on muscular strength. Internal focus of attention was manipulated by instructing participants to pay close attention to physical sensation in their arm while lifting the weight. In this way, we ensured that during the self-control operation, attention was predominantly directed towards the self-control demanding action. Moreover, in order to increase participants' awareness of their self-regulatory efforts more directly, they were also presented with an anatomical drawing of an arm and were explained which specific muscles and tendons are typically involved when a person lifts a weight. An external focus was instigated by presenting participants with an intensive calculation task while they were lifting a weight. Since this calculation task was cognitively demanding, participants' attention was expected to be mainly directed to this task and to a far lesser extent to the control demanding action.

In order to control for interpersonal differences in physical strength, performance at the weightlifting task was measured twice; at the beginning and at the end of the experiment. Differences in performance at the first and second physical stamina measure formed the main dependent variable.

\section{Method}

\section{Design and Participants}

The design consisted of two experimental conditions in which type of focus (internal versus external) was varied and two comparison or base-line conditions. Self-control was measured by a physical endurance task, namely lifting a $1.5 \mathrm{~kg}$ weight and holding it as long as possible. In order to be able to furnish a baseline and to control for indi- 
vidual differences in physical strength, weightlifting was measured twice; at the beginning and at the end of the experiment. Eighty first year psychology students (60 men and 20 women, mean age $=20.5$ years, $S D=1.8$ ) of Maastricht University received course credit for their voluntary participation. Men and women were divided equally across conditions by means of a random procedure. The experiment was approved by the standing ethical committee of our faculty.

\section{Procedure}

Participants were tested individually in 20 min sessions. The experiment was presented as a study on mental and physical skills. The experimenter briefly explained the general procedure of the experiment after which participants signed a consent form. Next, participants were seated and asked to lift a $1.5 \mathrm{~kg}$ weight with their dominant arm. The experimenter started a chronometer at the moment the participant lifted the weight at a $90^{\circ}$ angle of the arm with the torso. The chronometer was stopped at the moment that a participant eased his or her arm and was not longer able to maintain the $90^{\circ}$ angle. After the first weight-lifting task, participant filled out a questionnaire that measured experienced fatigue and their mood. Next, type of focus was manipulated in the two experimental conditions. Participants in the internal focus condition were instructed to fully concentrate themselves on their arm and hand muscles that are involved when lifting a weight. To illustrate this, an anatomical illustration of an arm and hand was shown and the experimenter pointed at the muscles and tendons that are generally used to lift a weight. After this instruction, that lasted 3 min, participants lifted the weight again following the same procedure as in the first weight-lifting task.

Participants in the external focus condition received instructions about a cognitive calculation task that they were required to perform during the second weight-lifting task. This task was the Star Counting Task (SCT; De Jong \& Das-Smaal, 1990) that is designed to measure working memory capacity and requires the alternation of forward and backward counting. Each item in the Star Counting Test consists of a pattern of stars with plus signs, minus signs and empty spaces in between. The plus and minus signs specify the direction (adding or subtracting, respectively) in which the stars should be counted. Since the stars are presented in a table, empty spaces are added in order to prevent participants from counting multiple stars at once. Participants were instructed to start counting from a given number and sum the total number of stars. After completing each calculation, they had to indicate whether their answer matches 
or mismatches the number displayed at the top of each item. This number either represents the correct or an incorrect solution for the item concerned. Participants were asked to count aloud in order to make it possible for the experimenter to register the total number of stars counted. We used the SCT because its performance almost completely absorbs participants working memory and therefore leaves less capacity for other thoughts about the physical self-control. Thus, the crucial difference between the two experimental conditions was that participants in the internal focus condition were explicitly asked to concentrate themselves on their arm and hand muscles when lifting the weight whereas in the external condition such thoughts were made impossible.

The third condition was added to furnish a base-line against which weight-lifting performance in the two experimental conditions could be compared. Participants in the base-line weight-lifting condition received no specific focus instructions and simply lifted the weight again with a 3 min interval between the first and the second weightlifting task. The fourth condition served a base-line to compare the performance at the SCT. After the first weight-lifting task, participants in the base-line SCT condition were given the SCT during $3 \mathrm{~min}$ and the number of calculations was registered. After performing the SCT, they lifted the weight for the second time. In all four conditions, the time interval between the first and the second weight-lifting task was the same (3 min). Also, all participants used their same arm for both weight-lifting tasks.

After the second weight-lifting task, participants received two questionnaires to check their mood and their compliance to the instructions (manipulation check). Finally, participants were debriefed and thanked for their participation.

\section{Dependent measures}

All questionnaire items were answered on 4-point Likert scales ranging from "totally disagree"(1) to "totally agree"(4).

Mood. In order to check whether manipulations led to mood differences, participants completed the Brief Mood Introspection Scale (BMIS, Mayer \& Gaschke, 1988) directly after the second weight-lifting task (Cronbach's $\alpha=.90$ ). The BMIS consists of 16 adjectives, tapping eight mood states (happy, loving, calm, energetic, anxious, angry, tired and sad) with 2 adjectives each. Participants were instructed to rate "How well does each adjective or phrase describe your present mood". 
Subjective fatigue. In order to measure subjective fatigue, participants indicated their agreement on 8 statements (I feel tired / energetic / fit / drowsy / dull/ exhausted/ I don't feel like doing anything / I have the feeling I can handle the world). Reliability of this scale was sufficiently high (Cronbach's $\alpha=.80$ ).

Star counting task. On top of each item of the star counting task, a number was presented that either represented the correct or an incorrect solution for the item concerned. Participants were asked to indicate whether their answer matched or did not match this solution. However, since participants did not always complete their last item and often stopped halfway though, we reasoned that determining performance by the number of correctly indicated matches or mismatches implies omission of information. Therefore, performance was solely determined by the total number of stars participants were able to count during weightlifting time.

Manipulation check. Participants also completed one manipulation check question about what they were thinking about during the second physical measurement (During the physical task, I fully concentrated on my arm and hand).

Physical performance. Using a stopwatch, during the first (baseline) as well as second measurement time (in $\mathrm{msec}$ ) was measured that a participant lifted the weight.

\section{Results}

Self-control performance was represented by the difference in weight lifting time at the first and the second measurement. Five participants had deviant scores on the difference score of the two weight lifting tasks $(z>2)$ and were recognized as outliers. Hence, analyses described below included the data of the remaining 75 participants

Participants were asked to indicate what they were thinking of during the second physical measurement. Analysis revealed that the internal focus condition concentrated more on their hand and arm compared to the external focus condition, $t(37)=5.30$, $p<.001$ (2-tailed) ( $M$ internal focus $=3.25, S D=.85, M$ external focus $=1.89, S D=$ .74). A similar pattern of results was found for the internal focus condition versus the base-line weight-lifting condition, $t(36)=7.19, p<.01$ (2-tailed) ( $M$ base-line weightlifting condition $=2.5, S D=.79$ ) and for the base-line weight-lifting condition versus the external focus condition, $t(35)=5.84, p=.02$ (2-tailed). In sum, these findings implicate that our manipulations successfully directed participants' attention in the indicated direction.

An analysis of the fatigue and mood questionnaires revealed no significant differ- 
ences among the four conditions, $F(3,71)=.64$, ns and $F(3,71)=.84$, ns, respectively. This makes it unlikely that differences in physical self-control measurements between the four conditions can be attributed to a fatigue or mood effect.

One-way ANOVA showed that the scores on the first physical task did not differ by condition, $F(3,71)=.83, \eta^{2}=.034$. For the analyses of a possible difference in the handgrip time at the first and second measurement, we used an analysis of variance with condition as independent variable, handgrip time 2 as dependent variable and handgrip time 1 in the first questionnaire as covariate. Performance on the first handgrip was a significant predictor of performance on the second handgrip, $F(1,70)=121.52, p<.001, \eta^{2}=.64$. Also, we found a significant effect of condition, $F(3,70)=15.10, p<.001, \eta^{2}=.40$. Further inspection of the latter effect were established by means of planned contrasts. In order to compare the external focus condition with the other conditions, the external focus condition was coded as -3 , whereas the other three conditions were coded as 1 . In accordance with our expectations, participants in the external focus condition outperformed participants in the other three conditions, $t(71)=2.56, p<.05$ (2-tailed). Also, in order to compare the external and internal focus condition, the external focus condition was coded as -1 , the internal focus condition as 1 and both control conditions as 0 . It was found that participants the external focus condition performed significantly better than participants in the internal focus condition, $t(71)=2.01, p<.05$ (2-tailed). A comparison of the internal (coded -1) and base-line weight-lifting condition (coded 1 ) failed to reach significance, $t(71)=.51, p=.61$ (2-tailed). The means are summarized in Table 1 and show that participants in the external focus condition were able to keep their performance stable, while participants in the remaining conditions exhibited a decline in performance on the second physical measurement. 
Table 1. Self-control performance

\begin{tabular}{lccc} 
Condition & Time 1 & Time 2 & Change \\
\hline External Focus & $125(1.05)$ & $119(0.43)$ & $-6.0(0.33)$ \\
Internal Focus & $104(0.45)$ & $77(0.23)$ & $-27.0 *(0.34)$ \\
Control Physical measurement & $101(0.54)$ & $68(0.28)$ & $-32.0 *(0.35)$ \\
Control Star Count measurement & $106(0.35)$ & $80(0.24)$ & $-25.0 *(0.26)$
\end{tabular}

Note. Values under Time 1 and Time 2 represent mean times (in sec) that participants held the weight. Standard deviations are given in parentheses. Values under Change represent change in performance (in sec), from Time 1 to Time 2. Means with an asterisk differ significantly at $p<.05$.

In order to investigate whether stable self-control performance negatively interfered with performance on the calculation task, calculation task performance of participants who focused their attention externally was compared to performance of participants in the base-line SCT condition (control condition). Participants in the base-line SCT condition counted stars for a fixed period of time, namely three minutes. In the external focus condition, participants performed the task while lifting the weight. Since weightlifting time varied among participants, the time they spent on solving calculations varied as well. Therefore, for participants in the external focus condition, we calculated the total number of stars they were expected to count in 3 minutes. For instance, the total numbers of stars of a participant who lifted the weight for one minute and counted 30 stars during this period of time, was 90 (3 x 30). Subsequently, one-way ANOVA was conducted in order to compare the expected number of calculations of participants in the external focus condition with the observed number of calculations of participants in the base-line SCT condition. No significant difference was found, $F(1,35)=1.12$, $n s$. This finding suggests that relatively good self-control performance, as a result of an external focus strategy, did not negatively influence calculation task performance.

\section{Discussion}

This study tested whether self-control performance can be improved by employment of attentional control strategies. More specifically, it was hypothesized that self-control performance increases when one focuses attention externally, away from the control 
demanding action. In contrast, it was expected that this enhancement of self-control performance will not occur when focusing attention internally; on the control demanding action and the feelings that are experienced during a control attempt. In line with our expectations, it was found that participants who were distracted by a calculation task (external focus) while exerting physical self-control, performed significantly better than participants who focused their attention on the physical self-control task (internal focus). Interestingly, in contrast with our expectations, it was also found that performance of people who focused attention internally did not significantly differ from control participants' performance who did not receive any focus instruction. This absence of differences might imply that directing attention internally can be perceived as a default process or natural tendency that emerges when people exert self-control. Clearly, this issue warrants further research. Moreover, it was demonstrated that performing relatively well on a self-control task by using an external focus strategy, did not negatively influence performance on the distractive calculation task. Thus, focussing attention away from the control demanding action seems useful in improving selfcontrol performance, without suffering negative consequences for the task that one chooses to be distracted by.

The current findings are compatible with the hot-cool system theory (Metcalfe \& Mischel, 1999). When people adopt an internal focus of attention, they may become highly aware of their desire to quit performing the exerting self-control task. In this case, the positive aspects related to quitting (e.g., relaxation) are comparable with "hot" reward properties. Since increased salience of tempting aspects associated with giving up is expected to cause activation of the hot-system, self-control is more likely to fail. In contrast, by focusing attention externally, thoughts about the hot reward properties associated with quitting are less salient, implicating less activation of the hot-system. In line with the current findings, the expected result is relatively better self-control performance.

The present study illustrated that attention strategies can be considered as a useful way to improve self-control performance. However, at this point, it still unclear which psychological processes underlie the current findings. A possible explanation in terms of processes comes from the field of sport psychology. According to Wrisberg, Franks, Birdwell, and High (1988) "an internal focus of attention during exercise increases cognizance of the fatigue element of bodily sensation present during exercise". In the same way, an internal focus of attention during self-control performance may increase cognizance of the effort present during self-control attempts, whereas an external focus 
may reduce this cognizance. The awareness of one's efforts may initiate a chain of cognitive and evaluative processes that negatively contribute to self-control performance. In addition, the present results may also be explained in terms of regulatory strength. According to the strength model, controlling the self is psychologically demanding and results a state of so-called ego depletion (Baumeister, Bratslavsky, Muraven, \& Tice, 1998). When people control themselves, they use an amount of energy that is not available anymore for future self-control tasks, resulting in decreased performance on subsequent self-control attempts. In accordance with this model, we also found that people performed worse on the second physical self-control task when they were not focusing attention externally. The fact that participants who were distracted by the calculation task kept their self-control performance stable might imply that the usage of attention strategies can circumvent the effects of ego depletion. Focusing attention externally may circumvent the need for active and conscious self-control, thereby demanding less cognitive resources than focusing attention internally. Future research should address this issue more directly, providing more insight in the psychological processes that underlie self-control improvement by usage of attentional strategies. 


\section{CHAPTER 5}

Differential effects of self-focused attention on self-control performance

Published as: Alberts, H. J. E. M., Martiin, C., \& de Vries, N. K. (submitted). Differential effects of self-focused attention on self-control performance. 


\begin{abstract}
Previous studies on the influence of self-focused attention on self-regulation have revealed an inconsistent pattern of findings. Some studies suggest a control enhancing effect of self-focused attention; other studies rather suggest the opposite. The current investigation attempted to provide more insight in these divergent findings. A distinction was made between two types of self-focused attention, namely cognitive association and self-awareness. The present study used an ego depletion paradigm to test the hypothesis that the type of self-focus determines the effect of self-focus on regulatory performance. It was found that initially depleted participants who were exposed to a self-awareness manipulation kept their physical self-control performances constant. These participants outperformed both control participants as well as participants who were also exposed to the cognitive association manipulation. However, this control enhancing effect was absent when participants were highly self-aware but at the same time aware of internal states indicating self-regulatory efforts (cognitive association).
\end{abstract}

\title{
Introduction
}

Self-focused attention has repeatedly been shown to enhance self-regulatory behavior. For instance, in a study by Macrae, Bodenhausen, and Milne (1998) it was found that heightened self-focus enhanced spontaneous suppression of social stereotypes. Highly self-focused participants were better able to exert cognitive inhibition by producing less stereotypic descriptions compared to control participants (see also Carver \& Scheier, 1981, 1990; Monteith, 1993). Although these findings demonstrate a positive relation between self directed attention and performance, self-focused attention has also been found to impair (self-regulatory) performance (e.g., Gill \& Strom, 1985). For instance, in a recent study by Alberts, Martijn, Nievelstein, Jansen, and de Vries (2006) it was found that participants who focused attention on their inner sensations during a selfcontrol task, performed significantly worse on this task than participants who focused attention outward while performing the task.

In the present investigation, we address these seemingly contradictory findings. We propose that one way of explaining these divergent findings arises from the way the concept of self-focus is defined. Self-focused attention is often used as a synonym for objective self-awareness. Objective self-awareness is defined as becoming aware of the self's existence in the world and involves selective processing of information about the self (Duval \& Wicklund, 1972; Carver, 2002). Objective self-awareness has repeatedly 
demonstrated to enhance self-regulatory performance. The proposed mechanism that underlies this finding is that self-focused attention increases the salience of an internalized, social and/or situational standard. As a result, people are more inclined to adjust or regulate their behaviour in order to meet this standard. Imagine a dieter at a party. The presence of other guests at this party may increase the dieter's self-awareness. As the dieter is confronted by a waiter with a tempting tray of snacks, he or she may become very aware of the (internalized) standard that he or she should stick to the diet. Consequently, the self-aware dieter tries to meet the standard (not eating the food) by regulating his or her eating behaviour.

Past research on self-awareness has mainly focused on the differential effects of directing attention toward the self, with mirrors and cameras, and away from the self through various experimental distractions (see for instance Bailey, Leonard, Cranston, \& Taylor, 1983; Baldwin \& Holmes, 1987; Hutton \& Baumeister, 1992). The manipulations that have been used to induce self-focused attention cause people to become aware of themselves as they can become aware of any other person or object in the world. However, self-directed attention does not always involve the perspective taking that is induced by manipulations such as cameras, audience and mirrors. People may also direct attention inward, without becoming aware of the self as an object per se. Imagine the same dieter from the earlier mentioned example. Again, he or she is exposed to tempting food. While being confronted with this food, he or she may become aware of the desire to eat and the effort it takes to refuse the food while being exposed. In other words, instead of becoming aware of the self as an object, the person now becomes predominantly aware of internal states that indicate that one is exerting or has to exert self-control. The type of self-focused attention that is addressed here bears great similarity with a phenomenon that sport and exercise psychologist label cognitive association. Cognitive association is a type of self-focus in which attention is directed toward the sensations experienced during physical exertion (Goode \& Roth, 1993; Masters \& Ogles, 1998). It involves a narrowed type of attention in which one is predominantly aware of self-relevant internal sensation. Although cognitive association has been used as a synonym for self-focussed attention (see for instance Wrisberg, Franks, Birdwell, \& High, 1988), its negative effects on (self-regulatory) performance suggest that it differs from self-awareness.

Together, the previous findings suggest that self-focused attention is a complex and dynamic phenomenon, which does not always automatically cause improvement of regulatory performance. Although self-focused attention necessarily involves 
awareness of internal states, the nature and function of these internal states can differ. We argue that the stimulus by which attention is directed to the self, influences the nature and function of these internal states. In other words, stimuli that cause people to become objectively aware (e.g., mirrors and cameras), can influence internal states differently than stimuli that draw people's attention inward without making them objectively aware (e.g., presence of self-control demanding stimuli). With regard to the first example of the dieter, the presence of the guests can be regarded as a stimulus that primarily triggers objective self-awareness. Consequently, the dieter becomes aware of internal standards (refrain from eating) and is likely to notice any discrepancy between her present state and the salient standard (Duval \& Wicklund, 1972). The notion of this discrepancy implies awareness of an internal state, namely the current status (desire to eat) and the need to improve this state. In this case, awareness of the internal state(s) can be considered as a part of a regulatory progress that is affected by the salient standard. Here, internal state awareness serves as a monitor that keeps track of progress towards the intended goal (refrain from eating). This monitor function has also been referred to as a "test" phase (see TOTE model, Carver \& Scheier, 1981). In other words, the main function of the internal state is monitoring progress towards a goal or standard.

In the second example, the presence of the tempting food can be regarded as the stimulus that triggered awareness of internal states more directly (cognitive association), rather than awareness of the self as an object. In this case, the internal state (awareness of desire and effort to refuse) becomes the object of regulation. In other words, rather than being part of a regulatory cycle, now the internal state itself has to be regulated. The distinction between internal states as part of a regulatory cycle and internal states as object of self-regulation shows close resemblance to what is known as "state orientation" and "action orientation" (Kuhl, 1982; 1984). The theory of action control (Kuhl, 1982) proposes that in order to successfully perform intended behaviour, one needs to ensure that competing action tendencies are neglected. Two different states of the organism, namely state versus action orientation, are expected to determine the success of the process of action control. State orientation can be defined as a condition in which a person excessively focuses on his or her past, present or future state without attending to any action plan which may cause a change in the present situation. Action orientation occurs when an individual is attempting to implement an action or plan and focuses simultaneously on his or her present state, an intended future state, the discrepancy between these two and action alternatives which may change 
the current state into the intended future state. Now imagine that the dieter at the party is suddenly confronted by the waiter with a dish of snacks. The lovely smell of the snacks makes the dieter so aware of his or her effort to refuse the food (cognitive association) that it becomes almost impossible to resist the temptation. In this situation, the smell of the snacks can be regarded as a stimulus that triggers internal state awareness to the extent, that the regulatory enhancing effect of the standard is overruled by the concern to deal with the internal state. Whereas the internal state initially served as a part (test or monitor phase) of a goal oriented process (refrain from eating), the internal state (awareness of desire and effort to refuse) now attracts so much attention that regulation of this state becomes the primary concern. In other words, the initial action oriented process has become a more state oriented process. According to action control theory, the effectiveness of ones (regulatory) intentions decreases as a function of the proportion of processing capacity absorbed by state oriented processes.

The present study was designed as a direct test of the hypothesis that type of selffocus determines the effect of self-focus on regulatory performance. Where previous studies addressed the effect of either self-awareness or cognitive association on selfcontrol performance, the current study combined these two different types of self-focussed attention in order to establish a direct comparison between both types. In order to isolate the effect of cognitive association, some participants were exposed to a manipulation that increased self-awareness whereas others received a manipulation that increased both self-awareness and cognitive association. Based on previous findings, it was hypothesised that when a self-focus involves self-awareness, self-regulatory performance will improve. However, when a self-focus involves both self-awareness as well as awareness of ones regulatory efforts, we expect that this will result in worse performance compared to self-awareness alone.

In order to test these predictions, we used a so-called ego depletion paradigm. Self-control performance has been shown to decrease when people exert self-control repeatedly. The dominant explanation for this finding is that the exertion of self-control consumes some kind of energy, leaving the person in a state of ego depletion. Although a growing number of studies indeed prove impaired performance after selfcontrol, recent findings reveal that this decline in performance does not always occur. For instance, Muraven and Slessarava (2003) demonstrated that ego-depletion may be compensated by increasing people's motivation to perform well on a subsequent selfcontrol task. In addition, a study by Martijn, Tenbült, Merckelbach, Dreezens and De Vries (2002) showed that by changing depleted peoples' expectancies, their self-con- 
trol performance increased. Likewise, in the current experiment we expect that under conditions of depletion, participants who are highly objective self-aware outperform both participants who are aware of their regulatory efforts (cognitive association) as well as control participants.

The first part of the study aimed to manipulate the level of depletion. In order to induce a high level of depletion, participants were exposed to a self-control demanding task in the form of an attentional control task. Participants were asked to solve difficult calculations while they were auditively distracted during the task. Since participants had to pay attention to the calculations and needed to override their impulse to listen to the interfering noise, this task required self-control. A low level of depletion was induced by a version of the task in which relatively easy calculations were presented and no interfering stimuli were present. Since participants could perform the (relatively easy) calculations without having to control their attention by ignoring the interfering stimuli, this task was expected to require considerably less self-control. Similar manipulations of attention control have been used to elicit depletion in previous studies of self-regulatory resources (Schmeichel, Vohs, \& Baumeister, 2003; Faber \& Vohs, 2004; Alberts, Martijn, Greb, Merckelbach, \& de Vries, 2006). The second part of the study intended to manipulate type of self-focus. To do so, half of the participants were primed with sentences related to the self by means of a Scrambled Sentence Task (SST; Srull \& Wyer, 1979; see also Araya, Akrami, Ekehammer, \& Hedlund, 2002). In order to induce self-awareness, participants unscrambled 25 scrambled sentences that started with "I" (e.g., "I buy some bread"). Priming of self-related personal pronouns has been successfully used as a method to induce self-awareness in previous research (i.e., Brewer \& Gardner, 1996; Utz, 2004; Verplanken \& Holland, 2002). In order to induce cognitive association, a version of the task was presented in which 15 of these sentences were replaced with sentences that were specifically related to the self-control demanding action that was about to follow (e.g., "I feel my hand"; "I use my muscles"). The remaining half of the participants received 25 neutral scrambled sentences in which no self-relevant pro-verbs or self-control related words were present (e.g., "Henk buys some bread"). The only difference between the content of these sentences and the content of the self-awareness version of the task was the replacement of "I" with surnames. We choose for a SST because the nature of the task makes it suitable for subtle manipulation of the concept that is activated by the task (i.e., self-focus). In addition, SST is an implicit priming technique that reduces demand characteristics or other self-presentational concerns (Bargh \& Chartrand, 2000). 
The third task of the study was a physical stamina task adapted from Muraven et al. (1998) and consisted of squeezing a handgrip. Physical exercise tasks proved a successful measure of self-control in prior research (see for example Muraven, Tice, \& Baumeister, 1998, Study 1; Ciarocco, Sommer, \& Baumeister, 2001, Study 2). Because squeezing a handgrip becomes fatiguing and even a little painful after a short period of time, the person feels the urge to stop lifting. Overcoming this fatigue and overriding the urge to quit, requires self-control. We adopted the physical stamina measure as main dependent variable because it is a relatively inconspicuous measure for selfcontrol; most people think that sqeeuzing a handgrip primarily depends on muscular strength. In order to be able furnish a baseline and to control for individual differences in physical strength, handgrip performance was measured twice; at the beginning and at the end of the experiment. Differences in performance at the first and second physical stamina measure formed the main dependent variable.

\section{Method \\ Design and Participants}

The design consisted of two experimental and two control conditions. Whereas level of depletion was high in both experimental conditions, type of focus (cognitive association versus self-awareness) was varied. Two control conditions were added to the design in order isolate/compare the effect of the depletion and the focus manipulation. In the first control condition (high depletion - neutral prime), a high level of depletion was induced whereas a low level of depletion was induced in the second control condition (low depletion - neutral prime). In both control conditions, no focus of attention was induced. Eighty first year psychology students (60 men and 20 women, mean age $=23.1$ years, $S D=7.63$ ) of Maastricht University received course credit for their voluntary participation. The experiment was approved by the standing ethical committee of our faculty.

\section{Procedure}

The experiment was presented as a series of unrelated tasks that tested participants' cognitive and physical abilities. After signing a consent form, participants filled out the first mood scale, the Brief Mood Inspection Scale (BMIS; Mayer \& Gaschke, 1988). Next, all participants took part in the muscular endurance test to furnish a baseline 
against which to compare the second endurance task. The experimenter asked participants to place a handgrip in their dominant hand and inserted a coin between the two handles as the participant squeezed them together. Participants were instructed to squeeze the handgrip as long as possible. The moment the participant relaxed his or her grip, the coin fell out. The experimenter started a stopwatch at the moment he placed the coin between the handles and stopped timing when the coin fell out.

Next, high level of depletion was induced through an attention control task (Alberts et al., 2006). This 8 min task required participants to calculate and name the sum of digits presented on a computer screen. The difficulty of the calculations increased throughout the task. In the high attention-control conditions (high level of depletion), these calculations involved various 2-digit numbers. Additionally, participants were auditively distracted while performing the task: by means of headphones, participants were exposed to a voice randomly naming one and two number digits and thereby interfering with their calculations. Thus, participants in the attention-control conditions had to pay attention to the calculations on the screen and try to override their impulse to listen to the interfering noise. Whereas participants in the high depletion conditions performed the above described self-control demanding version of the task, participants in the low depletion condition were exposed to a less self-control demanding version of the task. This last mentioned group summed combinations of two 1-digit or 2-digit numbers without being exposed to auditory interference. After finishing the calculations, participants indicated their mood on a 5-point scale.

Subsequently, participants in the self-awareness condition were asked to complete a so-called "verbal ability task". In fact, this was a SST that intended to induce self-awareness. Participants unscrambled 25 scrambled sentences that started with "I". Participants in the cognitive association condition were also presented with this verbal ability task and asked to unscramble 25 scrambled sentences that started with "I". However, in order to narrow self-directed attention, 15 of these sentences were specifically related to the self-control demanding action (e.g., "I feel my hand"; "I use my muscles"). Participants in the two control conditions received 25 neutral scrambled sentences that did not contain any self-relevant words (e.g., "Henk buys some bread").

After completing the SST, participants squeezed the handgrip for the second time, following the same procedure as for the first handgrip measurement. After participants indicated their mood once more, they were finally asked to write down what they thought the experiment was about. The latter served to determine whether partici- 
pants were aware of a possible influence of the SST. Participants were debriefed and thanked.

\section{Dependent measures}

All questionnaire items were answered on 5-point Likert scales.

Mood. In order to test whether participants differed in their mood before they started with the experiment they completed the BMIS (Cronbach's $\alpha=.92$ ). The BMIS consists of 16 adjectives, tapping eight mood states (happy, loving, calm, energetic, anxious, angry, tired and sad) with 2 adjectives each. Participants were instructed to rate "How well each adjective or phrase describes your present mood". In addition, to check whether manipulations led to mood differences, participants were asked to rate their mood on a 5-point scale directly after the depletion manipulation and after the second handgrip performance.

Manipulation check. Participants were asked to indicate how boring, difficult and unpleasant they had experienced the calculation task.

Physical performance. Using a stopwatch, time (in msec) was measured that participants squeezed the handgrip.

\section{Results}

Self-control performance was represented by the difference in handgrip time at the first and the second measurement. Three participants had deviant scores on the difference score of the two weight lifting tasks $(z>2)$ and were recognized as outliers. Hence, analyses described below included the data of the remaining 77 participants.

As far as can be inferred from the description of the study given in their own words in the final questionnaire, none of the participants realized the true purpose of the experiment. In general, participants considered "the link between mood and physical power" as the main goal of the experiment. On a 5-point scale, the distraction calculation task was rated as relatively more difficult than the no distraction calculation task, $F(1,75)=30.99, p<.01, \eta^{2}=.29(M=3.23, S D=.93$ vs. $M=2.00, S D=.56$; resp.). Meanwhile, participants rated the distraction calculation task as more unpleasant than the no distraction calculation task, $F(1,75)=5.06, p=.03, \eta^{2}=.06(M=$ $2.93, S D=1.22$ vs. $M=2.25, S D=.97$; resp.). The distraction calculation task was rated equally boring $(M=2.81, S D=1.09)$ as the no distraction calculation task $(M=$ 


\section{6, $S D=1.05), F(1,75)=.47, \eta^{2}=.007$.}

In order to investigate whether the attention control task successfully induced ego depletion, we compared the physical performance of participants who solved difficult calculations with the performance of participants who solved easy calculations under neutral priming conditions (high depletion - neutral prime versus low depletion - neutral prime). An ANOVA with the mean scores of the first and second physical measurement as a repeated measurement factor, and level of depletion as between subject factor revealed a significant interaction effect of physical measurement and level of depletion, $F(1,37)=9.79, p<.01, \eta^{2}=.21$. This suggests that the attention control task successfully induced ego depletion. In other words, within the neutral priming conditions participants who were highly depleted (distraction) exhibited a more pronounced decrease in performance at the second physical measurement than participants who were less depleted (no distraction) (Ms handgrip time 2 - handgrip time 1 $=-9.66$ vs. -18.84 , and $S D s=21.54$ vs. 14.86; resp.). Additional analyses revealed a significant correlation between the two physical measures within the high depletion condition, as well as within the low depletion condition $(r=.73, p<.01$ vs. $r=.72$, $p<.01$ resp.). Hence, it seems unlikely that lowered performance on the second handgrip measurement should be attributed to relative extreme effort on the first measurement or vice versa.

Multiple regression analyses were used to test the main hypothesis that under condition of ego depletion, induction of self-awareness leads to relatively better selfcontrol performance than no focus or cognitive association. Multiple linear regression was utilized since it allows the specification of which comparisons to be made, without any loss or reduction of statistical power. The main dependent variable, self-control performance, was measured by entering the second physical measurement as dependent variable, whereas the first measurement was included as covariate in the regression analyses. In order to compare the effect of type of focus, the four conditions were dummy-coded.

Regression analysis was conducted with time on the second physical measurement as dependent variable, the four conditions as independent variable and time on the first physical measurement as covariate. Time on the first physical measurement was found as a significant predictor of time on the second measurement, $\beta=.77, t(76)=2.67$, $p<.01$. In order to further explore this effect, dummy coding was used to compare self-control performance among the four groups. First, we tested the effect of the selfawareness manipulation by comparing participants who were depleted and exposed 
to the self-awareness manipulation with depleted participants who were not exposed to any focus manipulation. The self-awareness condition and high depletion - neutral prime condition were dummy coded as -1 and 1 , whereas the other two conditions were both coded as 0 . Regression analysis confirmed our prediction and revealed that depleted participants who were exposed to the self-awareness manipulation performed significantly better than depleted participants who did not receive any focus manipulation, $\beta=.78, t(76)=59.11, p<.01$. Next, the effect of the cognitive association was investigated by dummy coding the cognitive association condition and high depletion - neutral prime condition 1 as respectively -1 and 1 , and the two remaining conditions as 0 . This time, no significant effect was found, $\beta=.75, t(76)=.56, p=.58$, indicating that when participants were exposed to the cognitive association manipulation, no control enhancing effects were observed. Finally, in order to compare the effect of selfawareness with the effect of cognitive association, both focus-conditions were coded as -1 and 1 , whereas the two control conditions were coded as 0 . Results showed that participants who were exposed to the self-awareness manipulation performed significantly better than those exposed to the cognitive association manipulation, $\beta=.79$, $t(76)=3.95, p<.01$. Mean times on both physical self-control performances and their difference per condition are summarized in Table 1.

Table 1. Self-control Performance

\begin{tabular}{lccc} 
Condition & Time 1 & Time 2 & Change \\
\hline Difficult calculations + cognitive association & $78.41(45.69)$ & $59.37(24.97)$ & $-19.04(29.09)$ \\
Difficult calculations + self-awareness & $72.20(42.36)$ & $80.30(41.41)$ & $8.97(18.74)$ \\
Difficult calculations + neutral prime & $61.55(29.82)$ & $51.89(27.35)$ & $-9.66(21.54)$ \\
Easy calculations + neutral prime & $76.98(20.31)$ & $85.77(19.95)$ & $8.79(14.86)$
\end{tabular}

Note. Values under Time 1 and Time 2 represent mean times (in sec) that participants squeezed the handgrip. Standard deviations are given in parentheses. Values under Change represent change in performance (in sec), from Time 1 to Time 2.

Analysis of the BMIS, taken at the beginning of the experiment, revealed that participants did not differ in their mood before they started with the experiment, $F(3,73)<1.0, \eta^{2}=.005$. Moreover, in order to investigate whether the depletion 
manipulation caused differences in mood, we analyzed scores on the second mood measurement under neutral priming conditions. It was found that solving easy or difficult calculations did not result in a mood difference, $F(1,38)<1.0, \eta^{2}=.005$. This makes it unlikely that differences in physical self-control measurements between the four conditions can be attributed to a mood effect. Likewise, scores on the last mood questionnaire, taken after the second physical measurement revealed no differences for participants who were exposed to the priming manipulation, $F(1,38)<1$, $\eta^{2}=$ .007. This indicates that the priming manipulation did not affect participants' mood. Furthermore, changes in mood were analyzed by comparing the difference second and the first mood measurement. ANOVA did not result in any significant main or interaction effects (all F's $<1$ ). This absence of changes indicates that participants did not experience a change in mood during the course of the experiment.

\section{Discussion}

Previous studies addressing the effects of self-focused attention on self-regulatory performance have revealed a non-consistent pattern of findings. Whereas some studies demonstrated a self-control enhancing effect of self-focused attention, other findings rather suggest the opposite to be true. The current investigation attempted to provide more insight in these divergent findings. It was argued that the type of self-focused attention plays a crucial role in explaining its effects on self-control performance. Selffocused attention may involve awareness of the self as an object (self-awareness) but may also involve a different process called cognitive association. The latter involves attention directed directly towards internal sensations and may lead to increased awareness of ones regulatory efforts, causing a negative effect on regulatory performance. It was hypothesized that the crucial difference between these forms of self-directed attention is the nature of the internal states to which attention is directed. When a stimulus induces awareness of the self as an object (self-awareness), appropriate standards have been found to become more salient and self-regulatory performance is enhanced (Duval \& Wicklund, 1972). The internal states that are present during self-awareness are expected to be related to the standard and function as a monitor: they are part of a regulatory process and keep track of progress toward the intended standard. In contrast, when people's attention is predominantly directed towards internal states instead of standards, these internal states may serve a different function: they become object of ones regulatory efforts. In order to test the hypothesized influence of internal 
states, an ego depletion paradigm was used in which one group of participants was exposed to a self-awareness manipulation whereas another group was given the same manipulation but was also made highly aware of their self-control related internal states (cognitive association). In line with previous findings, it was found that initially depleted participants who were exposed to a self-awareness manipulation kept their physical self-control performances constant. This group outperformed both control participants as well as participants who were also exposed to the cognitive association manipulation. This control enhancing effect was absent when participants were highly self-aware but at the same time aware of internal states indicating self-regulatory efforts (cognitive association). These findings show that self-focus does not always lead to increased self-control performance and suggest that the nature of internal states plays an important role in determining the effect of self-focused attention. When these internal states cause relatively greater stress on ones regulatory efforts, no control enhancing effect seems to emerge.

The current findings and theoretical assumptions may also provide some new insights in the ego depletion phenomenon. The fact that performance of depleted participants did not significantly differ from depleted participants who were also exposed to the cognitive association manipulation is in line with previous findings (Alberts et al., 2006) and implies that depletion may cause people to involve in cognitive association. By linking the current findings to the theory of action control (Kuhl, 1982), we already suggested that cognitive association may reflect state orientation. Depleted participants as well as participants who become very aware of their regulatory efforts may focus excessively on the present state, namely exerting self-control. Or, in terms of action control theory, ego-depleted people may become more state oriented than action oriented. According to action control theory, an important reason why state orientation often inhibits performance is because the excessive focus on the present withholds people from making action plans. If true, this implies that by making action plans, selfcontrol performance should improve and the effects of depletion should be less evident. Indeed, research by Webb and Sheeran (2003) found that by forming implementation intentions (action plans that detail when, where, and how to proceed toward a goal), it is possible to enhance self-regulatory performance and overcome the effects of ego depletion (see also Oyserman, Terry \& Bybee, 2002). In addition, a recent study by Hofmann, Rauch and Gawronski (2006) addressed the impact of automatic attitudes, dietary restraints standards and ego-depletion on eating behaviour. They found that the behavioural impact of personal standards was reduced under conditions of ego 
depletion. More specifically, when self-control resources were high, candy consumption was mainly predicted by participants' dietary restraint standards. However, when self-regulation resources were low, rather than dietary restraint standards, candy consumption was strongly predicted by participants' attitudes towards candies. From this perspective, the current finding that self-awareness helped to overcome the effects of ego-depletion may implicate that self-awareness helps people to become more action oriented by making present goals or standards more salient. These goals or standards then function as an intended future state, causing the individual to regulate the present state into the desired future state.

We have mainly argued that the nature and function of internal states play a key role in explaining the effects of self-focused attention on self-regulation. However, it must be acknowledged that the present study offered no direct measure of the supposed difference in internal states. For instance, if internal states of self-aware individuals indeed serve as a monitor of a goal oriented process (action orientation), than a difference in availability of certain goal-related concepts should be observed compared to individuals who are more involved in cognitive association (state orientation). Clearly, future research should benefit from implementing ways to measure these proposed differences. In addition, the present investigation revealed that under conditions of cognitive association, no self-regulatory enhancing effects were observed. However, internal states that become salient during cognitive association mainly concern a specific type of self-relevant information, namely ones regulatory efforts. In order to gain a better understanding of the relationship between self-focused attention and internal states, future research should address the impact and possible interplay of internal states with a different nature as well. 


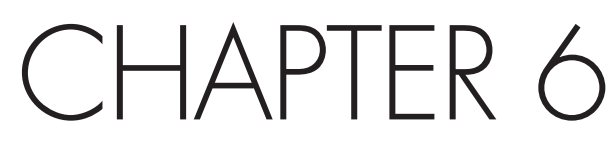

Understanding impulsive crime: The role of state self-control

Published as: Alberts, H. J. E. M, Panahi, R. M. S., \& Martijn, C. (2006). Understanding impulsive crime: The role of state self-control. Verlag für Polizeiwissenschaft, 3, 30-43. 


\begin{abstract}
The aim of the present chapter is to provide more insight in the psychological processes underlying impulsive criminal behaviour. More specifically, an overview of psychological findings and theories is presented in order to gain more knowledge about why and under which conditions impulsive crimes are likely to occur. Instead of focusing on stable, personality related factors, the current article mainly addresses a state oriented approach, by identifying factors that influence the momentary occurrence of impulsive (criminal) acting. Self-control is introduced as a central psychological construct. Research findings and theories addressing momentary self-control failure are linked to the emergence of (impulsive) criminal behaviour. In addition, guidelines for preventing failure of impulse control and improvement of self-control performance are provided.
\end{abstract}

\title{
Introduction
}

Criminality is a widespread and serious problem that confronts our society. The negative consequences of criminality are not restricted to the victim(s) involved, but also affect society as a whole, for instance by reducing feelings of safety and trust. A common way to prevent and fight against the emergence of criminality is by focusing on external control, ranging from gun laws to prison terms. In some cases, especially cases involving organized crime or planned criminal activity (referred to as intentional crime; Stark, 1989), the deterring effect of external control attempts, like imprisonment, may actively prevent people from committing crimes. From a psychological perspective, there is also an alternative way to prevent and fight against the emergence of criminality, namely by gaining more insight in the causes of and processes underlying criminal behaviour. In this case, the focus is more on internal control.

Many social problems and discontents, such as assault, sexual abuse and robbery, can be summarized under the term impulsive crimes (Stark, 1989). In contrast to intentional crimes, these crimes are characterized by a tendency to give in to violent or other disastrous impulses. When committing an impulsive crime, people lose control and behaviour is guided by their dominant impulses. Since impulsive behaviour is based on sudden desires and impulses rather than arising from careful and deliberate thought (Baumeister, Heatherton \& Tice, 1994), the consequences of the act often become apparent after the crime has been committed. For instance, most people will agree that impulsively attacking someone in response to a verbal insult is certainly not 
worth going to prison. However, the consideration of this long-term context will often not occur until the violent act has been committed (see also Berkowitz, 1978). Clearly, in these cases, the introduction of severe punishments may not always be the most optimal way to prevent occurrence of criminal acts. In stead, we suggest that attempts to prevent and counteract the occurrence of impulsive crimes, are likely to benefit from knowledge about the causes and processes concerning the deviant behaviour. In line with this, Stark (1989) suggested that in contrast to intentional crimes, which have rates well-predicted by societal variables such as population turnover rates and proportion of churchgoers, impulsive crimes are better explained by psychological variables. Moreover, taking these psychological variables into account by identifying why some people posses a higher propensity to engage in impulsive crimes and when (under which conditions) impulsive crimes are likely to be committed, not only leads to a better understanding of criminal behaviour, it also helps to formulate concrete prevention guidelines.

\section{Self-control as an essential capacity}

The commitment of an impulsive crime reflects the inability to control oneself successfully. In psychological terms, impulsive crimes are considered as manifestations of selfcontrol failure. Self-control can be defined as the override or inhibition of urges, behaviours or desires (Baumeister, Heatherton, \& Tice, 1994; Shallice \& Burgess, 1993). For instance, a person who is seriously offended is likely to experience anger and may even feel the urge to aggressively attack the offender. In order to prevent him or herself from harming the other, the offended person must exert self-control; he or she needs to control destructive emotions and the urge to physically express aggression.

Another important aspect of self-control concerns its relationship with goals. Selfcontrol is needed in order to pursue goals successfully. Goals can be conflicting; short term, proximate goals may negatively interfere with the accomplishment of long term goals. In order to accomplish the (often more rewarding) deferred long term goal, people have to overcome their impulse to enjoy the proximate goals. The eventual rewards that accompany long term goals may be far greater than the immediate reward of a proximate goal, but the immediate reward is often more tempting simply because it is immediate. For instance, after release from prison, ex-detainees may decide to start up an honest life and keep to the straight and narrow. While they search for jobs, they soon notice that their criminal record reduces their chance to find a job. As a re- 
sult, their financial situation becomes perceptibly deteriorating. In the past, financial problems would prompt them to gain money by committing a theft; their intention to keep to the straight and narrow, however, may prompt them to override this response and keep searching for potential jobs. In other words, in order to successfully choose for the long term goal (keeping to the straight and narrow), one needs to control the urge or desire to fullfil the short term, proximate goal (immediately gaining money by committing a theft). Clearly, in the long run, pursuing the long term goal is far more beneficial than giving in to the temptation of the short term goal. The benefits of long term goal pursuit in the context of crime is well reflected by the fact that most financial benefits gained by crimes are relatively small and short lasting (Gotfredson \& Hirschi, 1990; Katz, 1988). Obviously, however, people often fail to successfully pursue their long term goal and succumb to the attraction of short term satisfaction.

Although self-control is often used as a synonym of self-regulation, we will use it here in a more narrow sense. Self-regulation refers to a broad, regulatory cycle of comparing the self against a relevant standard, operating on the self to reduce a possible discrepancy, testing again and continuing the process until a test reveals that the standard has been reached, whereupon the person exits in the circle (test, operate, test, exit (TOTE); cf. Carver \& Scheier, 1981). According to Baumeister and Exline (2000), self-control refers to the "operate" phase, in which the self alters itself in order to move closer to the desired standard. This active and conscious process aims to reduce the risk that any thought, feeling or behaviour that might be incompatible with the current intention could take over. Depending on the situation, self-control can be defined in terms of activation or inhibition of the self. Continuing to perform an unpleasant task illustrates a form of self-control which requires activation of the self. On the other hand, resistance to temptation during recovery from an addiction clearly involves inhibition of the self.

Self-control can be conceptualized as a relatively stable personality trait and as a behaviour that varies in different situations. Trait self-control refers to an individual's general self-control capabilities. Trait self-control develops during lifetime and concerns a relatively stable personality characteristic. State self-control, on the other hand, applies to a specific situational context. State self-control can be defined as a temporary state that varies with intensity. Trait self-control is associated with state self-control since state-self-control can be regarded as an observable manifestation of trait self-control. 


\section{The role of trait self-control: A general theory of crime}

Today, a large amount of studies have mainly focused on why some people posses a higher propensity to engage in (impulsive) crime. Many of these studies are guided by the assumptions made by the influential "General Theory of Crime" (Gottfredson \& Hirschi, 1990). According to the General Theory of Crime, the main reason why some people posses a higher propensity to engage in criminal or analogous behaviour (e.g. smoking, gambling, excessive drinking) is because of interpersonal differences in self-control abilities. The theory mainly focuses on trait self-control and proposes that individuals can be arrayed on a continuum of self-control; those who possess low self-control abilities are expected to have a higher likelihood of criminal activity than those with strong self-control abilities. Low self-control increases the chance that an individual will be unable to resist the easy, immediate gratification that crime and analogous behaviour offers in daily life. According to Gottfredson and Hirschi, people who lack self-control abilities are characterized as "impulsive, insensitive, physical (as opposed to mental), risk taking, short-sighted and non-verbal". Self-control is considered as an essential construct, however, the theory also states that low self-control only results in criminal behaviour when the opportunity to engage in the behaviour is present. Although not without criticism and controversy, a relatively large number of studies have indeed found support for this core assumption of the theory and showed that poor self-control (and opportunity) related to various sorts of criminal and analogous acts, across different countries (Arneklev, Grasmick, Tittle, \& Bursik, 1993; Cochran, Wood, \& Sellers, 1998; Forde \& Kennedy, 1997; Gibbs \& Geiver, 1995; Grasmick, Tittle, Bursik, \& Arneklev, 1993; Higgins \& Makin, 2004; Junger, West, \& Timman, 2001; Longshore \& Turner, 1998; Nagin \& Paternoster, 1993; Piquero \& Tibbetts, 1996; Polakowski, 1994; Sellers, 1999; Vazsonyi, Pickering, Junger, \& Hessing, 2001; Wood, Pfefferbaum, \& Arneklev, 1993).

Since conduct problems first emerge early in life, the General Theory of Crime states that interpersonal differences self-control are established in early childhood socialization. Gottfredson and Hirschi (1990) emphasize the importance of family and the family environment. They argue that the family plays a vital role in ensuring the child adheres to social norms and non-deviant values. The most effective way to socialize a child correctly is to monitor, recognize, and punish the child's deviant behaviour. Children whose parents supervise and punish their misconduct are likely to develop the self-control needed. These children will not only be better able to resist the easy gratification offered by misbehaviour, they will also develop the will to succeed in so- 
cial institutions (e.g. school and job market). Several studies have indeed found some support for these predictions related to socialization (see Cochran et al., 1998; Gibbs, Giever, \& Martin, 1998; Hay, 2001; Polakowski, 1994).

\section{The role of state self-control: investigating momentary failure}

The fact that the General Theory of Crime has occupied an essential presence in criminology today as well as the large number of studies that support the assumptions of the theory confirm the importance of self-control in criminal behaviour. Moreover, when explaining interpersonal differences in criminal behaviour, these studies illustrate the value of taking interpersonal differences in trait self-control into account. However, limitations become apparent when investigating the conditions under which self-control failure or impulsive criminal acts are likely to occur. Trait oriented approaches of self-control, like the General Theory of Crime, are not capable of specifying when breakdown of self-control is more likely nor do they explain why a specific situation may lead to urge driven behaviour. In order to gain more insight in the processes and causes that underlie momentary failure of self-control, a state oriented self-control approach is needed. This approach will be the main focus of the current paper. More specifically, recent (social) psychological research findings and theories addressing momentary self-control failure will be linked with the emergence of (impulsive) criminal behaviour. In addition, based on recent findings, guidelines for preventing failure of impulse control and improvement of self-control performance will be provided.

\section{Self-control failure and ego depletion}

In many cases, failure of self-control can be considered relatively harmless. For instance, a dieter who gives in to his urge and fails to refuse a fattening snack is unlikely to cause any societal problem. However, in some cases failure of self-control does have severe implications: the inability to control one's sexual desires may lead the person to commit an indecent assault or rape, failing to inhibit aggressive impulses may result in violence and so on. Given the negative, sometimes even harmful consequences of selfcontrol failure, an important question is why people often fail to inhibit their responses and give in to their urges and desires.

According to the so-called strength model, the answer to this question is that selfcontrol is costly (Baumeister, Bratslavsky, Muraven, \& Tice, 1998). In this view, control- 
ling oneself consumes energy or strength of some central, limited resource. After an act of self-control, the resource becomes temporarily depleted leaving the individual in a state of ego depletion. Therefore, performance on a subsequent self-control act is impaired because of lack of resources. A wide range of studies using divergent manipulations of self-control, such as thought suppression, emotion regulation, impulse control, deliberate decision-making and memory tasks have indeed shown that when people exert self-control repeatedly within a relatively short period of time, performance on a subsequent act of self-control is likely to decrease.

The literature on ego depletion ascribes a number of features to the concept of energy resource. First, the resource is non-specific and used for a broad variety of self-control operations such as overriding or inhibiting impulses, regulating emotions, making choices and so on. Second, the resource is limited, that is, the energy required to resist a cigarette is no longer available to suppress sexual urges. This implies that control of a particular behaviour has a negative influence on future control attempts, even if these future control attempts involve different kinds of behaviour. In other words, the reason why a person commits an impulsive act may be determined by the relatively large amount of self-control that the person has exerted prior to the impulsive act. When investigating the possible causes for the commitment of an impulsive act, one should take the amount of self-control that has been exerted prior to the impulsive act into account, realizing that even at first sight meaningless control attempts can have a serious impact on the eventual failure of self-control. In line with this explanation, occurrence of crime appears to be more likely late in the evening or night. (Baumeister, Heatherton \& Tice, 1994). Gottfredson and Hirschi noticed that crimes (in particular crimes involving personal violence) tend to occur disproportionately between 1:00 and 2:00 A.M. (one exception to this pattern is burglary; about half of all burglaries take place during the day, because employed people leave their homes during the day, thereby making daytime the best opportunity for burglars). In other words, repeated control attempts during daytime may cause depletion of regulatory resources, making breakdown of self-control more likely in the late evening or night. Third, energy is similar to a muscle. Like a muscle, self-control becomes tired after use and needs rest to recover.

In one of their first studies on the ego depletion phenomenon, Baumeister, Muraven, Bratslavski and Tice (1998) confronted hungry participants with two displays of food: a stack of fresh chocolate cookies and a bowl of radishes. Next, one group of participants was instructed not to eat from the cookies but only from the radishes (high self-control), while the other group was instructed to eat only from the cook- 
ies (low self-control). In this way, participants' amount of self-control was manipulated; participants in the radish condition had to control their desire to eat from the deliciously smelling cookies. Subsequently, all participants were asked to complete a 'problem-solving task' that consisted of a series of geometrical figure-tracing puzzles. In fact, these puzzles were unsolvable and time spent on this frustrating task served as a measure of self-control performance. A group of control participants did not take part in the food part of the experiment but started directly with the puzzle task. It was found that participants who exerted self-control by resisting the tempting chocolate cookies, spent significantly less time at solving the puzzles than participants who were allowed to eat chocolate cookies and control participants who were neither hungry nor exposed to food. In other words, participants who exerted a high amount of self-control, performed relatively worse on a subsequent self-control task.

Today, the phenomenon of ego depletion is a well researched phenomenon and empirical tests have confirmed that self-regulatory resources underlie a wide range of domains. For instance, Muraven, Collins, and Nienhaus (2002) manipulated initial self-control by instructing half of their participants to suppress a forbidden thought (high self- control), whereas the other half worked on simple arithmetic problems (low self-control). Next, all participants participated in a 20 minute beer drinking session in which they were asked to rate the qualities of different beers. Although participants were allowed to drink as much beer as they liked, they were also provided a reason to reduce their alcohol intake, namely performing well on a subsequent driving test. In spite of the prospect of the driving test, participants who suppressed a forbidden thought drank significantly more beer than participants who exerted low initial selfcontrol.

Other demonstrations of ego depletion are related to emotion regulation and illustrate that participants who suppressed or exaggerated their feelings performed less well on a subsequent self-control task than participants who were allowed to act naturally (Muraven, Tice, \& Baumeister, 1998). In addition, initial depletion has been proven to affect consumer behaviour in the sense that ego depleted individuals are more likely to engage in impulse purchases (i.e., buying items that one did not intended to buy) than non-depleted individuals (Baumeister, 2002; Faber \& Vohs, 2004).

In sum, the results of these studies support the assumptions of the strength model and illustrate that after repeated self-control, ego depletion emerges and self-control performance decreases, raising the probability of impulsive (criminal) acting. 


\section{Improvement of self-control}

In order to prevent people from committing impulsive crimes or similar kinds of selfcontrol related deviant behaviour it is important to explore ways to improve self-control abilities. In many cases, especially cases involving people who possess a higher propensity to engage in criminal behaviour, the benefits of improved self-control abilities will not only appear on an individual level, but are likely to be reflected in society as well. Unfortunately, however, the implications of the limited strength model can be considered as relatively pessimistic. The model proposes that controlling the self consumes energy, causing an almost inevitable loss of self-control. Although the theory states that improvement of self-control can be established by means of training or repeated exposure, it is assumed that there is not much more one can do against the loss of energy. Fortunately, findings of recent studies favor a more positive view towards the improvement of self-control by demonstrating that the concept of energy is likely to be more complicated and dynamic than initially suggested. These findings implicate that the success or failure of self-control not only depends on available energy, but can be influenced by wide range of factors. They show that ego depletion can be counteracted by various means, which will be discussed in more detail below.

\section{External focus of attention: Focusing away from the self-control de- manding action}

According to Gottfredson and Hirschi (1990), most crimes are easy to commit and require little skill. Crime offers immediate gratification (e.g., violence to the victim, money, and sex), rather than deferred rewards. To date, numerous psychological studies have addressed the issue of immediate versus delayed gratification. Many of these studies involved research on self-control in children, and made use of the so-called delay-of-gratification paradigm (Mischel, 1974; Mischel \& Ebbesen, 1970). This paradigm captures the dilemma of choosing between an immediate, small award or a greater, delayed award. Children are presented with food that they desire (candy). The experimenter instructs the children that they will be left alone in the room. The instructions that follow pose the children to the dilemma. The child has to choose between waiting for the experimenter to return and to get two or more pieces of the desired candy as a reward, or he or she can hit a bell and the experimenter will return immediately, but in this case only one piece of candy will be given. Although children try to wait for the return of the experimenter, soon the delay becomes very trying and temptation to ring 
the bell will be experienced. An important finding in these studies was that some children were noticeably better able to delay gratification than others. It appeared that the success of these children was due to the usage of control strategies: they shifted their attention to objects in the room, or dissociated themselves by focusing on the abstract properties of desired stimuli ("thinking about candy as little clouds, or imagining that they were just a picture and not real"). In other words, when attention was focused away from the control demanding action, self-control performance improved.

In a study by Alberts et al. (2006), the influence of focus of attention on self-control performance was investigated more directly. The aim of the study was to test the effect of an external versus self-focus on physical self-control performance, namely lifting a $1.5 \mathrm{~kg}$ weight as long as possible. Physical exercise tasks, such as lifting a weight, have been found to be a sensitive measure of self-control in prior research (see for example Muraven, Tice, \& Baumeister, 1998, Study 1; Ciarocco, Sommer, \& Baumeister, 2001, Study 2). Because lifting a weight becomes fatiguing and even a little painful after a short period of time, the person feels the urge to stop lifting. Overcoming this fatigue and overriding the urge to quit, requires self-control. The physical self-control task was performed twice. In order to control for interpersonal differences, the first measurement was taken at the beginning of the experiment and served as a baseline of weight lift strength, against which the second, relevant measurement was compared. During the second performance on the physical task, participants in the internal focus condition were instructed to fully concentrate themselves on their arm and hand muscles that are involved when lifting a weight. To illustrate this, an anatomical illustration of an arm and hand was shown and the experimenter pointed which muscles and tendons are used to lift a weight. Participants in the external focus condition performed a difficult cognitive calculation task during the second weight-lifting task. The high complexity of this task leaves less capacity for other thoughts about the physical selfcontrol. The results showed that the performance on the second physical self-control task remained stable when attention was focused on an external stimulus (calculation task). When participants focused their attention on an internal stimulus (muscles), performance on the physical self-control task decreased significantly.

In sum, the above discussed findings implicate that self-control performance can be improved by using attention related control strategies. When attention is focused away from a control demanding action, self-control performance has been found to improve. In contrast, when attention is focused internally, on the control demanding situation and the feelings one experiences during this condition, self-control performance 
is expected to decrease. A positive aspect with regard to self-control improvement is that people can (often easily) be taught to learn and apply strategies. In addition, even when people posses relatively low self-control abilities, using control-strategies can still be regarded as an option for self-control improvement.

\section{Internal Focus of attention: Self-awareness}

Self-awareness can be described as a state in which one is aware of oneself as an object (Duval \& Wicklund, 1972). Looking in a mirror and speaking in front of an audience are both examples of situations in which people may become highly self-aware. Being self-aware makes sure individuals come to know their own emotions and other internal states, partially by inferring them from observation. Self-awareness is closely linked to self-control performance and engagement in criminal behaviour: loss of self-awareness contributes to failure of self-control. Under conditions of low self-awareness, people loose the capacity to think about themselves and evaluate themselves. Moreover, people become less able to consider the implications of current events for the future and seem less capable of comparing themselves to appropriate standards. When selfawareness decreases, people start focusing on immediate sensations, movement and events. This attention narrowing favors giving in to temptation and has been proven to make urge driven behaviour, like violent offending, significantly more likely to occur (Nagin \& Pogarsky, 2004). In sum, when people lose self-awareness, they lose the capacity to monitor themselves. They stop to attend to what they are doing and do fail to evaluate their actions against appropriate standards (i.e. non-criminal behaviour). As a result, ordinary restraints and inhibitions are suspended and people are more likely to perform dangerous and violent acts that they would normally not engage in.

The negative consequences of low self-awareness are well reflected in the acts that often accompany the consumption of alcohol. Alcohol consumption has been shown to reduce self-awareness (Hull, 1981). It is well known that under the influence of alcohol people are more likely to engage in violent and aggressive acts and do things that they often regret afterwards. Moreover, the emergence of these aggressive and violent acts is not because alcohol increases or evokes them, but because alcohol lowers selfawareness and consequently reduces inhibitions against these acts (Steele \& Southwick, 1985). Another illustration of the consequences of reduced self-awareness comes from research by Mullen (1986). In this study, a large sample of incidents involving lynch mobs was analysed in order to find out whether the violence committed by lynch 
mobs could be accounted for in terms of self-attention processes. More specifically, the study focused on the relation between ratio of mob members to victims and atrocity (hanging, shooting, burning etc.) in lynch mobs. The results showed a positive relation between the number of lynchers relative to number of victims and the viciousness with which the victims were killed. In other words, the higher the number of lynchers, the more violence. It was suggested that as the lynchers became more numerous relative to the victims, the lynchers became less self-aware (more de-individuated), causing a breakdown of self-control, which in turn led to an increase in violent behaviours.

Recent findings on self-control and self-awareness suggest that enhancement of self-awareness may help to improve self-control performance and overcome the effects of ego depletion. In a study by Alberts, Martijn, and de Vries (2006) participants were initially depleted by means of an attention self-control task. This 8 minute task required participants to calculate and name the sum of digits presented on a computer screen. During the task, they were auditively distracted by a voice randomly naming digits and thereby interfering with their calculations. Thus, participants had to focus their attention on the calculations on the screen and try to override their impulse to listen to the interfering noise. A control group performed the same calculations, but was not exposed to the interfering noise. Next, participants were asked to complete a "verbal ability task". In fact, this was a Scrambled Sentence Task (SST) that intended to activate high self-awareness in participants. Participants in the high self-awareness unscrambled 25 scrambled sentences which were related to the self (e.g., "I read books"); Participants in the low self-awareness condition received 25 neutral scrambled sentences. Depleted participants who were highly self-aware outperformed depleted low self-aware participants on a physical self-control measurement. When self-awareness of participants was increased by means of the scrambled sentence task, participants did not show any sign of ego depletion and were able to perform relatively well.

Obviously, attentional processes can be considered as important moderators of self-control performance. It was already demonstrated that when people focus their attention externally, away from a control demanding situation, self-control performance increased. In contrast, when attention was directed towards the control demanding situation, self-control performance decreased. In addition, the current paragraph stressed that self-control performance can also be enhanced by making people aware of themselves. Although self-awareness and focusing on a control demanding situation may both be considered as forms of internal attention, they are not the same. When people focus on a control demanding action, they are applying a specific focus. 
In this case, people are primarily dealing with the experience of exerting self-control and the unpleasantness of this experience. On the other hand, when people become self-aware, they apply a more general focus on the self. As a consequence, evaluative processes are expected to be initiated and people start to compare themselves to appropriate standards. This evaluative aspect that accompanies heightened self-awareness has repeatedly been proven to result in improved self-control performance.

\section{Self-control related expectancies}

As discussed before, the strength model stresses the limits of self-control by suggesting that a limited resource underlies self-control performance. An important question with regard to this suggestion concerns the subjectivity of these limits. Does it matter whether people think of their resources or self-control capacities as limited or unlimited? In other words, do expectations and beliefs about the limits of one's resources influence self-control performance? Recent studies have addresses this issue and investigated whether expectations or implicit beliefs about the nature of self-control affect people's self-control performance. In a study by Martijn et al. (2002), it was demonstrated that the notion of limited energy is relative and flexible and that the perceived boundaries of one's self-controlling abilities are apt to change. In that study, participants watched a highly disgusting video fragment and were asked to suppress their emotions. A control group watched the same video, but received no suppression instructions. Immediately after, half of the participants who suppressed their emotions were informed that "often people think they have to rest after an effortful task. However, scientific investigations prove that this is not the case after emotional effort..." (Martijn et al., 2002; p. 446, Experiment 1). On a subsequent self-control demanding task, participants who received this information outperformed control participants and participants who received no such information. These results suggest that people expect that exercising self-control leads to fatigue and, hence to worse performance on a second self-control demanding task. If such expectancy is challenged, decline in performance is less self-evident and people may even achieve better.

In addition, in another study by Martijn et al. (2002, Experiment 2) some evidence was found that people distinguish between two classes of beliefs about exercising selfcontrol. The first class of beliefs characterizes self-control as energy and comes close to the strength model of Baumeister and colleagues. Thus, people expect and believe that they have only a limited amount of energy available for their self-control operations 
and that they are likely to fail when demands are too high. Furthermore, for optimal performance one needs to be well-rested and free of other self-control demands. At the same time, albeit to a lesser extent, people adhere to the belief that self-control is primarily a matter of motivation: if you really want to, you can do it and if you really make an effort you can do much more than you think. The distinction between these two classes of beliefs about self-control was recently corroborated by a comparable classification by Mukhopadhyay and Johar (2005) who showed that participants who think that self-control is an unlimited and malleable ability tend to set more goals.

The influence of beliefs and expectancies on self-regulatory efforts was further demonstrated in a study by Bushman, Baumeister, and Philips (2001). This study tested the hypothesis that people often exhibit aggressive behaviour because they expect that aggression will enable them to feel better. In other words, aggression may be caused by the expectancy that negative affect can be regulated by acting aggressively. This implicates that if people believe that their affective state will not be improved by aggressing, they will not aggress. In order to test this hypothesis, participants were given a bogus mood-freezing pill. They were led to believe that this pill temporarily "freezes" emotional states. As a consequence, emotional states cannot be changed, thereby making affect regulation efforts ineffective. The results of this study showed that participants who had been induced to accept the value of expressing aggression as true, responded significantly more aggressively than control participants to an offensive. However, feelings of aggression were eliminated by the mood-freezing pill. Thus, aggressive feelings depended on the expectation that their negative mood (resulting from an offense), could be changed by acting aggressively. When this expectation was removed by the mood-freezing pill manipulation, aggression decreased.

The research findings presented in this paragraph stress the importance and implications of expectancies. These findings indicate that self-control performance and ego depletion are highly susceptible to how people think self-control operates. Self-control performance was found to benefit from positive self-control related expectancies. Rather than limitedness and failure, positive control expectancies are characterized by motivation and the accomplishment of goals. In the light of these findings, it seems reasonable to assume that positive control expectancies can help to control the impulses that underlie many forms of criminal behaviour. 


\section{Motivation}

Muraven and Slessarava (2003) conducted a series of experiments in which they demonstrated that ego depletion may be compensated by increasing people's motivation to perform well on subsequent self-control task. They showed that initially depleted individuals persevered longer at a subsequent self-control task if they were led to believe that their persistence would be beneficial to others or to themselves. Depleted participants, who were not motivated, lowered their persistence and thus showed ego depletion. Thus, by increasing the motivation to exert self-control, self-control performance may be enhanced.

\section{Guidelines}

The research findings discussed in the previous section highlighted the changeable nature of self-control and provided optimistic and practicable perspectives for improving self-control performance and prevention of impulsive acting. These findings are particularly useful for improving the likeliness of taking the right form of action and can be translated into practical guidelines. Concrete guidelines for better self-control are presented in the paragraphs below.

\section{Training}

Like muscles profit from a balanced mix of training and recovery, self-control may also be strengthened by a regime of training and rest. Some evidence for this was provided by Muraven, Baumeister and Tice (1999), who instructed students to engage in selfcontrol trainings (such as keeping food diaries or improving posture) for a 2-week period. After that period, students showed improved performance on a physical selfcontrol measurement when compared to a control group. In line with this, a study conducted by Oaten and Cheng (2006) revealed that a study intervention program, a form of self-control training, improved regulatory performance. Students who participated in this study program were asked to repeatedly perform various acts of self-control, such as self-monitoring and creating artificial early deadlines, during two months. After these two months, they reported no increased stress during exam time and significant improvements in many self-control related behaviours. These students not only studied more and enhanced their study habits; they also displayed improvement of regulatory behaviour outside the context of academic habits compared to those who 
did not participate in the study program. For instance, experimental group showed a decrease in alcohol, tobacco and caffeine consumption. Also, an increase in healthy dietary habits and emotional control were observed. Additional findings on laboratory tasks suggested that the program helped to reduce (but not eliminate) the effects of ego depletion: the deterioration of performance after completing a self-control task was significantly greater for control participants than for participants who were exposed to the study program.

Although the above described findings consistently show that training can help to increase self-control abilities, it is important to take the difficulty of the self-control related goal into account. According to Baumeister and Exline (2000), some self-control tasks may be highly depleting. For instance, ex-detainees who attempt to start up an honest life and keep to the straight and narrow may find it extremely difficult to give up their delinquent habits that may have become routine for many years. It is assumed that the control of these routine behaviours can pose a major challenge for the energyresource. The authors state that "they might represent a constant drain on the reservoir, a marathon-like demand on the "muscle" (Baumeister \& Exline, 2000, p. 36). They propose that in these cases, improvement of self-control is best established by gradual increases of self-control instead of sudden ones. However, many criminal and analogous behaviours demand rigid and immediate adherence, such as the inhibition of violent impulses. In these cases, it is suggested that a "one-day at a time" strategy may be the best option. If people can consider the self-control challenge as being only for the current day (or hour or minute), they may be able to exert the amount of selfcontrol required to prevent failure. It is suggested that social support plays an essential role in such situations, namely by offering positive modeling as well as by increasing social pressure in favor of self-control related behaviour. These functions can make the desired action automatic, which will decrease the amount of self-control needed.

\section{Increasing self-awareness}

Self-awareness can be enhanced by several means. A common way to enhance selfawareness in an experimental setting is by means of a mirror or cameras (e.g. Buss, 1980; Baldwin \& Holmes, 1987). The effect of cameras and mirrors was well demonstrated in a study by Bailey, Leonard, Cranston and Taylor (1983). In their study, the effect of self-awareness on the aggressive behaviour of intoxicated individuals was investigated. Participants competed in a task in which they could hurt and/or be hurt 
by their opponent. In the high self-awareness condition, participants competed in the presence of a mirror and video camera. In the low self-awareness condition, no mirror or video camera was present. Results showed that intoxicated and non-intoxicated participants in the high self-awareness condition were significantly less aggressive than those in the low-self-awareness condition. Moreover, the finding that intoxicated participants were just as responsive to the camera and mirror as the sober participants, even under conditions of increasing provocation, illustrates the potential power and effectiveness of these manipulations. Therefore, these manipulations may well be used outside the laboratory. For instance, in order to reduce violence at nightclubs or discotheques cameras could be installed at positions were aggression is most likely to take place (e.g., near the exit of the building). When doing so, one should make sure that people are aware that they are being monitored, for instance by placing signs on the wall indicating the presence of cameras.

Interventions like cameras and mirrors concern temporary and momentary changes in self-awareness. In contrast to these short-lived changes of self-awareness, selfawareness may also be enhanced in a more long term context, by gaining an overall increased sense of self-awareness. Self-regulatory goals are more likely to be accomplished when people become aware of their own abilities, interests, limitations, biases and so on. Crime (relapse) prevention programs that aim at preventing offenders (or non-offenders) from committing crimes are often contributing to an overall increased sense of self-awareness. An example of such a program was the so-called "Operation Kick It". This project recruited imprisoned drug offenders to describe their criminal histories and the consequences in order to prevent young people and delinquents from committing crimes. In Scott, Hawkins, and Farnsworth (1994) the success of "Operation Kick It" was investigated. It was shown that only 20 percent of released prisoners who had taken part in Operation Kick-It relapsed and returned to prison. Among the matched ex-prisoners of the control-group (who did not participate in the program), the recidivism rate was significantly higher, namely 66 percent. The success of the program was partly attributed to the establishment of increased self-awareness among the participants. During the program, participants openly and honestly confronted and judged their own behaviour. Self-awareness was also enhanced in presentations that honestly disclosed past mistakes that led to lawbreaking and imprisonment to a public forum. Moreover, the program stressed the acceptance of the view that past behaviour of participants was wrongful by society's standards. The alteration of standards is essential with regard to self-awareness, since increased self-awareness encourages 
individuals to compare themselves to available standards and norms. Obviously, selfcontrol enhancing effect of increased self-awareness will only serve its purpose when the available standards concern appropriate behaviour.

\section{Changing expectancies}

The findings on the link between expectancies and self-control, as discussed in the previous section, show the influence of people's beliefs on self-control efforts and performance. First, findings of a study by Bushman and colleagues (2001) suggested that aggressive behaviour often occurs because people expect that by engaging in aggression, they will get rid of their anger and feel better. When this expectation was challenged or absent, aggressive responses were significantly reduced. This finding not only illustrates the influence and importance of beliefs, it also implicates that by changing aggression related beliefs, the harm that accompanies aggression can be reduced. Second, it was found that positive control expectancies can help to overcome the control inhibiting effects of ego depletion, making successful self-control more likely (Martijn et al., 2002). Positive control expectancies are closely related to the concept of self-efficacy (Bandura, 1986). Self-efficacy is defined as "people's judgments of their capabilities to organize and execute courses of action required to attain designated types of performance" (Bandura, 1986, p. 391). In other words, self-efficacy refers to the extent that people believe they can successfully carry out the behaviour required to produce a certain outcome. It is efficacy expectations that affect both the initiation and perseverance of coping behaviour. As shown by recent studies, some people believe that they do not (or to lesser extent) possess the capacity or resources to successfully deal with the urges and desires they experience (Martijn et al., 2002). Since individuals' perceptions of self-efficacy can influence their choice of activities and settings, and can determine how much effort they will invest when facing obstacles, these people are more likely to experience difficulties when trying to overcome the immediate gratification offered by impulsive crimes.

Self-efficacy theory provides a useful framework for treatment programs and interventions tailored to deviant (criminal) behaviour. Self-efficacy can be modified through learning experiences such as task mastery and vicarious learning (Bandura, 1986). For instance, a concrete way to improve positive control beliefs is by exposure to self-control activities that become incrementally more challenging. By mastering these activities, people not only achieve mastery over their immediate environment, 
they also develop the self-efficacy that is assumed to transfer this mastery to daily life.

A more passive learning method to enhance feelings of self-efficacy is by means of verbal persuasion; telling one that he or she can competently perform a particular behaviour (Bandura, 1986). Verbal persuasion may entail a "pep talk" or specific performance feedback and may provide a "boost" to counter occasional obstacles. Being told by others that one can competently perform a particular behaviour can lead to increased self-efficacy and improvement of self-control. In line with this, findings of a study by Martijn, Alberts and Goedegebuure (2006) revealed that when people were led to believe that they possessed good self-control abilities, their self-control performance improved significantly. When participants exerted self-control repeatedly and received positive feedback on their self-control performance, performance on a subsequent self-control task remained relatively stable and no signs of ego depletion were present.

Since verbal persuasion is easy to provide and generally available (Liebert \& Spiegler, 1994), relapse preventions may well benefit from implementing programs in which people are helped to believe that they are capable of resisting the urge to commit (impulsive) crimes and able to keep to the straight and narrow. Although verbal persuasion has its limits, under the proper conditions it can be very effective (see Bandura, 1986 for an overview of these conditions). In combination with more active learning experiences, verbal persuasion techniques may help to increase self-efficacy expectation, positively contributing to the success of future self-control attempts.

\section{Using attention strategies}

Whether people attend to immediate rewards offered by (impulsive) crimes or are capable of avoiding the immediate reward is often a crucial factor. In order to overcome the immediate pleasures and outcomes associated with criminal behaviour, research has suggested that strategies can help to delay gratification and successfully control oneself. It has been shown that the more people think about the instant pleasure, the harder it becomes to resist it (Karniol \& Miller, 1983). There a several strategies that can help people to focus their attention away from the immediate stimuli that trigger impulses to engage in a behaviour, making successful self-control more likely. Among these are self-instructions, focusing on the better reward (imagining the temporally remote goal) and learning to distract oneself with other thoughts of behaviour. However, attention strategies are not necessarily restricted to distractive cognitions. Research 
by Alberts, Martijn, Nievelstein, Jansen, and de Vries (2006) demonstrated that selfcontrol failure can also be overcome when engaging in distractive activities. Preferably, in order to successfully attract attention away from the self-control demanding situation, these activities should not directly be related to the self-control task at hand and require a considerable amount of concentration. These strategies can be learned and internalized by repeated practice and may be implemented in educational programs aimed at improving self-control abilities.

\section{Discussion}

The present chapter attempted to provide more insight in the causes of and processes underlying impulsive criminal behaviour. More specifically, an overview of psychological findings and theories was presented in order to gain more knowledge on why and under which conditions impulsive crimes are likely to occur. Instead of focusing on stable, personality related factors, the current article mainly addressed a state oriented approach, by identifying factors that influence the momentary occurrence of the impulsive (criminal) act.

Self-control was introduced as an essential psychological construct that underlies impulsive behaviour. It was argued that the commitment of an impulsive crime reflects the inability to temporarily control the self. Recent findings on self-control offers a reason why people fail to control themselves and commit impulsive acts. According to the strength model, controlling the self is psychologically demanding and results in a state of so-called ego depletion. When people control themselves, they use an amount of energy that is not available anymore for future self-control tasks, resulting in decreased performance. Although a considerable number of studies have indeed found support for this assumption, recent studies have also shown that successful self-control is not dependent on energy or previous control attempts alone and that the emergence of ego depletion can be overcome. Several studies implicate that under certain conditions, people can control themselves successfully. These conditions include: focusing attention away from a control demanding action, increased self-awareness, and presence of positive control expectancies. Instead of external control attempts, like imprisonment and introduction of severe punishments, these findings offer an alternative way for preventing criminal behaviour, namely by focusing on improvement of internal control. In the present article we provided some concrete guidelines that may help to improve self-control performance and inhibition of (destructive) impulses. These 
guidelines may be implemented in treatment programs and interventions tailored to deviant (criminal) behaviour. Moreover, the overall complexity of these guidelines is limited, making them also suitable for more auto-didactic application. Beside the use of these guidelines, a better understanding of how self-control operates, for instance by making people aware of ego depletion and its consequences, can help to improve impulse inhibition as well. Future research should investigate the impact of the suggested guidelines on impulsive (criminal) behaviour more directly, for instance by conducting longitudinal studies in which delinquents are exposed to treatment programs that are based on self-control related findings as presented in the current paper.

For the sake of generalisation, in the current chapter we mainly concentrated on the issue of momentary self-control failure (state self-control) and paid relatively little attention to the role of individual differences in self-control abilities (trait self-control). Obviously, people do differ in their self-control abilities; some people are noticeably better than others in remaining patient, saving money, regulating emotions and so on. Moreover, people may posses relatively good skills in one domain of self-control and few skills in another domain. In order to prevent impulsivity and understand when and why impulsive crimes occur, the role of individual differences should not be neglected. Optimization of treatment programs requires knowledge of the self-control skills an individual possesses, but also of other factors related to self-control performance, such as the expectancies that people hold about the nature of self-control.

Many of the causes of criminal behaviour, such as dysfunctional family conditions, lack of educational opportunities, and poverty are often difficult to alter. However, the research findings and guidelines presented in the current chapter favor a more positive viewpoint and suggest that (impulsive) criminal behaviour can be reduced by focusing on ways to enhance momentary self-control. In addition, by gaining more insight in the psychological processes that underlie impulsive crimes, it becomes clear that dealing with problems associated with criminal behaviour is not necessarily restricted to the usage of external control measures. Acknowledging that situational factors and psychological control processes play an important role in the realization of criminality, leads to the awareness that people not always deliberately choose for giving in to impulses and desires. The difference between not willing and not always being able to control oneself is crucial here; preventing deviant behaviour of those who are simply not willing to inhibit their impulses and desires may primarily be accomplished by means of external control measures. In contrast, those whose deviant behaviour is caused by an inability and lack of knowledge how to successfully control oneself, are 
also expected to benefit from internal control measures that are aimed at long-term improvement of self-control related skills. 


\section{CHAPTER 7 \\ General Discussion}


A topic that gained a considerable amount of attention by ancient philosophers like Socrates and Plato was that of so-called "akratic" action. An akratic action refers to an action that is done despite the fact that the agent considers a different course of action preferable. Many modern philosophers and psychologist have used terms such as "weakness of will, "moral weakness" and "lack of self-control", to describe this phenomenon (Elster,1979). Indeed, the question why people often fail to inhibit their responses and yield to temptation, despite the negative, sometimes even harmful consequences of this failure is not only theoretically, but also practically interesting. According to the strength model (Muraven, Tice, \& Baumeister, 1998; Muraven \& Baumeister, 2000), akratic actions emerge as a consequence of limited energy. In this model, self-control draws on a general source of energy and people are assumed to have a limited amount of energy available. After an act of self-control, the resource becomes depleted and an individual finds him- or herself in a state of ego depletion. Consequently, a second act of self-control results in impaired performance. Although the strength model seems intuitively plausible, it does not devote much attention to the possible processes of the mechanisms involved. As a result, the precise nature of ego depletion and processes that underlie failure of self-control remain unknown. The aim of the present dissertation was to gain more insight in these processes. More specifically, it was argued that by identifying distinct factors that influence (effective) self-control, more insight can be obtained in the processes that underlie self-control and potential avenues for improvement can be created. In this concluding Chapter, the main findings of the research presented in this dissertation will be discussed. In addition, suggestions for improvement of self-control and future research will be formulated.

\section{Automaticity}

Chapter 3 addressed the role of automatic processes in self-control and ego depletion. More specifically, two studies were conducted in order to investigate whether ego depletion can be reduced or even eliminated by means of manipulations that operate at an automatic level. Two different priming manipulations were used to unconsciously activate of the concept of persistence. The results of both studies consistently show that activation of persistence can have robust effects on self-control performance. Depleted participants who were primed with persistence outperformed depleted participants who were exposed to a neutral prime. Depleted participants who were exposed to the neutral prime displayed the standard ego depletion pattern: their performance de- 
creased significantly. Whereas the strength model regards self-control as a conscious, active process, these findings illustrate that self-control may also be guided by processes that operate outside awareness. The impact of these automatic processes was further demonstrated by their potential to circumvent the occurrence of ego depletion. Interestingly, a first attempt to investigate the possible downside of unconscious activation revealed that its control enhancing effect is unlikely to be followed by any kind of rebound effect; participants who kept their self-control performance stable due to the priming manipulation did not exhibit any tendency to reduce their self-control efforts later on. Note, however, that the findings of Chapter 3 do not necessarily contradict to the strength model. Since the strength model regards ego depletion as a consequence of active (conscious) self-control, it is possible that nonconscious self-control, because it does not concern active involvement by the self, will not drain regulatory resources. Although the findings of Chapter 3 do not necessarily conflict with the strength model, they do illustrate a possible limitation of this model. After all, the limited strength model is only concerned with conscious self-regulation and does not devote any attention to its unconscious counterpart. The present findings show the importance and impact of automatic self-control and suggest that the strength model may well benefit from incorporating both types of self-regulation. Interesting in this respect are socalled dual-process models (see Chaiken \& Trope, 1999; Fazio, 1990). Such models have been developed and applied in the field of cognitive and social psychology and are characterized by a differentiation between conscious and automatic processes working either in conjunction or opposition.

The findings presented in Chapter 3 are also important since they provide further empirical foundation for a direct link between unconscious processes and self-control. Previous research by Bargh and colleagues (Bargh, 1990; Bargh, Gollwitzer, Lee-Chai, Barndollar, \& Troschel, 2001) already revealed that goal pursuit can take place without any conscious guidance or intervention. It was found that goals can be triggered outside awareness and that, once activated, these goals function in the same way as consciously chosen goals (see also Gollwitzer, 1999; Gollwitzer \& Brandstätter, 1997). In a similar vein, the findings of Chapter 3 illustrate the impact of automatic processes and provide further support for the notion that self-control does not always require conscious awareness to be effective. Moreover, whereas previously discussed studies on automatic goal pursuit mainly addressed unconscious activation of self-regulatory goals, the results presented in Chapter 3 extend this body of research by showing that self-control may also be automated by unconsciously activating a behavioural con- 
struct rather than a (specific) goal.

An intriguing question in the light of the findings of Chapter 3 concerns the impact of automatic processes in our daily life. In line with Bargh's and Chartrand's claim that "... most of daily life is driven by automatic, nonconscious mental processes" (1999, p. 464), the two studies presented in Chapter 3 show that relatively subtle manipulations can have a significant effect on regulatory performance, without people being aware of this influence. Likewise, in everyday life, self-control attempts are likely to be influenced by unconscious processes set into motion directly by one's environment, like billboards, conversations, television and so on. Although people may not be directly aware of these processes, they can become aware that these processes exist and that they can have a serious impact on self-control performance. Consequently, this knowledge may be used to prevent oneself (if possible) of exposure to, or deal more effectively with "daily life manipulations" that are likely to have a detrimental effect on ones regulatory abilities. For instance, similar to the screensaver manipulation in Chapter 3, candy commercials on television may unconsciously trigger the desire to eat palatable food and may cause a dieter to get up from the couch to get some chocolate, thereby breaking his or her diet (see e.g., Papies, Stroebe, \& Aarts, 2006).

\section{Attentional focus}

Research presented in Chapter 4 and Chapter 5 investigated the role of attentional processes in self-control. In Chapter 4, a distinction was made between directing attention towards versus away from a control demanding action (internal focus or cognitive association versus external focus). In order to increase awareness of regulatory efforts, participants were asked to pay attention to their arm and hand muscles that were involved while they were performing a physical self-control task (internal focus). An external focus was induced by a distracting calculation task that was completed by participants while they exerted self-control. It was found that participants who were distracted by this calculation while exerting physical self-control outperformed both participants who focused their attention on the physical self-control task (internal focus) as well as control participants who did not receive any focus instructions. However, no significant difference in self-control performance was found between participants in the internal focus condition and control participants. With regard to the strength model, these findings suggest that attentional processes play an important role in the emergence of ego depletion and the failure of self-control. The fact that both 
people who focused attention internally and control participants' exhibited a decline in performance, might imply that the occurrence of ego depletion is partly mediated by ineffective or suboptimal attentional processes. Directing attention to one's regulatory efforts while exerting self-control, may be a default process or natural tendency that emerges when people exert (conscious) self-control. In any event, it contributes to the emergence of ego-depletion. When this default attentional process is replaced by a process that is not preliminary concerned with regulatory efforts, no negative influence on self-control performance seems to occur. A related finding in this respect comes from Vohs and Baumeister (2004) who showed that depleted participants had more problems to direct attention away from a control demanding situation. In this study, the authors argued that it would become more difficult for would-be faithful relationship partners to direct their attention away from attractive new partners under conditions of depletion. Consistent with this expectation, it was found that, compared with nondepleted participants, depleted participants spent more time looking through a book of attractive, near-naked models. In other words, besides demonstrating a link between attention and ego-depletion, this finding also suggests that the employment of an external focus may become more difficult when regulatory resources are low.

The findings of Chapter 4 may also provide more insight in the results of Chapter 3. The success of an external focus of attention may lie in its ability to circumvent active, conscious involvement by the self, thereby taxing regulatory resources too a lesser extent. Imagine a person who quit smoking and who experiences the urge to smoke a cigarette. In this situation, calling a friend can be seen as an external focus that enables the person to directing his attention away from his craving and the self-control demanding situation. In stead of engaging in an internal conflict, the self-control operation is now handed over to the distracting activity. At this point, the need for active, conscious self-control is avoided. In other words, the phone call may function as an initiation of automated self-control. Then, the effectiveness of automatic self-control may also partly be determined by the fact that there is no awareness of the effort that is involved and that the self is not posed to an internal conflict.

In a broader perspective, attentional strategies may not only be beneficial for momentary self-control enactment, but may also be considered valuable in a long term context. By repetition, the employment of attentional strategies may become internalized and automatic, thereby contributing to overall improved self-control abilities. That is, effective employment of attentional strategies may be a factor that contributes to interpersonal differences in self-control. In line with this, Rosenbaum (1990) states 
that peoples' general repertoire of learned self-control skills are essential for goal accomplishment. He uses the term "learned resourcefulness" to indicate the acquired regulatory skills that help people to control their behaviour. Highly resourceful people have learned over their lifetime to use a diverse range of self-control strategies that help them to control themselves and pursue their goals in daily life (Rosenbaum, 2000; Rosenbaum \& Ben-Ari, 1985; Kennett \& Ackerman, 1995). In contrast, low resourceful people only use a limited number of self-control strategies and are more likely to abandon self-control in the eye of frustration.

Chapter 5 pursued the issue of an internal (self-) focus of attention in greater depth. Whereas the findings of Chapter 4 suggest that self-focused attention has a negative effect on regulatory performance, other studies have reported a positive effect of self-focused attention on self-control performance (see for instance Macrae, Bodenhausen, \& Milne, 1998; Carver and Scheier, 1981, 1990; Monteith, 1993). In Chapter 5, these contradictory findings were addressed by making a distinction between two types of self-focused attention, namely cognitive association and self-awareness. It was found that the type of self-focus determines the effect of self-focus on regulatory performance. Exposure to a self-awareness manipulation caused initially depleted participants to keep their physical self-control performances constant. This group outperformed both control participants as well as participants who were also exposed to the cognitive association manipulation by which attention was directed towards the self-control action. In line with the findings of Chapter 4, no improvement of self-control was observed when participants were highly self-aware but at the same time aware of internal states (cognitive association). As stated previously, the positive effect of an external focus may be partly explained in terms of automatic processes. However, instead of automatic processes, the control enhancing effect of self-awareness seems rather determined by motivational and action oriented processes. Selfawareness increases the salience of an internalized, social and/or situational standard. Consequently, people become more motivated to reach the standard. Being motivated to reach a certain goal implies a focus on the accomplishment of a future state. In other words, the main focus is on action. Note that this is in sharp contrast with a focus on ones current state or efforts, as is the case with cognitive association. Following this line of reasoning, cognitive association and ego depletion may represent an excessive focus on the present, causing people to withhold themselves from reaching a future goal. Empirical support for this rationale was provided by Vohs and Schmeichel (2003) who found that when people are depleted, time perception alters. More specifically, 
ego depletion caused participants to believe that they had been continuing an activity longer than people who did not engage in prior self-control. In other words, in a state of depletion, the present becomes extended and durations seem longer. People become caught in the present and are incapable of orienting their behaviour toward future outcomes. This insight also sheds new light on the finding that priming of goals facilitates self-regulation. Goal priming may cause people to become more action oriented and focused on the accomplishment of the primed goal. If true, then goal priming should also be capable of avoiding the excessive focus on the present and offsetting the ego depletion effect. Although this idea has not been investigated yet, it may entail an interesting avenue for future research.

\section{Expectancies}

Chapter 2 addressed the relation between expectancies about self-control and self-control abilities. Whereas the strength model assumes that self-control is dependent on energy, the research in Chapter 2 focused on the question whether it matters if people expect that self-control relies on energy. In three questionnaire studies, a moderate but consistent relation was found between people's expectancies about self-control and their self-control abilities. The more people expect self-control to be dependent on (limited) energy, the less good self-control abilities they report. In the light of these findings, it may be argued that self-control performance may be partly guided by people's expectancies about self-control. However, since the research in Chapter 2 relied solely on self-report measures, future research should further clarify the impact of such beliefs by using more behavioural self-control measurements.

The findings of Chapter 2 may also provide more insight in the underlying processes of the results presented in Chapter 3. In Chapter 3, priming the concept of persistence was found to enhance self-control performance, thereby extinguishing the ego depletion effect. When faced with a series of control-demanding operations, unconscious activation of persistence may circumvent people to act upon the implicit theory that self-control consumes energy. Activation of persistence may cause an alternative implicit theory, which is characterized by a more achievement focused vision on selfcontrol, to become more accessible. In other words, the regulatory behaviour of an individual who is primed with persistence may resemble the self-control actions of an individual who beliefs that self-control performance is mainly a matter of persistence and motivation (rather than energy). In that case, the salience of a self-control enhanc- 
ing state of mind may function as a strong determinant of the extent to which people are able to control themselves successfully.

\section{Limitations}

The present dissertation is limited in several aspects. The first limitation includes the way self-control performance was measured. Most of the research presented in this dissertation relied on a physical measure of self-control. These physical measures have been proven to be a sensitive measure of self-control in prior research (see for example Muraven, Tice, \& Baumeister, 1998, Study 1; Ciarocco, Sommer, \& Baumeister, 2001, Study 2). However, for the sake of generalisation, future research aimed at examining the present findings in more depth should also consider different ways of assessing self-control performance. Possibilities include measuring persistence in the eye of frustration (see for instance Baumeister et al., 1998) or tasks that assess the ability to postpone immediate reward (see for instance the Iowa Gambling Task; Bechara, Damasio, Damasio, \& Anderson, 1994).

A second limitation concerns the fact that the present (as well as previous) research on ego depletion relied on procedures that typically involve a sequence of two self-control tasks. In most cases, the first task aims to manipulate the level of depletion whereas the second task attempts to measure self-control performance. At this point, it remains unclear how ego depletion operates in a larger sequence of self-control tasks. This seems especially relevant given the fact that in daily life, people often engage in numerous self-control attempts. Therefore, future research should investigate self-control and the emergence of ego depletion also in a more realistic context, namely by incorporating more instances of self-control into the research design. A possible way to gain more insight in how ego depletion operates in daily life is by means of an experience sampling technique. In this technique, people describe the experience of specific moments in detail (see for instance Larson, 1995). While people engage in their normal daily routines, they carry a device that is used to signal them periodically to record their current activities, feelings and so on. In this way, research may go beyond the finding that ego depletion emerges after a temporary phase of self-control, thereby providing more insight in the causality and implications of the phenomenon.

Another possible limitation concerns the homogeneity of the research population. Most studies included a homogenous group of psychology students. Although it can be assumed that self-control involves fundamental and general processes, it would be 
interesting to apply the research design to other populations as well, in order to establish transferability. For instance, from a clinical perspective it may be interesting to investigate to what extent the manipulations used in the current dissertation can help to improve self-control performance of people who suffer from drug addiction, compulsive behaviour, eating disorders and so on.

\section{A dynamic approach of self-control}

The research presented in this dissertation has highlighted the malleable nature of selfcontrol and has shown that ego depletion is less inevitable than initially suggested. Although these findings do not necessarily contradict the strength model, they challenge the view that self-control is dependent on energy alone. Self-control may indeed depend on limits. After all, people are not able to control themselves infinitely. However, findings in the current dissertation favor a more dynamic view on self-control in which the flexibility of self-control limits are taken into account. In this section, such a dynamic vision on self-control is presented.

The vision we are about to discuss can best be regarded as an extension to the strength model. In line with the strength model, the current vision assumes that selfcontrol performance is expected to be dependent on the current amount of energy an individual has. In addition, since ego depletion seems to be influenced by several factors like expectancies, attentional strategies and automatic processes, these factors are assumed to contribute to self-control performance and the emergence of ego depletion as well. These factors are able to compensate for the emergence of ego depletion. The factors that are present in a self-control situation are labeled as compensator. A compensator is thus a generic term for one or more factors that influence(s) self-control performance and ego depletion. Each factor varies in its contribution to overall selfcontrol performance. For instance, the presence of positive self-control expectancies contributes positively to performance, whereas tempting short term goals will rather have a disadvantageous effect. The impact of the compensator is thus determined by the relative weight of the factors that are present in a given self-control demanding situation. While some factors may contribute positively to the compensator (e.g. positive control expectancies), others may lower the impact of the compensator (e.g. stress). Moreover, factors can counterbalance each other. For instance, while stress may contribute negatively to the value and impact of the compensator, a high amount of motivation at the same time may higher the value of the compensator, thereby (partly) 
covering the negative impact of stress. The higher the value of the compensator, the more positive its effect is expected to be. Note that a compensator may also contribute negatively to self-control performance. When for instance both stress and negative control expectancies are present too a large extent, this may actually inhibit self-control performance. In this case, a relatively high amount of energy is necessary for successful self-control.

In sum, the current view states that the amount of self-control a person will exert in a given situation is expected to be determined by (1) the current amount of energy a person has and (2) the value (contribution) of the compensator. In other words, whereas the limited strength model predicts that a low amount of energy will cause breakdown of self-control, the current vision states that self-control performance is also determined by the contribution of the compensator. In concrete terms, this means that when a relatively low amount of energy is present in a certain situation, a high value of the compensator may compensate for the low level of energy, thereby increasing the likelihood of successful self-control. A negative contribution of the compensator, on the other hand, requires a higher amount of energy for successful self-control.
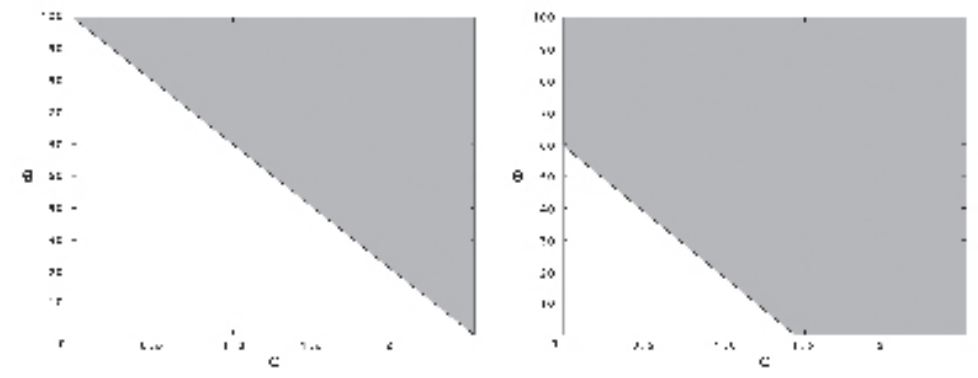

Fig. 1. Two situations in which a different amount of self-control is required. The left graph represents a situation in which more self-control is required than the right graph as indicated by the higher position of the threshold.

Fig. 1 depicts a visual representation of this view. The $\mathrm{x}$-axis represents the presence of the compensator. The position on the $\mathrm{x}$-as shows the extent to which the compensator is present. Values of the compensator smaller than 1 represent a negative effect of the compensator, whereas values greater than 1 indicate a positive effect of the compensator. A value of 1 corresponds to a situation in which neither a negative nor positive in- 
fluence of the compensator is present (neutral value). The y-axis represents the amount of energy that is present at that moment. The higher the position on the y-as, the more energy is available. Since successful self-control implies a dichotomy, namely either being successful or unsuccessful, a threshold needs to be incorporated. This threshold is represented by the line that separates the darkened area from the white area ${ }^{1}$. When a point is located in the darkened area, sufficient self-control is exerted. When a point is located in the white area below the threshold, an insufficient amount of self-control is exerted. Obviously, some situations or tasks require more self-control than others. The height of the threshold is therefore determined by the self-control requirements of a given situation. The higher the self-control demands, the higher the threshold will be. In Fig. 1, two different self-control situations are illustrated. Since the position of the threshold is higher in the left graph compared to the right graph, the left graph depicts a situation in which relatively more self-control is needed than in the right graph.

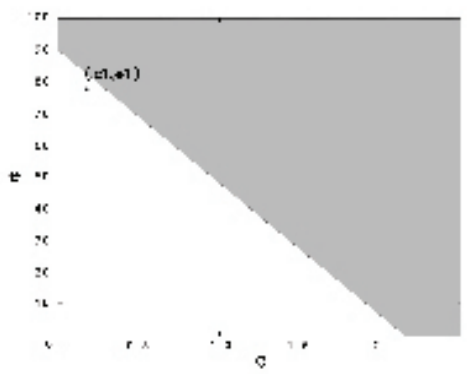

Fig. 2. A self-control demanding situation in which a relatively high level of energy and a low value of the compensator are present. Point $(\mathrm{cl}, \mathrm{el})$ is not located in the darkened area, indicating that the self-control requirements of this situation are not met.

Fig. 2 illustrates a situation that is characterized by a high level of energy and a low value (negative contribution) of the compensator. Point (c1, e1) is located below the threshold, indicating that despite the high amount of energy, an insufficient amount of self-control is exerted. An example of such a situation is the fact that students often study insufficiently during the first week(s) of a course (causing them to do much of the work in the last week). In this case, the distance of the goal of passing the exam

1 For the sake of simplicity, a straight line has been chosen to represent the threshold. However, it may be possible that in some situations the threshold for successful self-control is better represented by a non-linear curve. 
may pose a significant negative contribution to the compensator. In addition, this negative value of the compensator may further be intensified by the presence of attractive alternatives, such as spending time with friends.

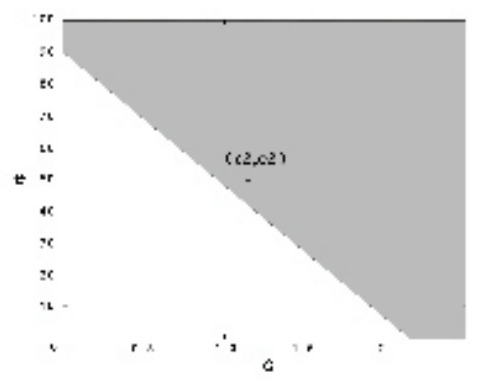

Fig. 3. A self-control demanding situation in which a moderate level of energy and a moderate value of the compensator are present. Point (c2, e2) is located in the darkened area, indicating that it is likely that sufficient self-control will be exerted.

Fig. 3 illustrates a second situation in which a moderate level of energy and a moderate value of the compensator are present. The fact that point (c2, e2) exceeds the threshold indicates a sufficient amount and of self-control and a high likeliness of successful self-regulation in this situation.

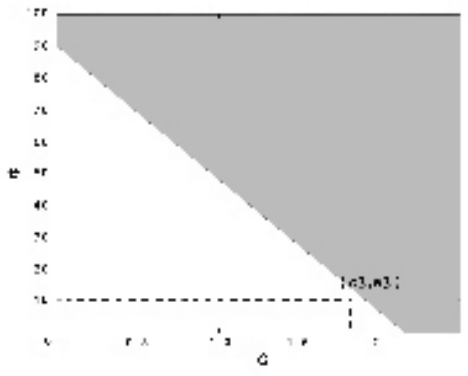

Fig. 4. A self-control demanding situation in which a low level of energy and a high value of the compensator are present. In this situation, it is unlikely that sufficient self-control will be exerted since point (c3, e3) is located outside the darkened area. 
The situation indicated by point (m3, e3) in Fig. 4 concerns a low amount of energy and a high value of the compensator. In this situation, the relatively low amount of energy entails the main cause for the high likeliness of regulatory failure. An example of this situation is a student who studies the day before the exam and notices that, after numerous hours of studying, he or she is not able to successfully exert self-control anymore. Despite the proximity and importance of the exam (high value of compensator), breakdown of self-control is the inevitable result. In this case, the compensator is incapable of compensating for the low amount of energy.

The above described view attempted to integrate the findings of the present dissertation into the strength model. Rather than rejecting the strength model, this view can be considered as a framework that extends the strength model. The increased complexity of the current vision may seem disadvantageous, but it also takes a more realistic view on self-control. After all, self-control entails complex cognitive processes that are unlikely to be explained in terms of a single metaphor (Cervone, 1996). Self-control performance is more likely to be susceptible to a wide range of constantly changing influences, both internal and external, that interact with each other. This interaction may be a fascinating area for future research. By addressing the interaction of multiple moderating factors and their relative weight, future research may shed more light on the nature and implications of this interaction, thereby gaining a clearer understanding of how self-control operates.

The above described view illustrates the value of considering self-control from a more dynamic point of view. That is, such a view can account for some findings that are anomalies for the strength analogy. For instance, according to the strength model, self-control should become stronger with repeated practice. However, this assumption does not explain relapses of self-control failure such as cigarette smokers who refrain successfully for short periods, but do not succeed in definitely quitting their habit. The addition of compensators to the concept of strength may well explain why people sometimes fail to control themselves, even when this failure is preceded by a period of self-control "training". For instance, situational factors like stress and negative effect may have a detrimental effect on the value of the compensator, thereby contributing negatively to self-control, even when the current amount of energy is relatively high (Oaten \& Cheng, 2005; Santrock, 1976). 


\section{Implications: Improvement of self-control}

Most suggestions to improve self-regulatory strength and, hence, to avoid ego depletion are primarily inspired by the 'self-control as muscle' analogy (Baumeister et al., 2000). Like muscles profit from a balanced combination of training and recovery, selfcontrol may also be strengthened by a regime of training and rest. Research has indeed found support for this notion (Muraven, Baumeister, \& Tice, 1999; Oaten \& Cheng, 2006). In addition to the training of self-control, the findings presented in the current dissertation imply that there are additional ways to improve self-control performance.

The findings of Chapter 3 illustrate the role of automatic processes and suggest that self-control may become less effortful, and thus less depleting when self-control operations are automated. A possible way to automate self-regulation is by repeated and consistent use of self-regulatory skills in response to specific cues (Kanfer, 1996). Repeated use of self-regulatory skills in reaction to specific cues enables people to develop the capability to automatically trigger ways that will facilitate self-control. Stated differently, skills that are aimed at maintaining self-control can become automatically activated by environmental cues. As pointed out in Chapter 4, an example of a selfcontrol skill may be the ability to shift attention away from a control demanding action. Imagine a dieter who is exposed to tempting food. Repeated use of attentional skills in the presence of "forbidden food" related cues may cause the dieter to associate the cues with these skills. Consequently, attentional strategies may eventually become triggered and successfully acted upon without any conscious intervention.

Another way to facilitate nonconscious or automatic self-regulation is by using socalled implementation intentions (Gollwitzer, 1999; Gollwitzer \& Brandstätter, 1997). These are action plans and refer to specific goal behaviour as well as to the situational context in which the planned behaviour will be enacted (e.g., when I encounter situation $\mathrm{x}$, I will initiate goal-directed behaviour y). The process of self-control becomes automated because behavioural control is overtaken by anticipated environmental cues. The impact of implementation intentions on self-control and ego-depletion was demonstrated by research of Webb and Sheeran (2003). In two studies, it was consistently found that forming implementation intentions helped depleted participants to perform significantly better on a subsequent self-control task compared to depleted participants who did not form implementation intentions. Moreover, in a recent series of studies by Martijn, Alberts, Sheeran, Peters, Mikolajczak, and de Vries (2007), it was found that implementation intentions lead to more determined and constant goal-striving. When confronted with an unexpected barrier, participants who formed 
implementation intentions were more tenacious, displayed a higher quality of repeated goal-directed behavior and used alternative strategies of goal-striving more frequently compared to participants who only formed a goal intention.

Interestingly, an additional reason why implementation intentions may be beneficial for self-control has to do with its conscious part, namely forming the intention. As pointed out earlier, the findings of Chapter 3 and Chapter 4 suggest that ego depletion represents an excessive focus on the present, causing people to withhold themselves from achieving a future goal. By forming implementation intentions, people make action plans and shift their focus toward a future goal, thereby reducing their focus on the present. In terms of action control theory (Kuhl, 1982), consciously choosing a goal and selecting appropriate cues may help people to become more action oriented, making successful self-control more likely.

The findings of Chapter 4 demonstrated that self-control performance can be improved when people focus their attention away from a control demanding stimulus or situation. Findings from previous research suggest that it is important to consider the way these attentional strategies are conceptualized (Wegner, 1994; Wegner et al., 1987; Wegner, Erber \& Zanakos, 1993, Wegner, Broome \& Blumberg, 1997). According to Wegner's theory of ironic processes of mental control, trying not to think about something often causes the unwanted thoughts to come back stronger than before (Wegner, 1994). Thus, the attempt to distract oneself by not thinking of a self-control demanding stimulus is, ironically, likely to result in thoughts about this stimulus. Wegner's theory poses that attempts to avoid thoughts are driven by conscious, controlled searches for other thoughts. In order to check whether the attempt is successful, unconscious, automatic searches for the unwanted thoughts are present at the same time. Under conditions of high load, the automatic search dominates over the controlled search and brings the unwanted thought back into consciousness. Fortunately, thought control is suggested to become more effective when it is conceptualized as efforts at the creation of states of mind, rather than the avoidance or suppression of states. Thus, instead of trying not to think about fattening food, it is more efficient for a dieter to think of all the tasty food that he or she is allowed to eat. In the same way, engaging in a distracting activity (see for instance Chapter 4) may facilitate the creation of a new state of mind that is not directly related to the control demanding situation. Taking Wegner's claim that "intentional concentration seems to work better than intentional suppression" (1994, p. 41) into account, distracting activities should preferably require a considerable amount of concentration. 
The findings presented in Chapter 2 suggest that self-control performance may be partly guided by people's beliefs and expectancies about how self-control operates. This may particularly be useful for interventions that concern a certain type of selfcontrol related behaviour, i.e., refraining from bad habits such as smoking or sticking to healthy habits such as regular exercise. Whereas many of these interventions already incorporated people's expectancies about their own abilities (self-efficacy) (for an overview see Hevey, Smith, \& McGee, 1998), the findings of Chapter 2 suggest that these interventions may also benefit from addressing people beliefs concerning the operation of self-control. For example, such interventions could start with an inventory and the explicit formulation of such beliefs. If necessary, inappropriate controlbeliefs may be systematically challenged and in doing so, modified for more fruitful ones. Research in the field of alcohol prevention has already shown that programs that challenge incorrect beliefs about alcohol are effective in enhancing self-regulation by reducing problem use of alcohol (see for instance Marlatt et al., 1998).

Future research may also combine the findings of Chapter 2 with the findings of Chapter 4. Previous research from Kirsch, Mearns, and Catanzaro (1990) showed that expectancies for negative mood regulation were positively correlated with the use of active, problem-oriented coping strategies and negatively associated with avoidance strategies (see also Mearns, 1991). In addition, in a study by Akgun (2004) it was found that higher self-efficacy expectancies correlated positively with high resourcefulness (overall self-control strategy use). In the same way, energy related expectancies of selfcontrol may correlate with the use of self-control strategies, such as attentional strategies.

\section{Future research}

Although the previous sections already detailed some routes for further exploration, some additional avenues of future research are proposed in the current section. The present dissertation has detailed some marked findings regarding the potential processes that underlie self-control performance. However, much of these processes remains to be specified. This may be done in ways similar to those applied in the current dissertation, namely by investigating potential factors that circumvent the occurrence of ego depletion. However, the uncovering of these processes may also be accomplished in a different way. Whereas previous research has mainly addressed the impact of ego depletion on a certain outcome behaviour, future research may also investigate the 
impact of ego depletion on cognitive functions that are known to influence self-control. Examples of such functions include goal setting (e.g. Oettingen, Pak, \& Schnetter, 2001), alternate goal shielding (Shah, Friedman, \& Kruglanski, 2002) and ruminative coping (Nolen-Hoeksema, 1998). A more detailed portrait of the link between such functions and ego depletion should add to our understanding of the nature of ego depletion and will therefore further elucidate the processes that underlie regulatory failure.

Another potential area for future research may be the relation between ego depletion and exertion. If consecutive exercise of control depletes some energy source, it seems reasonable to assume that this should be reflected in an increased fatigue. A somewhat remarkable finding in this respect is that the studies described in Chapter 3 , 4 and 5 reveal that even when objective signs of depletion are present (e.g., worse performance on physical self-control tasks) people still report no significant differences in fatigue. It is possible that the absence of differences in reported fatigue reflect the inability of people to correctly report inner states of fatigue. Autonomic process measures as indicators to detect changes in arousal and effort may circumvent this inability and may therefore be considered as a good alternative for testing the relation between ego depletion and exertion. By using autonomic measures such as skin conductance, heart rate and finger pulse volume, future research may not only shed more light on the link between ego depletion, but may also reveal to what extent subjective experience and autonomic activity match.

As mentioned before, research by Vohs and Schmeichel (2003) showed that participants who took part in a self-control demanding task, as compared to a control group, systematically overestimated the duration of that task. This belief may result in abandonment of further self-control. This points to the fact that subsequent self-control efforts are, at least in part, guided by subjective interpretations of prior efforts and not by the 'objective' amount of energy invested per se. Interpretation of prior efforts may constitute an important process that contributes to self-control failure: prior selfcontrol efforts may be used to justify ones decision to give in to impulses or desires. In other words, the fact that people become aware of their effort investment during selfcontrol may function as a "legitimate" reason to quit future self-control attempts. This implies that the occurrence of ego depletion (at least partly) reflects the interpretation of one's prior regulatory efforts. Moreover, the extent to which people involve in justification is not only likely to determine reactions to success and failure, but may influence future goal-setting and goal-striving behaviour as well. Clearly, this issue awaits future 
study and further highlights the utility of considering the role of processes in self-control performance.

\section{Concluding remarks}

Almost 10 years ago, when the ego depletion phenomenon was first demonstrated by Baumeister and colleagues, it was presented as a default reaction that was inherently linked to self-control efforts. Indeed, a large amount of studies conducted so far has proven the robustness of the finding. In fact, we were one of the first researchers that were able to replicate the phenomenon in our own laboratory. However, the findings presented in this dissertation suggest that default does not mean inevitable. The current results illustrate that people can exert self-control repeatedly without any signs of ego depletion or diminished performance. This finding has led to some new insights in the processes that underlie self-control and ego depletion. The occurrence of ego depletion seems to be linked to conscious and active self-control attempts. When selfcontrol is automated, no depletion effects seem to occur. In addition, also attentional processes seem to play a pivotal role in the emergence of ego depletion. Depletion may emerge as a consequence of excessive focusing on ones regulatory efforts. When attention is directed away from the self-control action, ego depletion can be circumvented. Finally, indirect findings from Chapter 5 as well as research by Muraven and Slessareva (2003) suggest that motivational processes underlie the occurrence of ego depletion. No signs of ego depletion are present when people are reminded or confronted with regulatory goals. Based on these findings, we believe that the claim that energy is responsible for failure after repeated self-control is only part of the story. In addition, there is some potential danger that hides within the vague Freudian concept of energy. First of all, reasoning from a limited strength model may stimulate an inappropriate causal inference. That is, assuming that self-control consumes energy may lead to the conclusion that failure of self-control automatically reflects a loss of energy. However, it remains questionable whether decrement of self-control performance always reflects a decreased level of energy or an attempt to conserve energy. While some instances of self-control failure may indeed primarily occur as the result of a default depletion reaction, it seems likely that other instances indicate a conscious decision and are therefore rather determined by processes like justification and motivation. Secondly, the concept of energy does not contain any theoretical restrictions. As a consequence, it is possible to explain almost every finding, thereby restricting the insights provided by 
CHAPTER 7 | GENERAL DISCUSSION

future research. A possible way to overcome this restriction is simply by disconnecting the emergence of ego depletion from the strength model, rather than attempting to provide support for the model. In this way, a broader vision on self-control failure can be adopted that is more in line with human complexity and flexibility. 
SUMWARY 
Self-control, or self-regulation, refers to the extent to which an individual exerts control over his or her behaviour. Self-control has been defined as the override or inhibition of urges, behaviours or desires (Baumeister, Heatherton, \& Tice, 1994; Shallice \& Burgess, 1993) and can be regarded as a fundamental aspect of our daily life. On a daily basis we attempt to remain patient, suppress unwanted thoughts or resist temptations. Research has shown that being able to control oneself is a vital ability: good selfcontrol abilities are associated with a range of positive life circumstances. For instance, people who posses relatively good self-control abilities have fewer conflicts with family members, higher self-esteem, lower chances of developing psychopathological problems and a better emotional life (Tangney, Baumeister, \& Boone, 2004). In contrast, a lack of self-control has been found to be associated with various social and personal problems. Examples of such problems include obesity, unsafe sex, aggression and alcoholism (Baumeister, Heatherton, \& Tice, 1994). In sum, these findings suggest that self-control can be defined as a key factor for a successful life.

Despite the importance of self-control, we often fail to control ourselves successfully. An important question in this respect concerns the reason for this failure. According to the strength model (Muraven, Tice, \& Baumeister, 1998; Muraven \& Baumeister, 2000), self-control failure arises as a direct consequence of limited energy. In this view, self-control requires energy of which people have only a limited amount available. After an initial act of self-control, the energy resource becomes temporarily depleted leaving the individual in a state of ego depletion. Consequently, a subsequent self-control attempt results in impaired performance because of a lack of energy or strength. Today, a relatively large amount of studies have indeed provided support for the strength model. Examples of findings are that the regulation of thoughts (i.e., suppressing a specific thought), altering one's emotional state (i.e., suppressing happy or sad emotions), and decision making involve self-control and that each of these activities results in impaired performance on a second self-control activity (see for an overview, Schmeichel, \& Baumeister, 2004; Martijn, Alberts, \& De Vries, 2006). So far, most studies on ego depletion have focused on demonstrating that different behaviours and outcome measures are influenced by ego depletion. Identifying these different outcome measures can be considered as a valuable and necessary, however, it does not provide more insight in the processes that underlie self-control and ego depletion. At this point, little is known about the nature of limited strength and the psychological process underlying this limitation remains unclear. This dissertation addressed this issue by attempting to provide more insight in the processes that underlie self-con- 
trol performance and ego depletion. In doing so, we adopted a somewhat different perspective on self-control than the earlier mentioned strength model. Whereas the strength model mainly focuses on the limits of our self-control abilities, the current research was guided by a focus on the flexibility and relativity of regulatory capabilities. By focusing on possible ways to improve self-control and to circumvent ego depletion, it was argued that the strength model can be extended and room can be created for additional factors and processes that contribute to self-control performance.

The studies described in second chapter of this dissertation addressed the role of expectancies on self-control. Previous research findings suggest that self-control performance and the occurrence of ego depletion may be influenced by people's expectancies or schemata about self-control (Martijn, Tenbült, de Vries, Dreezens, \& Merckelbach, 2002; Mukhopadhyay \& Johar, 2005). The results of these studies imply that rather than the existence of a limited energy resource, the (naïve) expectancy that self-control is dependent on limited energy may explain self-control failure. After all, an individual who expects that self-control is a matter of limited energy is likely to be concerned with minimizing loss and the possible obstacles when exerting self-control. Such beliefs may cause one to quit self-control attempts sooner in order to minimize future failure. Holding and acting upon the belief that self-control is dependent on limited energy may therefore have serious implications for self-control performance and long term goal achievement. Three studies were conducted in order to gain more insight in the relation between self-control abilities and self-control related beliefs. More specifically, it was investigated whether there is a relation between the extent to which people believe that self-control is dependent on limited energy and their self-reported self-control abilities. To do so, a scale was developed to assess the extent to which participants believe that self-control is dependent on (limited) energy. In order to assess their self-control ability, participants completed the Self-control Scale (Tangney, Baumeister \& Boone, 2004) (Study 1 and Study 2) and the Barratt Impulsiveness Scale (Barratt, 1994) (Study 3). The results showed that stronger energy related beliefs of self-control are related to worse self-reported self-control abilities (Study 1 and Study 2) as well as higher levels of overall impulsiveness (Study 3). In sum, these findings imply that when considering self-control failure (and the emergence of ego depletion), it may be worthwhile to consider the beliefs that people hold about the operation of self-control. Changing the belief that self-control strongly depends on energy may help to improve regulatory performance and circumvent the occurrence of ego depletion. 
In Chapter 3, two studies were presented that addressed the issue of automaticity and self-control. More specifically, it was investigated whether the automatic emergence of ego depletion can be reduced or even eliminated by means of manipulations that are known to operate at the same automatic level as the occurrence of ego depletion itself. Whereas previous studies have demonstrated that self-control can be improved by means of explicit manipulations that require conscious attention or processing, the present experiments investigated whether the same effects can be accomplished by ways that do not require conscious attention or awareness. In two studies, the concept of persistence was unconsciously activated by means of different priming procedures. In Study 1, persistence was activated by means of a so-called Scrambled Sentence Task. Participants unscrambled 25 scrambled sentences of which 15 were related to persistence (e.g., "Peter keeps going" ); the other 10 were neutral and were not related to persistence or self-control (e.g., "Sam buys bread"). In Study 2, a visual priming procedure was used to unconsciously activate persistence. While participants were performing a physical self-control task, the experimenter activated a screensaver which appeared on a computer screen. In the persistence prime condition, the screensaver depicted a winning young man in a business suit and a logo saying: "www.you-cando-it.com, wallpapers and screensavers". In the neutral prime condition, the screensaver showed a picture of a vase and a similar logo saying: "www.myscreensaver.com, wallpapers and screensavers". In both studies it was found that depleted participants who were primed with persistence performed significantly better on a subsequent selfcontrol task than depleted participants who were exposed to a neutral prime. Priming of persistence caused initially depleted participants to overcome ego depletion: their performance on a subsequent self-control task remained equal. In contrast, depleted participants who were exposed to the neutral prime displayed the classic ego depletion pattern: their performance decreased significantly. According to the strength model, self-control entails a conscious, active process. These findings, however, illustrate that self-control may also be guided by processes that operate outside awareness.

Research presented in Chapter 4 investigated the role of attentional processes in self-control. More specifically, this chapter addressed the effect of focusing attention on the control demanding situation (internal focus or cognitive association) versus directing attention away from the self-control action (external focus). In the present experiment, participants were asked to perform two successive physical self-control tasks. An internal focus was induced by asking participants to pay attention to their arm and hand muscles that were involved while they were performing the second physical 
self-control task (internal focus or cognitive association). An external focus was induced by a distracting calculation task that was completed by participants during the exertion of the second physical self-control task. It was found that participants who were distracted by this calculation task (external focus) while exerting physical selfcontrol outperformed both participants who focused their attention on the physical self-control task (internal focus) as well as control participants who did not receive any focus instructions. However, no significant difference in self-control performance was found between participants in the internal focus condition and control participants. With regard to the strength model, these findings suggest that attentional processes play an important role in the emergence of ego depletion and the failure of self-control. The finding that both people who focused attention internally as well as control participants exhibited a decline in performance, might imply that the occurrence of ego depletion is partly mediated by ineffective or suboptimal attentional processes. Directing attention to the self-control task (and the regulatory efforts that are associated with this task), may be a default process or natural tendency that emerges when people exert self-control. When this default attentional process is replaced by a process that is not preliminary concerned with regulatory efforts, no negative influence on self-control performance seems to occur.

In Chapter 5, the influence of attentional processes on self-control performance was further elaborated. Previous studies investigating the effects of self-focused attention on self-control performance have revealed a mixed pattern of findings; part of these studies highlight the self-control enhancing effect of self-focused attention, while others illustrate the regulatory downside of self-directed attention. The study presented in Chapter 5 was designed to provide more insight in these contradictory findings. It was reasoned that it is critical to differentiate between distinct types of self-focused attention. On the one hand, self-focused attention may involve the type of attention that was addressed in the study described in Chapter 4, namely attention directed towards the self-control action and internal sensations during this action (cognitive association). On the other hand, self-focus may also involve what is known under the term self-awareness. When self-focus concerns self-awareness, people become aware of the self's existence in the world and the salience of an internalized, social and/or situational standard is increased. The study in Chapter 5 attempted to establish a direct comparison between both types of self-focused attention. In order to isolate the effect of cognitive association, both types of self-focus were combined. More specifically, some participants were exposed to a manipulation that increased only self- 
awareness, whereas others received a manipulation that increased both self-awareness and cognitive association. It was found that the type of self-focus determines the effect of self-focus on regulatory performance. Exposure to a self-awareness manipulation caused initially depleted participants to keep their physical self-control performances constant. This group outperformed both control participants as well as participants who were also exposed to the cognitive association manipulation by which attention was directed towards the self-control action. In line with the findings of Chapter 4, no improvement of self-control was observed when participants were highly self-aware but at the same time aware of internal states. In sum, the findings of Chapter 4 and 5 not only illustrate the importance of attentional processes in self-control and ego depletion but also demonstrate that the use of attentional strategies may help to overcome ego depletion.

In Chapter 6, self-control and ego depletion were presented in an applied context, namely criminality. It was argued that, in order to understand criminal behavior, one should not only focus on intentional crimes, which are characterized by organized or planned criminal activity, but should also consider the role of impulsive crimes. The latter involve a tendency to give in to violent or other disastrous impulses and strongly rely on momentary self-control. The results described in the present dissertation as well as other related findings were linked to criminality in order to gain more insight in the emergence of impulsive criminal behavior. Moreover, based on these findings, guidelines for preventing failure of impulse control and improvement of self-control performance were presented.

In Chapter 7, the main results of this thesis were summarized and discussed. The research presented in this dissertation has led to some new insights regarding the operation of self-control and ego depletion. Whereas the strength model regards self-control as a conscious, active process, the present findings illustrate that self-control may also be guided by processes that operate outside awareness. Unconscious activation of persistence can help people to overcome ego depletion and improve self-control performance. In addition, also attentional processes seem to play a key role in the emergence of ego depletion. The findings suggest that depletion emerges as a consequence of excessive focusing on ones regulatory efforts. When attention is directed away from the self-control action, ego depletion can be circumvented. Finally, the current studies on the role of expectancies suggest that beliefs on the operation of self-control may also underlie self-regulatory performance and ego depletion. Taken together, whereas the strength model stresses the limits of our capability to control ourselves, the present 
findings rather illustrate the flexibility and relativity of these limits and suggest that that self-control and the emergence of ego depletion is more dynamic than initially suggested. The current findings favor a broader vision on self-control failure that is more in line with human complexity and flexibility. 
SAMENVATING 
Zelfcontrole, ook wel zelfregulatie genoemd, verwijst naar de mate waarin een individu controle over zichzelf uitoefent. Zelfcontrole wordt gedefinieerd als het onderdrukken of inhiberen van impulsen, gedrag of verlangens (Baumeister, Heatherton, \& Tice, 1994; Shallice \& Burgess, 1993) en kan worden beschouwd als een fundamenteel aspect van ons dagelijkse leven. Vrijwel iedere dag ondernemen we pogingen om onszelf te beheersen, vervelende gedachten te onderdrukken en verleidingen te weerstaan. Ons vermogen tot zelfcontrole is een onmisbare eigenschap. Zo heeft recent onderzoek laten zien dat goede zelfcontrolevaardigheden samengaan met een keur van begerenswaardige levensomstandigheden: minder conflicten met vrienden en familie, hoger zelfvertrouwen, minder kans op psychopathologische problemen en een gezonder emotioneel leven (Tangney, Baumeister, \& Boone, 2004). Omgekeerd leidt een gebrek aan zelfcontrole tot allerlei sociale en persoonlijke problemen. Bekende voorbeelden van zulke problemen zijn overgewicht, onveilige seks, agressie en alcoholisme (Baumeister, Heatherton, \& Tice, 1994). Samenvattend zou men kunnen stellen dat zelfcontrole het sleutelwoord is voor een succesvol leven.

Ondanks het belang van zelfcontrole, slagen we er lang niet altijd in onszelf te controleren. Waarom vinden mensen het zo moeilijk zichzelf in de hand te houden? Een mogelijke verklaring voor het falen van zelfcontrole wordt gegeven door het zogenaamde gelimiteerde energie model. Diverse studies hebben aangetoond dat mensen die twee opeenvolgende controle taken verrichten, aanzienlijk slechter presteren op de tweede zelfcontroletaak. Volgens het zogenaamde gelimiteerde energiemodel ligt een centrale, beperkte energiebron ten grondslag aan deze bevinding (Baumeister, Muraven, \& Tice, 2000). Iedere zelfcontrolehandeling doet een beroep op deze beperkte energiebron, waardoor er steeds minder energie beschikbaar is voor volgende zelfcontroletaken. Deze tijdelijke uitputting van de beschikbare energie wordt aangeduid met de term ego depletion of wilsuitputting. In een toestand van ego depletion zullen mensen op volgende zelfcontroletaken minder goed presteren.

In de laatste jaren is er relatief veel onderzoek verricht naar het ego depletion fenomeen. Deze studies laten zien dat het principe van zelfcontrole als gelimiteerde energiebron in verschillende domeinen optreedt. Voorbeelden van bevindingen zijn dat de regulatie van gedachten (bijvoorbeeld het onderdrukken van een specifieke gedachte), het veranderen van een emotionele toestand (bijvoorbeeld het onderdrukken van positieve of negatieve emoties) en besluitvorming zelfcontrole vereisen en dat elk van deze activiteiten resulteert in verminderde prestatie op een tweede zelfcontrole handeling (voor een overzicht zie Schmeichel \& Baumeister, 2004; Martijn, Alberts, 
\& De Vries, 2006). De meeste studies die tot op heden zijn verricht naar het fenomeen ego depletion hebben voornamelijk gedemonstreerd dat verschillende gedragingen en uitkomstmaten worden beïnvloed door ego depletion. Hoewel de identificatie van deze verschillende gedragingen en uitkomstmaten als zinvol en waardevol kan worden, wordt er niet meer inzicht verkregen in de processen die ten grondslag liggen aan zelfcontrole en ego depletion. Er is dan ook relatief weinig bekend over de precieze aard van gelimiteerde energie en de psychologische processen die van invloed zijn op deze beperking. In het huidige proefschrift is er getracht meer inzicht te krijgen in de processen die ten grondslag liggen aan zelfcontrole en ego depletion. Om dit te bewerkstelligen is er een perspectief gehanteerd dat enigszins afwijkt van het eerder beschreven gelimiteerde energie model. Terwijl het gelimiteerde energie model zich voornamelijk richt op de grenzen van zelfcontrole, werd het huidige onderzoek gekenmerkt door een focus op de flexibiliteit en relativiteit van onze regulatieve capaciteit. De gedachtegang achter deze visie is dat door de aandacht te vestigen op mogelijke verbetering van zelfcontrole en vermijding van ego depletion, het gelimiteerde energie model kan worden uitgebreid en ruimte kan worden gecreëerd voor additionele factoren en processen die bijdragen aan zelfcontrole prestatie.

De studies die worden beschreven in het tweede hoofdstuk van dit proefschrift betreffen de rol van verwachtingen bij zelfcontrole. Eerdere bevindingen suggereren dat zelfcontrole prestatie en het optreden van ego depletion worden beïnvloed door de verwachtingen of schema's van mensen over zelfcontrole (Martijn, Tenbült, de Vries, Dreezens, \& Merckelbach, 2002; Mukhopadhyay \& Johar, 2005). De resultaten van deze studies suggereren dat wellicht niet in eerste instantie het bestaan van een gelimiteerde energiebron, maar eerder de verwachting dat zelfcontrole energie kost, ten grondslag ligt aan het falen van zelfcontrole. Immers, een individu dat sterke, op gelimiteerde energie gebaseerde verwachtingen hanteert, zal voornamelijk bij het uitvoeren van zelfcontrole meer gericht zijn op zuinigheid, minimalisatie van verlies en de mogelijke valkuilen bij het behalen van een doel. Dergelijke verwachtingen zullen aanzetten tot het eerder laten varen van zelfcontrole, om de kans op mogelijk toekomstig falen te minimaliseren. De verwachting dat zelfcontrole afhankelijk is van gelimiteerde energie kan daarom belangrijke implicaties hebben voor zelfcontroleprestatie en het bereiken van lange termijn doelen. Drie studies werden verricht om meer inzicht te verkrijgen in de relatie tussen verwachtingen over zelfcontrole en zelfcontrolevaardigheden. Er werd onderzocht of de mate waarin mensen verwachten dat zelfcontrole afhankelijk is van een gelimiteerde hoeveelheid energie, van 
invloed is op de inschatting van hun eigen zelfcontroleprestaties. Hiertoe werd een vragenlijst ontwikkeld om te meten in welke mate deelnemers verwachten dat zelfcontrole afhankelijk is van (gelimiteerde) energie. Om hun zelfcontrole vaardigheden in kaart te brengen dienden deelnemers de zogenaamde Self-control Scale (Tangney, Baumeister, \& Boone, 2004) (Studie 1 en Studie 2) en de Barratt Impulsiveness Scale (Barratt, 1994) (Studie 3) in te vullen. De resultaten toonden dat naarmate mensen meer geneigd zijn te denken over zelfcontrole in termen van (gelimiteerde) energie, zij relatief minder goede zelfcontrolevaardigheden (Studie 1 en Studie 2) en meer impulsiviteit (Studie 3) rapporteren. Samenvattend impliceren deze bevindingen dat wanneer men kijkt naar het falen van zelfcontrole (en het optreden van ego depletion), het waardevol zou kunnen zijn om de verwachtingen van mensen omtrent de werking van zelfcontrole in acht te nemen.

In hoofdstuk 3 werden twee studies gepresenteerd over automaticiteit en zelfcontrole. Er werd gekeken of het automatische optreden van ego depletion verminderd of zelfs geheel kan worden tegengegaan door gebruik te maken van manipulaties die op hetzelfde, automatische niveau opereren als het optreden van ego depletion zelf. Terwijl eerdere studies reeds hebben gedemonstreerd datzelfcontrolekan worden verbeterd middels expliciete manipulaties die bewuste aandacht of verwerking vereisen, is er in de huidige experimenten gekeken of dezelfde effecten kunnen worden bewerkstelligd op manieren die geen bewuste aandacht vereisen. In twee studies werd het concept doorzettingsvermogen onbewust geactiveerd door middel van verschillende priming procedures. In Studie 1 werd doorzettingsvermogen geactiveerd door gebruik te maken van een zogenaamde woordvolgordetaak (Scrambled Sentence Task). Deelnemers vormden 25 zinnen waarvan 15 zinnen gerelateerd waren aan doorzettingsvermogen (bijvoorbeeld "Peter gaat door"); de andere 10 zinnen waren neutraal van aard en niet gerelateerd aan doorzettingsvermogen of zelfcontrole (bijvoorbeeld "Sam koopt brood"). In Studie 2 werd een visuele priming procedure gebruikt om doorzettingsvermogen onbewust te activeren. Terwijl deelnemers een fysieke zelfcontrole taak uitvoerden activeerde de proefleider een screensaver die op een computer beeldscherm verscheen. De helft van de deelnemers kreeg een screensaver te zien met daarop een jonge man in pak die een hardloopwedstrijd wint met het logo "www.you-can-do-it.com, wallpapers and screensavers" (hoge toegankelijkheid van doorzettingsvermogen). De andere helft zag een screensaver van een neutrale afbeelding (een vaas) en een vergelijkbaar logo "www.myscreensaver. com, wallpapers and screensavers" (lage toegankelijkheid van doorzettingsvermo- 
gen). In beide studies werd gevonden dat uitgeputte deelnemers waarbij doorzettingsvermogen werd geactiveerd significant beter presteerden op een volgende zelfcontrole taak dan uitgeputte deelnemers die bloot werden gesteld aan een neutrale prime. Het activeren van doorzettingsvermogen leidde ertoe dat uitgeputte deelnemers ego depletion overwonnen: hun prestatie op een volgende zelfcontrole taak bleef gelijk. Uitgeputte deelnemers die werden blootgesteld aan de neutrale prime vertoonden daarentegen het klassieke ego depletion effect: hun prestatie daalde significant. Volgens het gelimiteerde energiemodel betreft zelfcontrole een bewust en actief proces. De huidige bevindingen illustreren echter dat zelfcontrole ook wordt gestuurd door processen die buiten ons bewustzijn opereren.

Onderzoek gepresenteerd in Hoofdstuk 4 betrof de rol van attentionele processen bij zelfcontrole. Er werd gekeken naar het effect van het richten van aandacht op de controle vereisende handeling (interne focus of cognitieve associatie) versus het afwenden van aandacht van de zelfcontrolehandeling (externe focus). In de huidige studie dienden proefpersonen achtereenvolgens twee fysieke zelfcontroletaken uit te voeren. Om een interne focus te induceren dienden proefpersonen zich tijdens het uitvoeren van de tweede fysieke zelfcontrole taak zo volledig mogelijk te concentreren op hun eigen lichaam en dan voornamelijk op de arm- en handspieren die gebruikt worden bij deze taak (interne focus of cognitieve associatie). Een externe focus werd geïnduceerd door een cognitief belastende rekentaak, die deelnemers dienden op te lossen tijdens het uitvoeren van de tweede fysieke zelfcontroletaak. De resultaten lieten zien dat deelnemers die tijdens het uitvoeren van zelfcontrole werden afgeleid door de rekentaak (externe focus) significant beter presteerden dan deelnemers die hun aandacht richtten op de fysieke zelfcontrole taak (interne focus) en deelnemers uit de controlegroep, die geen enkele focus instructie ontvingen. Echter, er werden geen significante verschillen in zelfcontrole prestatie gevonden voor deelnemers in de interne focus conditie en deelnemers in de controlegroep. Met betrekking tot het gelimiteerde energiemodel suggereren deze bevindingen dat aandachtsprocessen een belangrijke rol spelen bij de totstandkoming van ego depletion en het falen van zelfcontrole. De bevinding dat zowel deelnemers die hun aandacht intern vestigden als deelnemers uit de controlegroep een afname in prestatie vertoonden, zou kunnen betekenen dat het optreden van ego depletion gedeeltelijk wordt gemedieerd door ineffectieve of suboptimale aandachtsprocessen. Het richten van de aandacht op de zelfcontrolehandeling (en de moeite die hiermee gepaard gaat) zou een standaard proces of natuurlijke tendens kunnen zijn die optreedt wanneer mensen zelfcontrole uitvoeren. 
Wanneer dit standaard proces vervangen wordt door een proces dat niet in eerste instantie gericht is op de zelfcontrole handeling en de moeite die hiermee gepaard gaat blijkt er geen negatieve invloed op zelfcontrole prestatie plaats te vinden.

In Hoofdstuk 5 werd de invloed van aandachtsprocessen bij zelfcontrole nader onderzocht. Eerdere studies naar de rol van zelfgerichte aandacht bij zelfcontrole demonstreerden een niet-consistent patroon van bevindingen: sommigen van deze studies illustreren een zelfcontrole bevorderend effect van zelfgerichte aandacht, terwijl andere studies juist een negatieve invloed van zelfgerichte aandacht aantonen. Het hoofddoel van de studie die gepresenteerd werd in Hoofdstuk 5 was het verkrijgen van meer inzicht in deze tegengestelde bevindingen. Er werd verondersteld dat het essentieel is een onderscheid te maken tussen verschillende typen van zelfgerichte aandacht. Enerzijds kan zelfgerichte aandacht het type aandacht betreffen dat in Hoofdstuk 4 werd onderzocht, namelijk aandacht gericht op de zelfcontrolehandeling en de interne sensaties die aanwezig zijn tijdens deze handeling (cognitieve associatie). Anderzijds kan zelfgerichte aandacht ook betrekking hebben op zogenaamd zelfbewustzijn (self-awareness). Wanneer mensen in hoge mate zelfbewust zijn, worden zij bewust van hun bestaan in de wereld en worden bepaalde geïnternaliseerde sociale en/of situationele standaards in verhoogde mate saillant. De studie beschreven in Hoofdstuk 5 probeerde een directe vergelijking te maken tussen beide typen zelfgerichte aandacht. Om het effect van cognitieve associatie te isoleren, werden beide typen aandacht gecombineerd: sommige deelnemers werden blootgesteld aan een manipulatie die alleen zelfbewustzijn verhoogde, terwijl anderen een manipulatie ontvingen die zowel zelfbewustzijn als cognitieve associatie verhoogde. De bevindingen toonden aan dat het type zelfgerichte aandacht het effect van zelfgerichte aandacht op zelfcontrole prestatie bepaalt. Uitgeputte deelnemers die werden blootgesteld aan de zelfbewustzijn manipulatie wisten hun prestatie bij een volgende fysieke zelfcontrole taak constant te houden. Deze groep presteerde significant beter dan deelnemers uit de controlegroep en deelnemers die ook waren blootgesteld aan de cognitieve associatie manipulatie, waarbij de aandacht was gericht op de zelfcontrolehandeling. Overeenkomstig met de bevindingen van Hoofdstuk 4 werd er geen verbetering van zelfcontrole geconstateerd wanneer deelnemers in hoge mate zelfbewust waren en tevens zich bewust waren van interne sensaties (cognitieve associatie). Samenvattend tonen de bevindingen van Hoofdstuk 4 en 5 niet alleen het belang van aandachtsprocessen bij zelfcontrole, maar demonstreren zij ook dat het gebruik van aandachtsstrategieën kan helpen om ego depletion te overwinnen. 
In Hoofdstuk 6 werden zelfcontrole en ego depletion gepresenteerd in een toegepaste context, namelijk criminaliteit. Er werd verondersteld dat om crimineel gedrag te begrijpen, men zich niet moet beperken tot intentionele misdaden, die worden gekarakteriseerd door georganiseerde of geplande criminele activiteit, maar tevens moet richten op de rol van impulsieve misdaden. Impulsieve misdaden betreffen de neiging om toe te geven aan gewelddadige of andere ongewenste impulsen en zijn sterk afhankelijk van zelfcontrole. Om een inzicht te krijgen in de totstandkoming van crimineel gedrag werden zowel de bevindingen beschreven in het huidige proefschrift alsook andere gerelateerde bevindingen in verband gebracht met criminaliteit. Bovendien werden er, gebaseerd op deze bevindingen, mogelijke richtlijnen geboden om verlies van impulscontrole te voorkomen en verbetering van zelfcontrole prestatie te realiseren.

In Hoofdstuk 7 werden de belangrijkste bevindingen van dit proefschrift samengevat en bediscussieerd. Het onderzoek dat werd beschreven in het huidige proefschrift heeft tot enkele nieuwe inzichten geleid wat betreft zelfcontrole en het fenomeen ego depletion. Terwijl het gelimiteerde energiemodel zelfcontrole beschouwt als een bewust, actief proces, tonen de huidige bevindingen aan dat zelfcontrole wellicht ook wordt gestuurd door processen die opereren buiten ons bewustzijn. Onbewuste activatie van doorzettingsvermogen kan mensen helpen om ego depletion te overwinnen and zelfcontroleprestatie te verbeteren. Ook aandachtsprocessen blijken een belangrijke rol te spelen bij het optreden van ego depletion. De bevindingen suggereren dat ego depletion optreedt als gevolg van een excessieve focus op de zelfcontrole handeling en de moeite die hiermee gepaard gaat. Wanneer men aandacht van de zelfcontrole handeling afwendt, blijkt het optreden van ego depletion achterwege te blijven. Ten slotte impliceren de bevindingen omtrent de rol van verwachtingen dat ideeën over de werking van zelfcontrole van invloed zijn bij zelfcontroleprestatie en ego depletion.

Concluderend kan men stellen dat terwijl het gelimiteerde energie model de grenzen van ons vermogen tot zelfcontrole benadrukt, de huidige bevindingen eerder de flexibiliteit en relativiteit van deze grenzen benadrukken en bovendien suggereren dat het optreden van ego depletion dynamischer is dan men in eerste instantie veronderstelde. De bevindingen pleiten voor een bredere visie omtrent zelfcontrole die meer in overeenkomst is met de menselijke complexiteit en flexibiliteit. 
REFERENCES 
Ainslie, G. (1974). Impulse control in pigeons. Journal of the Experimental Analysis of Behavior, 21, 485489.

Ainslie, G., \& Hernstein, R. J. (1981). Preference reversal and delayed reinforcement. Animal Learning and Behavior, 9, 476-482.

Alberts, H., Martijn, C., \& de Vries, N. K. (2006). Differential effects of self-focused attention on self-control performance. Unpublished manuscript.

Alberts, H. J. E. M., Martijn, C., Nievelstein, F., Janssen, A., \& de Vries, N. K. (2006). Attentional focus and self-control perfomance. Manuscript submitted for publiction.

Alberts, H. J. E. M., Martijn, C., Greb, J., Merckelbach, H. \& de Vries, N. K. (in press). Carrying on or giving in: The role of automatic processes in overcoming ego depletion. British Journal of Social Psychology.

Akgun, S. (2004). The effects of situation and learned resourcefulness on coping responses. Social Behavior and Personality, 32, 441-448.

Araya, T., Akrami, N., Ekehammar, B., \& Hedlund, L. E. (2002). Reducing prejudice through priming of control-related words. Experimental Psychology, 49, 222-227.

Arneklev, B. J., Grasmick, H. G., Tittle, C. R., \& Bursik, R. J., Jr. (1993). Low self-control and imprudent behavior. Journal of Quantitative Criminology, 9, 225-247.

Aspinwall, L. G., \& Taylor, S. E. (1992). Modeling cognitive adaptation: A longitudinal investigation of the impact of individual differences and coping on college adjustment and performance. Journal of Personality and Social Psychology, 63, 989-1003.

Bailey, D. S., Leonard, K. E., Cranston, J. W., \& Taylor, S. P. (1983). Effects of alcohol and self-awareness on human physical aggression. Personality and Social Psychology Bulletin, 9, 289-295.

Baldwin, M. W., \& Holmes, J.G. (1987). Salient private audiences and awareness of the self. Journal of Personality and Social Psychology, 69, 254-268

Bandura, A. (1986). The explanatory and predictive scope of self-efficacy theory. Journal of Social and Clinical Psychology, 4, 359-373.

Bargh, J. A. (1990). Auto-motives: Preconscious determinants of social interaction. In E. T. Higgins \& R. M. Sorrentino (Eds.), Handbook of motivation and cognition: Vol. 2. Foundations of social behavior (pp. 93-130). New York: Guilford Press. 
Bargh, J. A., \& Chartrand, T. L. (2000). The mind in the middle: A practical guide to priming and automaticity research. In H. T. Reis \& C. M. Judd (Eds.), Handbook of research methods in social and personality psychology (pp. 253-285). New York: Cambridge University Press.

Bargh, J. A., Chen, M., \& Burrows, L. (1996). Automaticity of social behavior: Direct effects of trait construct and stereotype activation on action. Journal of Personality and Social Psychology, 71, 230-244.

Bargh, J. A., Gollwitzer, P. M., Lee-Chai, A., Barndollar, K., \& Trotschel, R. (2001). The automated will: Nonconscious activation and pursuit of behavioral goals. Journal of Personality and Social Psychology, 81, 1014-1027.

Barkley, R. A. (1997). ADHD and the nature of self-control. New York: Guilford Press.

Barratt, E. S. (1994). Impulsiveness and aggression. In J. Monahan \& H. J. Steadman (Eds.), Violence and mental disorder: Developments in risk assessment (pp. 6179). Chicago: University of Chicago Press.

Baumeister, R. F. (2002). Yielding to temptation: Self-control failure, impulsive purchasing, and consumer behavior. Journal of Consumer Research, 28, 670-676.

Baumeister, R. F., Bratslavsky, E., Muraven, M., \& Tice, D. M. (1998). Ego depletion: Is the active self a limited resource? Journal of Personality and Social Psychology, 74, 1252-1265.

Baumeister, R. F., \& Exline, J. J. (2000). Self-control, morality, and human strength. Journal of Social and Clinical Psychology, 19, 29-42.

Baumeister, R. F., Heatherton, T. F., \& Tice, D. M. (1994). Losing control: How and why people fail at self-regulation. San Diego, CA: Academic Press.

Baumeister, R. F., Muraven, M., \& Tice, D. M. (2000). Ego depletion: A resource model of volition, self-regulation, and controlled processing. Social Cognition, 18, 130 150.

Baumeister, R. F., \& Showers, C. J. (1986). A review of paradoxical performance effects: Choking under pressure in sports and mental tests. European Journal of Social Psychology, 16, 361-383.

Bechara, A., Damasio, A. R., Damasio, H., \& Anderson, S. W. (1994). Insensitivity to future consequences following damage to human prefrontal cortex. Cognition, 50, 7-15.

Berkowitz, L. (1978). Is criminal violence normative behavior? Hostile and instrumental aggression in violent incidents. Journal of Research in Crime and Delinquency, 15, 148-161. 
Boekaerts, M., Pintrich, P. R., \& Zeidner, M. (2000). Handbook of self-regulation. San Diego, CA: Academic Press.

Bouffard, T., Bouchard, M., Goulet, G., Denoncourt, I., \& Couture, N. (2005) Influence of achievement goals and self-efficacy on students' self-regulation and performance. International Journal of Psychology, 40, 373-384.

Brewer, M. B., \& Gardner, W. (1996). Who is this "We"? Levels of collective identity and self representations. Journal of Personality and Social Psychology, 71, 83-93.

Brunstein, J. C., \& Olbrich, E. (1985). Personal helplessness and action control:Analysis of achievement-related cognitions, self-assessments, and performance. Journal of Personality and Social Psychology, 48, 1540-1551.

Burton, V. S., Cullen, F. T., Evans, T. D., Alarid, L. F., \& Dunaway, R. G. (1998).Gender, self-control, and crime. Journal of Research in Crime and Delinquency, 35, 123147.

Bushman, B. J., Baumeister, R. F., \& Phillips, C. M. (2001). Do people aggress to improve their mood? Catharsis beliefs, affect regulation opportunity, and aggressive responding. Journal of Personality and Social Psychology, 81, 17-32.

Buss, A. H. (1980). Self-consciousness and social anxiety. San Francisco: Freeman.

Carver, C. S. (2002). Self-awareness. In M. R. Leary \& J. Tangney (Eds.), Handbook of self and identity (pp. 179-196). New York: Guilford Press.

Carver, C. S., \& Scheier, M. S. (1981). Attention and self-regulation: A control theory approach to human behavior. New York: Springer-Verlag.

Carver, C. S., \& Scheier, M. E (1990). Principles of self-regulation. In E.T. Higgins \& R. M. Sorrentino (Eds.), Handbook of motivation and cognition (pp. 3-52). New York: Guilford Press.

Cervone, D. (1996). People who fail at self-regulation: What should we think of them and how? Psychological Inquiry, 7, 40-46.

Chaiken, S., \& Trope, Y. (1999). Dual-process models in social psychology. New York: Guilford.

Ciarocco, N. J., Sommer, K. L., \& Baumeister, R. F. (2001). Ostracism and ego depletion: The strains of silence. Personality and Social Psychology Bulletin, 27, 1156-1163.

Cochran, J. K., Wood, P. B., \& Sellers, C. S. (1998). Academic dishonesty and low selfcontrol: An empirical test of a general theory of crime. Deviant Behavior, 19, 227255. 
De Jong, P. F., \& Das-Smaal, E. A. (1990). The Star Counting Test: An attention test for children. Personality and Individual Differences, 11, 597-604.

Dewsbury, D. A. (1981). Effects of novelty on copulatory behavior: The Coolidge effect and related phenomena. Psychological Bulletin, 89, 464-482.

Duval, S., \& Wicklund, R. (1972). A theory of objective self awareness. Oxford: Academic Press.

Dweck, C. S., Chui, C., \& Hong, C. (1995). Implicit theories and their role in judgments and reactions: A world from two perspectives. Psychological Inquiry, 6, 267-285.

Elster, J. (1979), Ulysses and the sirens: Studies in rationality and irrationality. New York: Cambridge University Press.

Faber, R. J., \& Vohs, K. D. (2004). To buy or not to buy? Self-control and self-regulatory failure in purchase behavior. In K. D. Vohs \& R. F. Baumeister (Eds.), Handbook of self-regulation (pp. 509-524). New York: Guilford Press.

Fazio, R. H. (1990). Multiple processes by which attitudes guide behavior: The MODE model as an integrative framework. In M. P. Zanna (Ed.), Advances in experimental social psychology (Vol. 23, pp. 75-109). New York: Academic Press.

Finkel, E. J., \& Campbell, W. K. (2001). Self-control and accommodation in close relationships: An interdependence analysis. Journal of Personality and Social Psychology, 81, 263-277.

Forde, D. R., \& Kennedy, L. W. (1997). Risky lifestyles, routine activities, and the general theory of crime. Justice Quarterly, 14, 265-294.

Furnham, A. F. (1988). Lay theories: Everyday understanding of problems in the social sciences. Oxford: Pergamon Press.

Gibbs, J. J., \& Giever, D. (1995). Self-control and its manifestations among university students: An empirical test of Gottfredson and Hirschi's general theory. Justice Quarterly, 12, 231- 255.

Gibbs, J. J., Giever, D., \& Martin, J. S. (1998). Parental management and self-control: An empirical test of Gottfredson and Hirschi's general theory. Journal of Research in Crime and Delinquency, 35, 40-70.

Gill, D. L., \& Strom, E. H. (1985). The effect of attentional focus on performance of an endurance task. International Journal of Sport Psychology, 16, 217-223.

Goode, K. T., \& Roth, D. L. (1993). Factor analysis of cognitions during running: Association with mood change. Journal of Sport and Exercise Psychology, 15, 375389. 
Gollwitzer, P. M. (1999). Implementation intentions: Strong effects of simple plans. American Psychologist, 54, 493-503.

Gollwitzer, P. M., \& Brandstätter, V. (1997). Implementation intentions and effective goal pursuit. Journal of Personality and Social Psychology, 73, 186199.

Gottfredson, M., \& Hirschi, T. (1990). A General Theory of Crime. Palo Alto, California: Stanford University Press.

Grasmick, H. G., Tittle, C. R., Bursik, R. J., \& Arneklev, B. K. (1993). Testing the core implications of Gottfredson and Hirschi's general theory of crime. Journal of Research in Crime and Delinquency, 30, 5-29.

Green, L., \& Myerson, J., (1993). Alternative frameworks for the analysis of self-control. Behavior and philosophy, 21, 37-47.

Hay, C. (2001). Parenting, self-control, and delinquency: A test of self-control theory. Criminology, 39, 707-736.

Heatherton, T. F., Polivy, J., Herman, C. P, Baumeister, R. F. (1993). Self-awareness, task failure, and disinhibition: How attentional focus affects eating. Journal of Personality, 61, 49-61.

Hevey, D., Smith, M., \& McGee, H. M. (1998). Self-efficacy and health behaviour: A review. Irish Journal of Psychology, 19, 248-273.

Higgins, G. E., \& Makin, D. A. (2004). Self-control, deviant peers, and software piracy. Psychological Reports, 95, 921-931.

Hofmann, W., Rauch, W., \& Gawronski, B. (in press). And deplete us not into temptation: automatic attitudes, dietary restraint, and self-regulatory resources as determinants of eating behavior. Journal of Experimental Social Psychology.

Holding, D. H. (1983). Fatigue. In G. R. J. Hockey (Ed.), Stress and fatigue in human performance (pp. 145-167). Chichester: John Wiley.

Hong, E. (1995). A structural comparison between state and trait self-regulation models. Applied Cognitive Psychology, 9, 333-349.

Hong, E. (1998). Differential stability of state and trait self-regulation in academic performance. Journal of Educational Research, 91, 148-158.

Hong, Y. Y., Chiu, Y. Y., \& Dweck, C. S. (1995). Implicit theories of intelligence: Reconsidering the role of confidence in achievement motivation. In M. Kernis (Ed.), Efficacy, agency, and self-esteem (pp. 197-216). New York: Plenum.

Hull, J. G. (1981). A self-awareness model of the causes and effects of alcohol consumption. Journal of Abnormal Psychology, 90, 586-600. 
Hutton, D. G., \& Baumeister, R. F. (1992). Self-awareness and attitude change: Seeing oneself on the central route to persuasion. Personality and Social Psychology Bulletin, 18, 68-75.

Jackson, R. C., Ashford, K. J., \& Norsworthy, G. (2006). Attentional Focus, dispositional reinvestment and skilled motor performance under pressure. Journal of Sport and Exercise Psychology, 28, 49-68.

Junger, M., West, R., \& Timman, R. (2001). Crime and risky behavior in traffic: An example of cross-situational consistency. Journal of Research in Crime and Delinquency, 38, 439-459.

Karniol, R., \& Miller, D. T. (1983). Why not wait? A cognitive model of self-imposed delay termination. Journal of Personality and Social Psychology, 45, 935-942.

Katz, J. (1988). Seductions of crime: The moral and sensual attractions of doing evil. New York: Basic Books.

Kennett, D. J., Ackerman, M. (1995). Importance of learned resourcefulness to weight loss and early success during maintenance: Preliminary evidence. Patient Education and Counseling, 25, 197-203.

Kirsch, I., Mearns, J., \& Catanzaro, S. J. (1990). Mood regulation expectancies as determinants of depression in college students. Journal of Counseling Psychology, 37, 306-312.

Kitsantas, A. (2000).The role of self-regulation strategies and self-efficacy perceptions in successful weight loss maintenance. Psychology and Health, 15, 811-820.

Kuhl, J. (1982). Action vs. state-orientation as a mediator between motivation and action. In W. Hacker, W. Volpert, \& M. Von Cranach (Eds.), Cognitive and motivational aspects of action (pp. 67-85). Berlin: VEB.

Kuhl, J. (1984). Volitional aspects of achievement motivation and learned helplessness: Toward a comprehensive theory of action control. In: B. A. Maher (Ed.), Progress in experimental personality research (pp. 99-171). New York: Academic Press.

Larson, R. (1989). Is feeling 'in control' related to happiness in daily life? Psychological Reports, 64, 775-784.

Larson, R. (1995). Secrets in the bedroom: Adolescents' private use of media. Journal of Youth and Adolescence, 24, 535-550.

Lu, L., \& Shih, J.B. (1997). Sources of Happiness: A Qualitative Approach. Journal of Social Psychology, 137, 181-187. 
LaGrange, T. C. \& Silverma, R. A. (1999). Low self-control and opportunity: Testing the general theory of crime as an explanation for gender differences in delinquency. Criminology, 37, 41-72.

Langer, E. J. (1989). Mindfulness. Reading, MA: Addison-Wesley.

Lent, R. W., Brown, S. D., \& Larkin, K. C. (1984). Relation of self-efficacy expectations to academic achievement and persistence. Journal of Counseling Psychology, 31, 356-362.

Lent, R. W., \& Hackett, G. (1987). Career self-efficacy: Empirical status and future directions. Journal of Vocational Behavior, 30, 347-382.

Liebert, R. M., and Spiegler, M. D. (1994). Personality: Strategies and issues. California: Brooks/Cole Publishing.

Logue, A. W. (1988). Research on self-control: an integrating framework. Behavioral and Brain Sciences, 11, 665-709.

Longshore, D., Stein, J. A., \& Turner, S. (1998). Reliability and validity of a self-control measure: A rejoinder. Criminology, 36, 175-182.

Macrae, C. N., Bodenhausen, G. V., \& Milne, A. B. (1998). Saying no to unwanted thoughts: Self-focus and the regulation of mental life. Journal of Personality and Social Psychology, 74, 578-589.

Mayer, J. D., \& Gaschke, Y. N. (1988). The experience and meta-experience of mood. Journal of Personality and Social Psychology, 55, 102-111.

Martijn, C., Alberts, H., \& de Vries, N. K. (2006). Maintaining self-control: The role of expectancies. In D. de Ridder \& J. de Wit (Eds.). Self-regulation in health behavior. Chichester UK: Wiley.

Martijn, C., Alberts, H., \& Goedegebuure, F. (2006). Positive self-control feedback eliminates ego depletion. Unpublished Manuscript.

Martijn, C., Alberts, H., Sheeran, P., Peters, G. Y., Mikolajczak, J. \& de Vries (2007). Frustrated actions, persistent minds: Implementation intentions affect tenacious goal striving. Manuscript submitted for publication.

Martijn, C., Huijts, A., Alberts, H., Merckelbach, H., \& de Vries, N. K. (in press). Keeping on or giving in: Accessibility of "perseverance" and its effect on successive selfcontrol tasks. European Journal of Social Psychology.

Martijn, C., Tenbült, P., Merckelbach, H., Dreezens, E., \& de Vries, N.K. (2002). Getting a grip on ourselves: Challenging expectancies about loss of energy after selfcontrol. Social Cognition, 20, 441-458. 
Masters, K. S., \& Ogles, B. M. (1998). Associative and dissociative cognitive strategies in exercise and running: 20 years later, what do we know? Sport Psychologist, 12, 253-270.

Mayer, J. D., \& Gaschke, Y. N. (1988). The experience and meta-experience of mood. Journal of Personality and Social Psychology, 55, 102-111.

Mearns, J. (1991). Coping with a breakup: Negative mood regulation expectancies and depression following the end of a romantic relationship. Journal of Personality and Social Psychology, 60, 327-334.

Mercer, J., McNevin, N., Guadagnoli, M. A. (2004). Reciprocal influences of attentional focus on postural and suprapostural task performance. Journal of Motor Behavior, 36, 189-199.

Mischel, W. (1974). Processes in delay of gratification. In L. Berkowitz (Ed.), Advances in Experimental Social Psychology (pp. 249-292). San Diego, CA: Academic Press.

Mischel, W., Ebbesen, E. B. (1970). Attention in delay of gratification. Journal of Personality and Social Psychology, 16, 329-337.

Mischel, W., Ebbesen, E. W., \& Zeiss, A. R. (1972). Cognitive and attentional mechanisms in delay of gratification. Journal of Personality and Social Psychology, 21, 204-218.

Mischel, W., Shoda, Y., \& Peake, P. K. (1988). The nature of adolescent competencies predicted by preschool delay of gratification. Journal of Personality and Social Psychology, 54, 687-696.

Monteith, M. J., Devine, P. G., \& Zuwerink, J. R. (1993). Self-directed versus otherdirected affect as a consequence of prejudice-related discrepancies. Journal of Personality and Social Psychology, 64, 198-210.

Mukhopadhyay , A., \& Johar, G. V. (2005). Where there is a will, is there a way? Effects of lay theories of self-control on setting and keeping resolutions. Journal of Consumer Research, 31, 779-786.

Mullen, B. (1986). Atrocity as a function of lynch mob composition: A self attention perspective. Journal of Personality and Social Psychology, 12, 187-197.

Muraven, M., \& Baumeister, R. F. (2000). Self-regulation and depletion of limited resources: Does self-control resemble a muscle? Psychological Bulletin, 126, 247259.

Muraven, M., Baumeister, R., \& Tice, D. M. (1999). Longitudinal improvement of self-regulation through practice: Building self-control strength through repeated exercise. Journal of Social Psychology, 139, 446-457. 
Muraven, M., Collins, R. L., \& Nienhaus, K. (2002). Self-control and alcohol restraint: an initial application of the self-control strength model. Psychology of addictive behaviors. Journal of the Society of Psychologists in Addictive Behaviors, 16, 113120.

Muraven, M., \& Slessareva, E. (2003). Mechanism of self-control failure: Motivation and limited resources. Personality and Social Psychology Bulletin, 29, 894-906.

Muraven, M., Tice, D. M., \& Baumeister, R. F. (1998). Self-control as limited resource: Regulatory depletion patterns. Journal of Experimental and Social Psychology, 74, 774-789.

Nagin, D. S., \& Paternoster, R. (1993). Enduring individual differences and rational choice theories of crime. Law and Society Review, 27, 467- 496.

Nagin, S., \& Pogarsky, G. (2004). Time and punishment: Delayed consequences and criminal behavior. Journal of Quantitative Criminology, 20, 295-317.

Nesselroade, J. R., \& Bartsch, T. W. (1977). Multivariate perspectives on the construct validity of the trait-state distinction. In R. B. Cattell \& R. M. Dreger (Eds.), Handbook of modern personality theory (pp. 221-238). Washington, DC: Hemisphere.

Nolen-Hoeksema, S. (1998). Ruminative coping with depression. In J. Heckhausen \& C. S. Dweck (Eds.), Motivation and self-regulation across the life span (pp. 237256). New York: Cambridge University Press.

Oaten, M., \& Cheng, K. (2005). Academic examination stress impairs self-control. Journal of Social and Clinical Psychology, 24, 254-279.

Oaten, M., \& Cheng, K. (2006). Improved self-control: The benefits of a regular program of academic study. Basic and Applied Social Psychology, 28, 1-16.

Oettingen, G., Pak, H., \& Schnetter, K. (2001). Self-regulation of goal-setting: Turning free fantasies about the future into binding goals. Journal of Personality and Social Psychology, 80, 736-753.

Oyserman, D., Terry, K., \& Bybee, D. (2002). A possible selves intervention to enhance school involvement. Journal of Adolescence, 24, 313-326.

Papies, E. K., Stroebe, W., \& Aarts, H. (2006). The allure of forbidden food: on the role of attention in self-regulation. Manuscript submitted for publication.

Piquero, A. R., \& Tibbetts, S. (1996). Specifying the direct and indirect effects of low self-control and situational factors in decision-making: Toward a more complete model of rational offending. Justice Quarterly, 13, 481- 510. 
Polakowski, M. (1994). Linking self- and social control with deviance: Illuminating the structure underlying a general theory of crime and its relation to deviant activity. Journal of Quantitative Criminology, 10, 41- 78.

Rodriguez, M. L., Mischel, W., \& Shoda, Y. (1989). Cognitive person variables in the delay of gratification of older children at risk. Journal of Personality and Social Psychology, 57, 358-367.

Rosenbaum, M. (1990). The role of learned resourcefulness in self-control of health behavior. In M. Rosenbaum (Ed.), Learned resourcefulness: On coping skills, self-control and adaptive behavior (pp. 4-25). New York: SpringerVerlag.

Rosenbaum, M. (2000). The self-regulation of experience: openness and construction. In: P. Dewe, A. M. Leiter \& T. Cox (Eds.), Coping and health in organizations (pp. 51-67). London: Taylor \& Francis.

Rosenbaum, M., \& Ben-Ari, K. (1985). Learned helplessness and learned resourcefulness: Effects of noncontingent success and failure on individuals differing in self-control skills. Journal of Personality and Social Psychology, 48, 198-215.

Santrock, J. (1976). Affect and facilitative self-control: Influence of ecological setting, cognition, and social agent. Journal of Educational Psychology, 68, 529-535.

Schmeichel, B. J., Vohs, K. D., \& Baumeister, R. F. (2003). Intellectual performance and ego depletion: Role of the self in logical reasoning and other information processing. Journal of Personality and Social Psychology, 85, 33-46.

Scott, Jr., R. F., Hawkins, Jr., R. D., \& Farnsworth, M. (1994). Operation kick-it: Texas prisoners rehabilitate themselves by dissuading others. Journal of Offender Rehabilitation, 20, 207-215.

Sellers, C. S. (1999). Self-control and intimate violence: An examination of the scope and specification of the general theory of crime. Criminology, 37, 375-404.

Shah, J. Y., Friedman, R., \& Kruglanski, A. W. (2002). Forgetting all else: On the antecedents and consequences of goal shielding. Journal of Personality and Social Psychology, 83, 1261-1280.

Shah, J. Y., \& Kruglanski, A. W. (2002). Priming against your will: How goal pursuit is affected by accessible alternatives. Journal of Experimental Social Psychology, $38,368-383$. 
Shallice, T., \& Burgess, P. (1993). Supervisory control of action and thought selection. In P. Baddeley \& L. Weiskrantz (Eds.), Attention: Selection, awareness, and control (pp. 171-187). Oxford: Oxford University Press.

Shoda, Y., Mischel, W., \& Peake, P. K. (1990). Predicting adolescent cognitive and social competence from preschool delay of gratification: Identifying diagnostic conditions. Developmental Psychology, 26, 978-986.

Srull, T. K., \& Wyer, R. S., Jr (1979). The role of category accessibility in the interpretation of information about persons: Some determinants and implication. Journal of Personality and Social Psychology, 37, 1660-1667.

Stark, R. (1989). Sociology. Belmont: Wadsworth.

Steele, C. M., \& Southwick, L. (1985). Alcohol and social behavior I: The mediating role of inhibitory conflict. Journal of Personality and Social Psychology, 48, 1834.

Strack, F., \& Deutsch, R. (2004). Reflective and impulsive determinants of social behavior. Personality and Social Psychology Review, 8, 220-247.

Stretcher, V. J., DeVillis, B. M., Becker, M. H., \& Rosenstock, I. M. (1986). The role of selfefficacy in achieving health behavior change. Health Education Quarterly, 13, 7391.

Stucke, T. S. \& Baumeister, R. F. (2006). Ego depletion and aggressive behavior: Is the inhibition of agression a limited resource? European Journal of Social Psychology, 36, 1-13.

Tangney, J. P., Baumeister, R. F., Boone, A. L. (2004). High self-control predicts good adjustment, less pathology, better grades, and interpersonal success. Journal of Personality, 72, 271-322.

Tice, D. M., Bratslavsky, E., \& Baumeister, R. F. (2001). Emotional distress regulation takes precedence over impulse control: If you feel bad, do it! Journal of Personality and Social Psychology, 80, 53-67.

Utz, S. (2004). Self-activation is a two-edged sword: The effects of I primes on cooperation. Journal of Experimental Social Psychology, 40, 769-776.

Vazsonyi, A. T., Pickering, L. E., Junger, M., \& Hessing, D. (2001). An empirical test of a general theory of crime: A four-nation comparative study of self-control and the prediction of deviance. Journal of Research in Crime and Delinquency, 38, 91131. 
Verplanken, B., \& Holland, R. W. (2002). Motivated decision making: Effects of activation and self-centrality of values on choices and behavior. Journal of Personality and Social Psychology, 82, 434-447.

Vohs, K. D. \& Baumeister, R. F. (2004). Depletion of self-regulatory resources makes people selfish. Unpublished manuscript, University of British Columbia, Vancouver, BC, Canada.

Vohs, K. D., \& Schmeichel, B. J. (2003). Self-regulation and extended now: Controlling the self alters the subjective experience of time. Journal of Personality and Social Psychology, 85, 217-230.

Webb, T. L., \& Sheeran, P. (2003). Can implementation intentions help to overcome ego depletion? Journal of Experimental Social Psychology, 39, 279-286.

Wegner, D. M. (1994). Ironic processes of mental control. Psychological Review, 16, 34-52.

Wegner, D. M., Ansfield, M., \& Pilloff, D. (1998). The putt and the pendulum: Ironic effects of mental control action. Psychological Science, 9, 196-199.

Wegner, D. M., Broome, A., \& Blumberg, S. J. (1997). Ironic effects of trying to relax under stress. Behavior Research and Therapy, 35, 11-21.

Wegner, D. M., Erber, R., \& Zanakos, S. (1993). Ironic processes in the mental control of mood and mood-related thought. Journal of Personality and Social Psychology, 65, 1093-1104.

Wegner, D. M., Schneider, D. J., Carter, S., \& White, L. (1987). Paradoxical effects of thought suppression. Journal of Personality and Social Psychology, 53, 5-13.

Wood, P. B., Pfefferbaum, B., \& Arneklev, B. J. (1993). Risk-taking and self-control: Social psychological correlates of delinquency. Journal of Crime and Justice, 16, 111- 130.

Wrisberg, C. A., Franks, B. D., Birdwell, M. W. \& High, D. M. (1988). Physiological and psychological responses to exercise with an induced attentional focus. Perceptual and Motor Skills, 66, 603-616.

Yates, J. F., \& Revelle, G. L. (1979). Processes operative during delay of gratification. Motivation and Emotion, 3, 103-115. 
DANKWOORD 
Graag wil ik een aantal mensen bedanken voor hun onmisbare rol bij de totstandkoming van dit proefschrift:

Carolien, je was voor mij een geweldige begeleider. Naast jouw deskundigheid stond je altijd voor me klaar, hielp je tegenslagen te relativeren door je gevoel voor humor en vervulde je de taak van beschermer wanneer nevenwerkzaamheden de overhand dreigden te krijgen. Dank voor alles!

Nanne en Harald; dank voor jullie visie en expertise die onmisbaar waren bij de cruciale momenten tijdens het project!

Mijn collega's: jullie waren zonder twijfel een van de belangrijkste redenen voor het feit dat ik dagelijks met plezier kwam werken. Dank hiervoor!

Saskia, dank voor het nakijken van het proefschrift en natuurlijk voor het feit dat je zo'n geweldige kamergenoot bent!

Laurens, dank voor je hulp bij de moeilijkere vraagstukken van mijn onderzoek. Je gestructureerde wiskundige blik wist orde te scheppen in ogenschijnlijke onoplosbaarheden.

Mijn vrienden: jullie herinnerden mij er aan wat echt belangrijk is in het leven.

Mijn ouders: buiten het feit dat jullie mij alle mogelijkheden hebben gegeven om te kunnen studeren, waren jullie steun en toeverlaat, dank!

Dorientje, je was en bent voor mij de "backbone". Er onvoorwaardelijk voor iemand zijn is iets waar geen dank groot genoeg voor is. 


\section{CURRICULUM VITAE}


Hugo Alberts was born on March 29, 1980, in Heerlen, the Netherlands. In July 1998, he graduated from secondary school (VWO, Sophianum College in Gulpen). In September 1998, he started studying psychology at Maastricht University. He graduated in August 2002 and received his master's degree in cognitive psychology. In December 2002, he started his PhD project at the Faculty of Psychology at Maastricht University. Since March 2007 he is a postdoctoral fellow at the Faculty of Psychology in Maastricht.

\section{Publications}

Alberts, H., \& de Cremer, D. (2002). Positieve emotionele reacties en procedurele rechtvaardigheid: De effecten van inspraak en selectie van leiderschap als functie van de behoefte ergens bij te horen. In E. Kluwer, E. van Dijk \& D. Wigboldus (Eds.), Jaarboek Sociale Psychologie. Delft: Eburon.

Alberts, H., Greb, J. \& Martijn, C. (2004). "You can do it.com!": Is zelfcontrole een kwestie van de juiste activatie? In E. Kluwer, E. van Dijk \& D. Wigboldus (Eds.), Jaarboek Sociale Psychologie. Delft: Eburon.

Alberts, H., Maroofi, S. \& Martijn, C. (2006). De relatie tussen verwachtingen en interpersoonlijke verschillen in zelfcontrole. In E. Kluwer, E. van Dijk \& D. Wigboldus (Eds.), Jaarboek Sociale Psychologie. Delft: Eburon.

Alberts, H. J. E. M., Martijn, C., \& de Vries, N. (2006). Voorbij de grenzen van beperkte energie. De Psycholoog, 41, 588-593.

Alberts, H. J. E. M., Martijn, C., Greb, J., Merckelbach, H. \& de Vries, N.K. (in press). Carrying on or giving in: The role of automatic processes in overcoming ego depletion. British Journal of Social Psychology.

Alberts, H. J. E. M., Panahi, R. M. S. \& Martijn, C. (2006). Understanding impulsive crime: The role of state self-control. Verlag für Polizeiwissenschaft, 3, 3043.

De Cremer, D., \& Alberts, H. J. E. M. (2002). When procedural fairness does not influence how positive I feel: The effects of voice and leader selection as a function of belongingness need. European Journal of Social Psychology, 34, 333-344.

Guerrieri, R., Nederkoorn, C., Stankiewicz, K., Alberts, H., Geschwind, N., Martijn, C., et al. (in press). The influence of trait and induced state impulsivity on food intake in normal-weight healthy women. Appetite. 
Martijn, C., Alberts, H., \& de Vries, N. K. (2006). Maintaining self-control: The role of expectancies. In: D. de Ridder \& J. de Wit (Eds.). Self-regulation in health behavior. Chichester UK: Wiley.

Martijn, C., Alberts, H. J. E. M., Peters, G. J., Mikolajczak, J., de Vries, N. K. \& Sheeran, P. (in press). Over doelen, frustraties en doorzetten: Leiden implementatie intenties tot volhardend doelgericht gedrag? In C. Van Laar, R. Ruijter, J. Karremans, W. Van Rijswijk, \& F. Van Harreveld (Eds.), Jaarboek sociale psychologie. Groningen, The Netherlands: ASPO Pers.

Martijn, C., Huijts, A., Alberts, H., Merckelbach, H., \& deVries, N. K. (in press). Keeping on or giving in: Accessibility of "perseverance" and its effect on successive selfcontrol tasks. European Journal of Social Psychology.

Nievelstein, F., Alberts, H., Jansen, A., van Doorn, R., \& Martijn, C. (2004). Het gaat beter als je aan iets anders denkt: De rol van externe versus zelffocus in zelf controleprocessen. In E. Kluwer, E. van Dijk \& D. Wigboldus (Eds.), Jaarboek Sociale Psychologie. Delft: Eburon.

\section{Submitted manuscripts}

Alberts, H. J. E. M., Martijn, C., \& de Vries, N.K. (2007). Differential effects of self-focused attention on self-regulatory performance. Manuscript submitted for publication.

Alberts, H. J. E. M., Martijn, C., \& De Vries, N.K. (2007). Self-control related expectancies and self-control abilities.Manuscript submitted for publication.

Alberts, H. J. E. M., Martijn, C., Nievelstein, F., Jansen, A., \& de Vries, N.K. (2006). Attentional focus and self-control performance. Manuscript submitted for publication.

Martijn,C.,H.J.E.M.Alberts, Sheeran, S.,Peters, G.J.Y., \&deVries, N.K. (2006).Frustrated actions, persistent minds: Implementation intentions affect tenacious goal-striving. Manuscript submitted for publication.

\section{Conference presentations}

Alberts, H., Martijn, C., \& de Vries, N. K. (2006). Self-controlandinterpersonaldifferences: The role of (implicit) cognition in self-control performances. Paper presented at the 8th conference of the European Social Cognition Network (ESCON), September 2006, Pultusk. 
Alberts, H., Martijn, C., \& deVries, N. K. (2006). Therelationship between expectancies and interpersonal differences in self-control performance. Paper presented at the 20th conference of the European Health Psychology Society (EHPS) August 2006, Warsaw.

Alberts, H., Maroofi, S., \& Martijn, C. (2005). De relatie tussen verwachtingen en interpersoonlijke verschillen in zelfcontrole. Paper presented at the 20th conference of the Association for Social Psychological Researchers (ASPO), december 2005, Maastricht.

Alberts, H., \& Martijn, C. (2005). Carry on or giving in: The role of (implicit) cognition in self-control. Research results presented at the 14th General Meeting of the EAESP, July 2005, Würzburg.

Alberts, H., Greb, J., \& Martijn, C. (2003). "You can do it.com!” Is zelfcontrole een kwestie van de juiste activatie? Paper presented at the 18th conference of the Association for Social Psychological Researchers (ASPO), December 2003, Maastricht.

Alberts, H., \& de Cremer, D. (2002). Positieve emotionele reacties en procedurele rechtvaardigheid: De effecten van inspraak en selectie van leiderschap als functie van de behoefte ergens bij te horen. Paper presented at the 17th conference of the Association for Social Psychological Researchers (ASPO), December 2002, Utrecht.

\section{Presentations on invitation}

Alberts, H., \& Martijn, C. (2006). The role of expectancies in the maintenance or loss of self-control. Research results presented at the 6th conference of Psychology and Health (P\&H), May 10, 2006, Kerkrade.

Alberts, H. (2005). Carry on or giving in: The role of (implicit) cognition in self-control. Oral presentation, Economic and Social Psychology division, September 9, Tilburg.

Alberts, H., \& Martijn, C. (2005). Carry on or giving in: The role of (implicit) cognition in the maintenance or loss of self-control. Research results presented at the 19th Conference of the European Health Psychology Society (EHPS), August 30, Galway, Ireland. 
Alberts, H., \& Martijn, C. (2005). Implicit cognition and self-control. Research results presented at the workshop "self-esteem and self-regulation", April 18, 2005, Nijmegen.

Alberts, H., \& Martijn, C. (2004). Carry on or giving in: The role of (implicit) cognition in the maintenance or loss of self-control. Research results presented at the fifth conference of Psychology and Health (P\&H), May 11 2004, Kerkrade.

Martijn, C., \& Alberts, H. (2003). Carrying on or giving in: Implicit cognition and self-control. Research results presented at the conference of Experimental Psychopathology (EPP), May 6, 2003, Nijmegen.

Alberts, H. (2003). Waar een wil is, is een weg? De mentale kosten van zelfcontrole. Oral presentation, Experimental Clinical Psychology division, February 21, Nijmegen. 
The "Kurt Lewin Institute Dissertation Series" started in 1997. Since 2005 the following dissertations have been published:

2005-1: Elsbeth Steenland: Stereotype Change: Effects of disconfirming information

2005-2: Annet de Lange: What about causality? Examining longitudinal relations between work characteristics and mental health

2005-3: Reint Jan Renes: Sustained volunteerism: Justification, motivation and management

2005-4: Aloyse Augusta Dinsbach: Socialization in the workplace: A focus on migrant groups

2005-5: Susanne Peters: The social psychology of being better off than others

2005-6: Winny Bakker: Emigration and well-being: The role of personality and cultural identity in acculturation

2005-7: Ruud Zaalberg: The expression of emotion in social situations. The mediating roles of display rules and social motives

2005-8: Catharine Evers: Sex differences in anger expressions. The shaping role of social appraisals

2005-9: Ed Sleebos: The Consequences of Perceived Intra-group Respect: The Effects of Differential Intra-group Respect on Behavior and Cognition

2005-10: Jeroen Stouten: Virtue Summons the Fury: Coordination Rules and Fairness in Social Dilemmas

2005-11: Irene de Pater: Doing Things Right or Doing the Right Thing: A New Perspective on the Gender Gap in Career Success

2005-12: Rob Nelissen: Guided by Reason, Struck by Emotion: Integrating Motivational \& Expectancy - Value Accounts of Behavior

2005-13: Emely de Vet: Testing the Transtheoretical Model: Validity and Applicability for Fruit Intake

2005-14: Floor Rink: Diversity and Small Group Decision Making: Towards a social identity framework for studying the effects of task-related differences on dyads and groups

2005-15: Sven Zebel: Negative Associations: The Role of Identification in Group-Based Guilt

2005-16: Eric Rietzschel: From quantity to quality: Cognitive, motivational and social aspects of creative idea generation and selection

2006-1: Maria Dijkstra: Workplace Conflict and Individual Well-Being

2006-2: Ruud Custers: On the underlying mechanisms of nonconscious goal pursuit

2006-3: Ellen Dreezens: The missing link: the relationship between values and attitudes 
2006-4: Jacquelien van Stekelenburg: Promoting or preventing social change. Instrumentality, identity, ideology and groupbased anger as motives of protest participation

2006-5: Huadong Yang: Siding in a conflict in China and in the Netherlands

2006-6: Tomas Ståhl: Determinants of Fairness-based and Favorability-based Reactions to Authorities' Decisions

2006-7: Astrid Homan: Harvesting the value in diversity: Examining the effects of diversity beliefs, cross-categorization, and superordinate identities on the functioning of diverse work groups

2006-8: Saskia Schwinghammer: The Self in Social Comparison

2006-9: Carmen Carmona Rodríguez: Inferior or Superior: Social Comparison in Dutch and Spanish Organizations

2006-10: Martijn van Zomeren: Social-psychological paths to protest: An integrative perspective

2007-1: Nils Jostmann: When the going gets tough... How action versus state orientation moderates the impact of situational demands on cognition, affect, and behavior

2007-2: Belle Derks: Social identity threat and performance motivation: The interplay between ingroup and outgroup domains

2007-3: Helma van den Berg: Feeling and Thinking in Attitudes

2007-4: Karin C.A. Bongers: You can't always get what you want! Consequences of success and failure to attain unconscious goals

2007-5: Lotte Scholten: Motivation matters: Motivated information processing in group and individual decision-making

2007-6: Debra Trampe: Social influence: Social comparison, construal, and persuasion processes

2007-7: Clemens Wenneker: Processes underlying biased language use

2007-8: Yaël de Liver: Ambivalence: on the how and when of attitudinal conflict

2007-9: Erik de Kwaadsteniet: Uncertainty in social dilemmas

2007-10: Hugo Alberts: Carrying on or giving in: Processes of self-control and ego depletion 